\title{
Closed quasi-Fuchsian surfaces in hyperbolic knot complements
}

\author{
JOSEPH D MASTERS \\ XINGRU ZHANG
}

\begin{abstract}
We show that every hyperbolic knot complement contains a closed quasi-Fuchsian surface.
\end{abstract}

$57 \mathrm{~N} 35 ; 57 \mathrm{M} 25$

\section{Introduction}

By a knot complement we mean, in this paper, the complement of a knot in a connected closed orientable 3-manifold (which is not necessarily $S^{3}$ ). A knot complement is said to be hyperbolic if it admits a complete hyperbolic metric of finite volume. By a surface we mean, in this paper, the complement of a finite (possibly empty) set of points in the interior of a compact, connected, orientable 2-manifold. By a surface in a 3-manifold $M$, we mean a continuous, proper map $f: S \rightarrow M$ from a surface $S$ into $M$. A surface $f: S \rightarrow M$ in a 3-manifold $M$ is said to be incompressible if $S$ is not a 2-sphere and the induced homomorphism $f^{*}: \pi_{1}(S, s) \rightarrow \pi_{1}(M, f(s))$ is injective for one (and thus for any) choice of base point $s$ in $S$. A surface $f: S \rightarrow M$ in a 3-manifold $M$ is said to be essential if it is incompressible and the map $f: S \rightarrow M$ cannot be homotoped into a boundary component or an end component of $M$.

Essential surfaces in hyperbolic knot complements can be divided into three mutually exclusive geometric types: quasi-Fuchsian surfaces, geometrically infinite surfaces and essential surfaces with accidental parabolics. Now we recall the relevant terminology. Let $\mathbb{H}^{3}$ denote the hyperbolic 3-space (always in the upper half space model) and let $S_{\infty}^{2}=\mathbb{C} \cup\{\infty\}$ denote the boundary at infinity, where $\mathbb{C}$ is the plane of complex numbers. Let $\overline{\mathbb{H}}^{3}=\mathbb{H}^{3} \cup S_{\infty}^{2}$ be the compactification of $\mathbb{H}^{3}$, which is topologically a compact 3-ball. The action of every element of the orientation preserving isometry group Isom ${ }^{+}\left(\mathbb{H}^{3}\right)$ extends to an action on $\overline{\mathbb{H}}^{3}$. For a discrete subgroup $\Gamma$ of Isom $^{+}\left(\mathbb{H}^{3}\right)$, let $\Lambda(\Gamma)$ denote the limit set of $\Gamma$ in $S_{\infty}^{2}$ and let $\Omega(\Gamma)=S_{\infty}^{2}-\Lambda(\Gamma)$ denote the regular set of $\Gamma$ in $S_{\infty}^{2}$. A discrete, torsion-free subgroup $\Gamma$ of $\operatorname{Isom}^{+}\left(\mathbb{H}^{3}\right)$ is called quasi-Fuchsian if its limit set $\Lambda(\Gamma)$ in $S_{\infty}^{2}$ is a Jordan circle and each of the 
two components of $\Omega(\Gamma)$ is invariant under the action of $\Gamma$. In the special case that the Jordan circle is a geometric circle, the subgroup is said to be Fuchsian.

If $M$ is a hyperbolic knot complement, then its fundamental group can be identified as a discrete torsion free subgroup $\Gamma$ of $\operatorname{Isom}^{+}\left(\mathbb{H}^{3}\right)$. A surface $f: S \rightarrow M$ in a hyperbolic knot manifold $M$ is said to be

(a) quasi-Fuchsian if it is essential and $f^{*}\left(\pi_{1}(S)\right)$ is a quasi-Fuchsian subgroup of $\Gamma \subset \operatorname{Isom}^{+}\left(\mathbb{H}^{3}\right)$; or

(b) geometrically infinite if it is essential and the limit set of $f^{*}\left(\pi_{1}(S)\right)$ is the entire $S_{\infty}^{2} ;$ or

(c) essential with accidental parabolics if it is essential and some non-peripheral element of $\pi_{1}(S)$ has a parabolic image in $f^{*}\left(\pi_{1}(S)\right) \subset \pi_{1}(M) \subset \operatorname{Isom}^{+}\left(\mathbb{H}^{3}\right)$.

A quasi-Fuchsian surface $f: S \rightarrow M$ is further called a Fuchsian or totally geodesic surface if the map lifts to a totally geodesic plane in $\mathbb{H}^{3}$ with respect to the universal covering $\mathbb{H}^{3} \rightarrow M$. In such case the image group $f^{*}\left(\pi_{1}(S)\right)$ is a Fuchsian subgroup of $\operatorname{Isom}^{+}\left(\mathbb{H}^{3}\right)$.

Work of Marden [13], Thurston [19] and Bonahon [4] implies that every essential surface falls into one of these categories. Another consequence of their work is that every geometrically infinite surface is homotopic to a virtual fiber. (It is still an open question whether every hyperbolic knot complement is virtually fibered.) In particular, if a closed essential surface in a hyperbolic knot complement has no accidental parabolics, then it is quasi-Fuchsian.

Examples of quasi-Fuchsian surfaces in hyperbolic knot complements have been scarce. It was shown by Cooper, Long and Reid [8] that every hyperbolic knot complement contains closed essential surfaces, but the surfaces constructed there (via Freedman tubing) always contain accidental parabolic elements. Similarly, the closed essential surfaces constructed in Oertel [16], Cooper-Long [6; 7] and Li [12], all contain accidental parabolics. It was shown by Menasco [15] that the complement of an alternating knot in $S^{3}$ contains no closed, embedded quasi-Fuchsian surface, a result which was extended in Adams [1]. On the positive side, there are well-known examples, such as the figure-eight knot complement, which contain closed, totally geodesic surfaces. Also, hyperbolic knot complements in $S^{3}$ which contain closed, embedded, quasi-Fuchsian surfaces are constructed in Adams and Reid [2]. In this paper we prove the following general existence theorem.

Theorem 1.1 Every hyperbolic knot complement contains a closed quasi-Fuchsian surface. 
A closed quasi-Fuchsian surface in a hyperbolic knot complement $M$ has the nice property that it remains essential in all but finitely many Dehn fillings of $M$ (see, for example, Wu [20, Theorem 5.3]). Theorem 1.1 thus has the following topological consequence:

Corollary 1.2 Suppose that $M$ is a hyperbolic knot complement. Then $M$ contains a closed essential surface which remains essential in all but finitely many Dehn fillings of $M$.

It was first shown in Cooper and Long [7], and later by a different method in Li [12], that for any hyperbolic knot complement $M$, all but finitely many Dehn fillings of $M$ contain a closed essential surface. What's new in Corollary 1.2 is that for every hyperbolic knot complement $M$, there is a single closed essential surface in $M$ which survives all but finitely many Dehn fillings of $M$.

We wish to thank the referee for many helpful comments.

\section{Outline of proof and plan of paper}

Let $M$ be a hyperbolic knot complement, and let $C$ be a geometric cusp of $M$. The complement of the interior of $C$ in $M$, which we denote by $M^{-}$, is a compact (connected and orientable) 3-manifold whose boundary is a torus. We call $M^{-}$the truncated knot complement. The idea is to construct a metrically complete convex hyperbolic 3-manifold $Y$ with the following properties:

(1) $Y$ has non-empty boundary;

(2) there is a local isometry $f$ from $Y$ into the knot complement $M$, and thus an injective homomorphism $f^{*}$ of $\pi_{1}(Y)$ into $\pi_{1}(M)$ (by Lemma 4.2);

(3) $Y$ has a single cusp, $C_{0}$, such that:

(i) the fundamental group of $C_{0}$ is a free abelian group of rank two which injects into the fundamental group of $Y$ under the inclusion map;

(ii) the image of $\pi_{1}\left(C_{0}\right)$ under the map $f^{*}$ is a finite index subgroup of $\pi_{1}(C)$;

(iii) every Dehn filling of $Y$ along the cusp $C_{0}$ results a compact 3-manifold which is $\partial$-irreducible.

Restricting $f$ to any boundary component of $Y$ gives a closed surface in $M$, and the above properties imply that the surface is quasi-Fuchsian. The proof of this implication is given at the end of Section 13. 
To construct such a manifold $Y$, we start with a pair of (non-compact) embedded, quasi-Fuchsian surfaces $S_{i}, i=1,2$, in $M$ such that $S_{i}^{-}=S_{i} \cap M^{-}, i=1,2$, are properly embedded essential surfaces with different boundary slopes on $\partial M^{-}$. The existence of such a pair of surfaces follows from work of Culler and Shalen [9]. Let $n_{i}$ be the number of components of $\partial S_{i}^{-}$and let $\Delta$ be the geometric intersection number between a component of $\partial S_{1}^{-}$and a component of $\partial S_{2}^{-}$. The fundamental group of $S_{i}$ can be naturally identified with a fixed quasi-Fuchsian subgroup $\Gamma_{i}$ of $\Gamma=\pi_{1}(M)$. The limit set $\Lambda_{i}$ of $\Gamma_{i}$ is a Jordan circle in $S_{\infty}^{2}$. Let $H_{i}$ be the convex hull of $\Lambda_{i}$ in $\mathbb{H}^{3}$, and let $X_{i}$ be the $\epsilon$-collared neighborhood of $H_{i}$ in $\mathbb{H}^{3}$ for some fixed number $\epsilon>0$. Then each of $H_{i}$ and $X_{i}$ is a convex 3-submanifold of $\mathbb{H}^{3}$ invariant under the action of $\Gamma_{i}$. Let $Y_{i}=X_{i} / \Gamma_{i}$. Then $Y_{i}$ is a metrically complete convex hyperbolic 3-manifold with a local isometry $f_{i}$ into $M$. Topologically $Y_{i}$ is a product $I$-bundle over $S_{i}$, ie $Y_{i}=S_{i} \times I$. We have the corresponding truncated $I$-bundle $Y_{i}^{-}=S_{i}^{-} \times I$. The "cusp region" of $Y_{i}$ has a standard shape if the geometric cusp $C$ of $M$ is chosen small enough. In particular, the parabolic boundary $\partial_{p} Y_{i}^{-} \equiv \partial S_{i}^{-} \times I$, is a set of $n_{i}$ standard Euclidean annuli.

To illustrate how $Y$ is constructed, let us make some simplifying assumptions. Suppose that each $S_{i}$ is totally geodesic, that $Y_{i}^{-}$is an $\epsilon$-neighborhood of $S_{i}^{-}$, embedded in $M$, and that $S_{1}^{-} \cap S_{2}^{-}$has a large collar neighborhood in both $S_{1}^{-}$and $S_{2}^{-}$. In this case, we construct $Y$ as an embedded sub-manifold of $M$. Consider $Y_{1}^{-} \cup Y_{2}^{-}$, which is a sub-manifold of $M^{-}$. The boundary of this submanifold is convex, except along the "corners" ( $\left.\partial Y_{1}^{-} \cap \partial Y_{2}^{-}\right)$, and along the truncated cusp. Since we have assumed that the components of $S_{1}^{-} \cap S_{2}^{-}$are well spread out, there is enough room to smooth out the corners, as illustrated in Figure 1 (which shows the part of the smoothing near $\partial_{p} Y_{1}^{-} \cup \partial_{p} Y_{2}^{-} \subset \partial M^{-}$). We thus obtain a truncated sub-manifold, $Y^{-} \subset M^{-}$, whose frontier is convex. The complement of int $Y^{-}$in $\partial M^{-}$consists of a finite number of disks, and the convex hull of each disk is a compact subset of the cusp $C$. We scoop out each of these convex sets from $C$ to form a new cusp $C_{0}$. The manifold $Y$ is the union of $Y^{-}$and $C_{0}$.

In general, we cannot hope for the manifolds $Y_{i}^{-}$to be embedded in $M^{-}$, and so we must construct $Y$ in a more abstract way. We wish to identify $Y_{1}^{-}$and $Y_{2}^{-}$ along certain isometric embedded submanifolds $K_{i}^{-} \subset Y_{i}^{-}$, which correspond to "intersection" components of $Y_{1}^{-}$and $Y_{2}^{-}$. We then wish to smooth out the corners to form a hyperbolic 3-manifold $Y^{-}$which is locally convex everywhere except on its "parabolic boundary" $\partial_{p} Y^{-}$. Then we wish to attach a cusp $C_{0}$ along $\partial_{p} Y^{-}$to form the required manifold $Y$.

The gluing and smoothing operations are well-known in the totally geodesic case, but to make them work for quasi-Fuchsian surfaces is more difficult. Furthermore, the gluing 


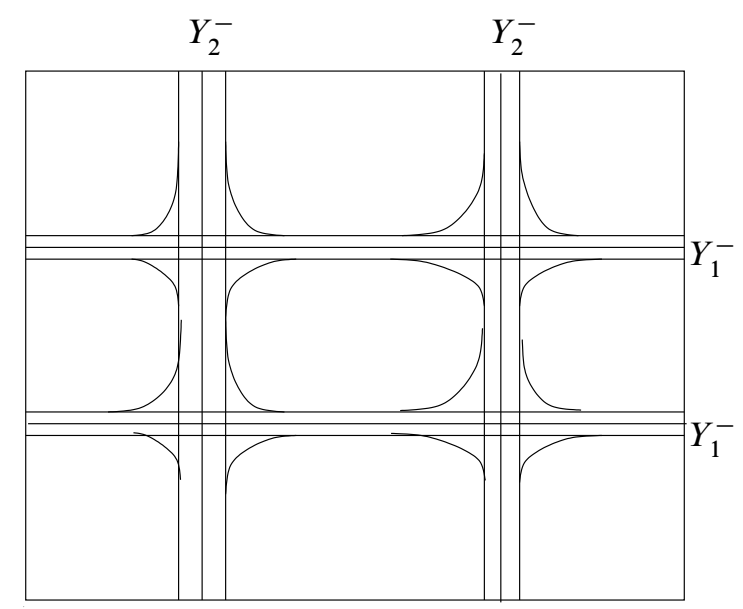

Figure 1

can only be performed on manifolds with sufficient "room". Thus it may be necessary to replace the given manifolds $Y_{i}^{-}$with suitable finite covers $\breve{Y}_{i}^{-}$. We construct such covers by proving that free groups satisfy a strengthened form of the LERF property.

In Section 4, we collect some general facts about hyperbolic geometry. Of particular importance is a general fact about convex hulls in hyperbolic space (Proposition 4.5), which is essential for our gluing constructions. In Section 5, we give some general facts about cusped, quasi-Fuchsian surfaces, and their convex cores.

In Section 6, we construct the "gluing manifolds" $K_{1}^{-}$and $K_{2}^{-}$. In the case where each $Y_{i}^{-}$is embedded in $M^{-}$, then $K_{i}^{-} \subset Y_{i}^{-}$is just the intersection $Y_{1}^{-} \cap Y_{2}^{-}$. In general, the fundamental group of each component of $K_{i}^{-}$is identified with the intersection of some conjugate of $\Gamma_{1}=f^{*} \pi_{1} S_{1}^{-}$and some conjugate of $\Gamma_{2}=f^{*} \pi_{1} S_{2}^{-}$, and there is an immersion $g_{i}: K_{i}^{-} \rightarrow Y_{i}^{-}$.

The gluing must occur along embedded sub-manifolds of $Y_{i}^{-}$, and so we must lift $g_{i}$ to an embedding. For this purpose, it will be useful to isometrically embed $K_{i}^{-}$into a connected hyperbolic manifold $J_{i}^{-}$, whose boundary is convex, outside of a compact set of parabolic regions, and which has a local isometry map (still denoted $g_{i}$ ) into $Y_{i}^{-}$. The construction of $J_{i}^{-}$is contained in Section 7.

We also wish to control how the parabolic boundary of $J_{i}^{-}$is located in $\partial Y_{i}^{-}$under the local isometry $g_{i}: J_{i}^{-} \rightarrow Y_{i}^{-}$, and for this purpose we embed $J_{i}^{-}$isometrically into a certain compact, convex hyperbolic manifold $C_{n}\left(J_{i}^{-}\right)$. We also extend $g_{i}$ to a local isometry $g_{i}: C_{n}\left(J_{i}^{-}\right) \rightarrow Y_{i}$. The construction of $C_{n}\left(J_{i}^{-}\right)$is contained in Section 8 . 
Free groups are LERF, and so, using standard arguments, it is possible to find a finite cover $\breve{Y}_{i}$ of $Y_{i}$ such that the map $g_{i}: C_{n}\left(J_{i}^{-}\right) \rightarrow Y_{i}$ lifts to an embedding. However, for our construction, we require the corresponding truncated cover $\breve{Y}_{i}^{-}=\breve{S}_{i}^{-} \times I$ to have the same number of parabolic boundary components as that of $Y_{i}^{-}$. Thus we must show that free groups satisfy a strengthened version of the LERF Property. This is done in Section 9. The proof of this stronger LERF property requires much more work than the classical LERF property, and may be of independent group theoretic interest. The proof applies Stallings' graph-folding techniques.

Finally we need to impose one more technical condition on the covers $\breve{Y}_{i}$. We require that, after the gluing, $\partial_{p} \breve{Y}_{1}^{-} \cup \partial_{p} \breve{Y}_{2}^{-}$is isometric to an embedded grid in a certain finite cover $\breve{T}$ of the Euclidean torus $\partial C$. The exterior of the grid should be a set of Euclidean parallelograms with long sides. This requires a further strengthening of the LERF property for free groups, which is carried out in Section 11.

With this property achieved, we can cap off $Y^{-}$with a hollowed solid cusp $C_{0}$ along $\partial_{p} Y^{-}$to get a metrically complete convex hyperbolic 3-manifold $Y$, with non-empty boundary, with a single cusp, and with a local isometry into $M$. Thus $Y$ already has the required properties (1) and (2) given above. To show $Y$ has the property (3), we show that any Dehn filling $Y(\alpha)$ of $Y$ with slope $\alpha$ can be decomposed, in a specified way, into handlebody and $I$-bundle pieces. We call such manifold an HS-manifold. In Section 12 we show that if an HS-manifold satisfies certain conditions then its boundary is incompressible.

Our last step is to show that the HS-manifold structure of $Y(\alpha)$ satisfies these conditions for incompressibility. The final assembly of $Y$, and the proof that $Y$ has all the required properties, are given in Section 13.

We remark that Baker and Cooper have recently obtained results on gluing convex hyperbolic manifolds [3], which overlap with some of our gluing results, for example in Section 4.

\section{Conventions}

In this paper, all manifolds shall be assumed orientable by default. Any 0 -codimension submanifold of an oriented manifold is given the induced orientation in the obvious way. If $\widetilde{W}$ is a covering space of an oriented manifold $W$, then the induced orientation for $\widetilde{W}$ is the one which makes the covering map orientation preserving. If $W$ is an oriented $n$-manifold ( $n \geq 1$ ) with boundary, then its boundary $\partial W$ is given the induced orientation according the following rule: at each point of $\partial W$, the induced orientation 
of $\partial W$ followed by an inward pointing tangent vector of $W$ gives the orientation of $W$ at that point.

Suppose that $U_{i}$ is a submanifold of a manifold $V_{i}, i=1,2$, then a map of pairs $f:\left(V_{1}, U_{1}\right) \rightarrow\left(V_{2}, U_{2}\right)$ is called proper if the pre-image of any compact set is compact, and if $f\left(U_{1}\right) \subset U_{2}$.

If $V$ is a hyperbolic 3-manifold, then for any submanifold $U$ of $V$ (in particular $\partial V$ ), each component of $U$ is considered as a metric space with the induced path metric. If $\tilde{V}$ is a connected covering space of $V$, then $\widetilde{V}$ is given the induced metric so that the covering map from $\tilde{V}$ to $V$ is a local isometry.

If $V$ is a metric space and $U$ is a subset of $V$, then $V-U$ denotes the complement of $U$ in $V$, and $V \backslash U$ denotes the set obtained by first taking the topological closure of individual components of $V-U$ in $V$ and then taking the disjoint union of these closures.

We say a connected subspace $U$ of a space $V$ carries the fundamental group of $V$ if the inclusion $U \subset V$ induces a surjective homomorphism on the fundamental groups.

\section{Some properties of convex hyperbolic 3-manifolds}

For standard definitions and facts about hyperbolic manifolds (possibly with boundary), the limit set, the convex hull, the developing map, the holonomy representation, etc, we take Canary-Epstein-Green [5], Epstein-Marden [10] and Ratcliffe [17] as references.

For any subset $W$ of $\mathbb{H}^{3}$, the limit set of $W$ in $S_{\infty}^{2}$, denoted $\Lambda(W)$, is the set of intersection points (possibly empty) between the closure of $W$ in $\overline{\mathbb{H}}^{3}$ and $S_{\infty}^{2}$.

Let $V$ be an orientable, metrically complete, convex (thus connected) hyperbolic 3manifold (possibly with boundary), with base point $v_{0} \in V$. Then its universal cover $\tilde{V}$ is also a metrically complete, convex, hyperbolic 3 -manifold, and the developing map $D: \widetilde{V} \rightarrow \mathbb{H}^{3}$ is an isometry of $\widetilde{V}$ onto its image [5, Proposition 1.4.2]. It follows that the holonomy representation $\rho$ of $\pi_{1}\left(V, v_{0}\right)$ into $P S L_{2}(\mathbb{C})$ is a discrete and faithful representation with no nontrivial elliptic elements in the image. The image group $\Gamma=\rho\left(\pi_{1}\left(V, v_{0}\right)\right)$ acts on $D(\tilde{V})$ as a covering transformation group. So we may consider $\widetilde{V}$ as a submanifold of $\mathbb{H}^{3}$ and consider $V$ as the quotient space of $\widetilde{V}$ under the action of $\Gamma$. Let $p: \widetilde{V} \rightarrow V$ be the quotient map, which is a universal covering map, and let $\widetilde{v}_{0} \in \tilde{V}$ be a fixed point in $p^{-1}\left(v_{0}\right)$. Then the fundamental group $\pi_{1}\left(V, v_{0}\right)$ can be identified with $\Gamma$ in the following way. Let $\alpha:([0,1], \partial[0,1]) \rightarrow\left(V, v_{0}\right)$ be a loop in $V$, based at $v_{0}$, representing a nontrivial element $\alpha_{*}$ of $\pi_{1}\left(V, v_{0}\right)$, and let 
$\tilde{\alpha}:([0,1], 0) \rightarrow\left(\tilde{V}, \widetilde{v}_{0}\right)$ be the unique lift of $\alpha$ based at the point $\widetilde{v}_{0}$ with respect to the covering map $p:\left(\widetilde{V}, \widetilde{v}_{0}\right) \rightarrow\left(V, v_{0}\right)$. Then the element of $\Gamma$ corresponding to $\alpha_{*}$ is the one which maps $\widetilde{v}_{0}$ to $\widetilde{a}(1)$.

A nontrivial element $\gamma$ of $\pi_{1}\left(V, v_{0}\right)$ is said to be hyperbolic or parabolic if $\rho(\gamma) \in \Gamma$ is hyperbolic or parabolic, respectively, in the usual sense; ie $\gamma$ has exactly two fixed points or one fixed point, respectively, in $\overline{\mathbb{H}}^{3}$. This definition is independent of the choices for base points.

Let $V$ be a hyperbolic 3-manifold and $v_{0}$ a point in $V$. We define a geodesic loop in $V$ based at $v_{0}$ to be a loop $\alpha:([0,1], \partial[0,1]) \rightarrow\left(V, v_{0}\right)$, which is geodesic when restricted to $(0,1)$. Throughout this paper, a geodesic is always assumed to be non-constant.

Lemma 4.1 Let $V$ be an orientable, metrically complete, convex, hyperbolic 3manifold (possibly with boundary), and $v_{0} \in V$ a base point. Then every nontrivial element in $\pi_{1}\left(V, v_{0}\right)$ is represented uniquely by a geodesic loop in $V$ based at $v_{0}$.

Proof We identify $\tilde{V}$, the universal cover of $V$, as a metrically complete, convex submanifold of $\mathbb{H}^{3}$, and let $p: \tilde{V} \rightarrow V$ be the covering map. Fix a point $\widetilde{v}_{0}$ in $p^{-1}\left(v_{0}\right)$ as the base point of $\tilde{V}$. For a given nontrivial element $\gamma \in \pi_{1}\left(V, v_{0}\right)$, let $\alpha:[0,1] \rightarrow V$ be a loop in $V$ based at $v_{0}$ (ie $\alpha(0)=\alpha(1)=v_{0}$ ) representing $\gamma$. Let $\tilde{\alpha}:[0,1] \rightarrow \widetilde{V}$ be the unique lift of $\alpha$ with $\widetilde{\alpha}(0)=\widetilde{v}_{0}$. Since $\alpha$ represents a nontrivial element of $\pi_{1}\left(V, v_{0}\right), \widetilde{\alpha}(0) \neq \widetilde{\alpha}(1)$. Let $\widetilde{\sigma}:[0,1] \rightarrow \mathbb{H}^{3}$ be the unique geodesic segment with $\tilde{\sigma}(0)=\tilde{\alpha}(0)=\widetilde{v}_{0}$ and $\tilde{\sigma}(1)=\tilde{\alpha}(1)$. Since $\tilde{V}$ is convex, the geodesic path $\tilde{\sigma}$ is contained in $\tilde{V}$. Thus the map $\sigma=p \circ \tilde{\sigma}:[0,1] \rightarrow V$ gives a geodesic loop in $V$ based at $v_{0}$. By convexity, the convex hull of the set $\widetilde{\alpha}([0,1]) \cup \widetilde{\sigma}([0,1])$ is contained in $\tilde{V}$, and this hull contains a homotopy between $\tilde{\alpha}$ and $\widetilde{\sigma}$ with their endpoints fixed. Under the covering map $p$, the homotopy descends to a homotopy in $V$ between the loop $\alpha$ and the geodesic loop $\sigma$ fixing the base point $v_{0}$. Hence $\sigma$ is also a representative loop of the element $\gamma$. The uniqueness of such a based geodesic loop is clear from the argument.

Lemma 4.2 Suppose that $f: U \rightarrow V$ is a local isometry between two 3-manifolds $U$ and $V$ which are orientable, metrically complete, convex and hyperbolic. Then $f^{*}: \pi_{1}\left(U, u_{0}\right) \rightarrow \pi_{1}\left(U, f\left(u_{0}\right)\right)$ is injective for any choice of the base point $u_{0}$ in $U$. If in addition $U$ is compact, then $f^{*}\left(\pi_{1}\left(U, u_{0}\right)\right)$ contains no parabolic elements of $\pi_{1}\left(V, f\left(u_{0}\right)\right)$.

Proof Let $v_{0}=f\left(u_{0}\right)$, let $p: \tilde{V} \rightarrow V$ be the universal covering map, where $\tilde{V}$ is identified as a submanifold of $\mathbb{H}^{3}$, and let $\widetilde{v}_{0}$ be a fixed point in $p^{-1}\left(v_{0}\right)$. To prove 
the first assertion, let $\gamma$ be a nontrivial element of $\pi_{1}\left(U, u_{0}\right)$. By Lemma 4.1, $\gamma$ is represented by a geodesic loop $\sigma$ in $U$ based at $u_{0}$. Since $f$ is a local isometry, $f \circ \sigma$ is a geodesic loop in $V$ based at $v_{0}$. If $f^{*}(\gamma)$ is the trivial element of $\pi_{1}\left(V, v_{0}\right)$, then $f \circ \sigma$ lifts to a geodesic loop in $\widetilde{V}$ based at $\widetilde{v}_{0}$. But obviously $\mathbb{H}^{3}$ contains no based geodesic loops. Hence $f^{*}(\gamma)$ is nontrivial in $\pi_{1}\left(V, v_{0}\right)$, and thus $f^{*}$ is injective.

Now suppose in addition that $U$ is compact. Let $H=f^{*}\left(\pi_{1}\left(U, u_{0}\right)\right)$. Let $\bar{V}=\tilde{V} / H$, and let $\bar{q}:\left(\tilde{V}, \widetilde{v}_{0}\right) \rightarrow\left(\bar{V}, \bar{q}\left(\widetilde{v}_{0}\right)\right), \bar{p}:\left(\bar{V}, \bar{q}\left(\widetilde{v}_{0}\right)\right) \rightarrow\left(V, v_{0}\right)$ be the covering maps. Since $\bar{p}^{*}\left(\pi_{1}\left(\bar{V}, \bar{q}\left(\widetilde{v}_{0}\right)\right)\right)=f^{*}\left(\pi_{1}\left(U, u_{0}\right)\right)=H$, the map $f:\left(U, u_{0}\right) \rightarrow\left(V, v_{0}\right)$ lifts to a map $\bar{f}:\left(U, u_{0}\right) \rightarrow\left(\bar{V}, \bar{q}\left(\widetilde{v}_{0}\right)\right)$. Since $\bar{p} \circ \bar{f}=f$ and since $\bar{p}$ and $f$ are local isometries, $\bar{f}$ is a local isometry.

Let $p^{\prime}:\left(\tilde{U}, \tilde{u}_{0}\right) \rightarrow\left(U, u_{0}\right)$ be the universal covering map. Then the map $\bar{f} \circ p^{\prime}$ lifts to a map $\tilde{f}:\left(\widetilde{U}, \tilde{u}_{0}\right) \rightarrow\left(\widetilde{V}, \widetilde{v}_{0}\right)$. Since $\bar{f} \circ p^{\prime}=\bar{q} \circ \tilde{f}$ and since $\bar{q}, p^{\prime}, \bar{f}$ are all local isometries, $\tilde{f}$ is also a local isometry. Hence $\tilde{f}$ sends geodesic arcs to geodesic arcs. Since $\tilde{U}$ is convex and since $\tilde{V}$ is a simply connected submanifold of $\mathbb{H}^{3}, \tilde{f}$ must be an embedding. Since the map $\tilde{f}$ is equivariant and the map $f^{*}$ is an isomorphism, from the commutative diagram:

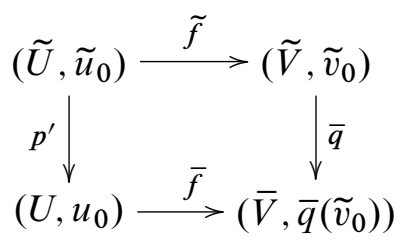

we see that $\bar{f}$ is an embedding. Hence $\tilde{f}(\tilde{U})$ is a convex submanifold of $\tilde{V}$ covering the compact submanifold $\bar{f}(U)$ of $\bar{V}$. In fact, $\tilde{f}(\tilde{U}) / H=\bar{f}(U)$.

If $H=f^{*}\left(\pi_{1}\left(U, u_{0}\right)\right)$ contains parabolic elements, then a standard hyperbolic geometry argument shows that $\bar{f}(U)$ contains a non-compact cusp end. In fact if $H_{0}$ is a nontrivial maximal parabolic subgroup of $H$ and if $a \in S_{\infty}^{2}$ is the point fixed by $H_{0}$, then there is a horoball $B_{a}$ of $\mathbb{H}^{3}$, based at $a$, such that $\left(B_{a} \cap \tilde{f}(\tilde{U})\right) / H_{0}$ properly embeds into $\bar{f}(U)$ as a non-compact end. This is a contradiction, since $\bar{f}(U)$ is compact.

Every metrically complete, convex subset of $\mathbb{H}^{3}$ is a manifold (Epstein-Marden [10, Theorem 1.4.3]). Obviously the intersection of two metrically complete, convex submanifolds of a metrically complete, convex 3-manifold is a metrically complete, convex submanifold (when non-empty). Every metrically complete, convex 3-submanifold $U$ of a simply connected, metrically complete, convex, hyperbolic 3 -manifold $V$ is simply connected (which follows from Lemma 4.2). A metrically complete, hyperbolic 
3-manifold (possibly with boundary) is convex or strictly convex if and only if it is everywhere locally convex or locally strictly convex, respectively (Canary-EpsteinGreen [5, Corollary 1.3.7]). These facts will be often used in this paper.

Let $V$ be a connected metric space and $U$ a subspace of $V$ (possibly disconnected). By an $r$-neighborhood of $U$ in $V$, denoted $N_{(r, V)}(U)$, we mean the set of points in $V$ whose distance from $U$ is less than or equal to $r$. Note that the topology of $N_{(r, V)}(U)$ may be different from that of $U$. An $r$-neighborhood $N_{(r, V)}(U)$ is further called an $r$-collared neighborhood of $U$ in $V$ if, under a universal covering map $p: \widetilde{V} \rightarrow V$, the components of $p^{-1}(U)$ are more than distance $2 r$ apart from each other. When the ambient space $V$ is clear, we simply write $N_{r}(U)$ for $N_{(r, V)}(U)$. The following lemma follows directly from the definition.

Lemma 4.3 If $V$ is a simply connected hyperbolic manifold and $U$ a connected submanifold of $V$, then for any $r>0, N_{(r, V)}(U)$ is an $r$-collared neighborhood of $U$ in $V$.

We also need to define " $r$-collared neighborhood" in relative version, as follows. Let $V$ be a connected, hyperbolic manifold with boundary and $F$ a submanifold of $\partial V$ (possibly with infinitely many components). Suppose that $U$ is a submanifold of $V$ and let $E=\partial U \cap F$ (which possibly has infinitely many components). If there is an $r$-collared neighborhood $N_{(r, V)}(U)$ of $U$ in $V$ such that for each component $F_{i}$ of $F, N_{(r, V)}(U) \cap F_{i}$ is an $r$-collared neighborhood of $E \cap F_{i}$ in $F_{i}$ (where $F_{i}$ is given the induced metric as a submanifold of $V)$, then we say that the pair $(U, E)$ has an $r$-collared neighborhood in the pair $(V, F)$. Again directly from the definition we have the following lemma.

Lemma 4.4 Suppose that $V$ is a simply connected hyperbolic manifold and $F$ a submanifold of $\partial V$ such that each component of $F$ is simply connected. Suppose that $U$ is a connected submanifold of $V$ and suppose that for each component $F_{i}$ of $F$, $F_{i} \cap \partial U$ is a connected submanifold of $F_{i}$. Then for any $r>0$, the pair $(U, \partial U \cap F)$ has an $r$-collared neighborhood in the pair $(V, F)$.

For a metrically complete, convex submanifold $V \subset \mathbb{H}^{3}$ and a point $v$ in the frontier of $V$ in $\mathbb{H}^{3}$, we use $P_{(v, V)}$ to denote a support plane for $V$ at the point $v$, ie $P_{(v, V)}$ is a hyperbolic plane in $\mathbb{H}^{3}$ such that $V$ lies on one side of the plane and such that $V \cap P_{(v, V)}$ contains the point $v$. A supporting plane always exists [10, Lemma 1.4.5]. Let $\epsilon$ be a fixed positive number. For a metrically complete, convex submanifold $V$ in $\mathbb{H}^{3}$, the $\epsilon$-collared neighborhood of $V$ in $\mathbb{H}^{3}, N_{\epsilon}(V)$, is a metrically complete and strictly convex [10, Lemma 1.4.7] 3-dimensional submanifold of $\mathbb{H}^{3}$, with $C^{1}$ 
boundary [10, Lemma 1.3.6]. Note that the supporting plane of $N_{\epsilon}(V)$ at a point $x$ in the frontier of $N_{\epsilon}(V)$ (which is $\partial N_{\epsilon}(V)$ in this case) is unique, and intersects $N_{\epsilon}(V)$ only at the point $x$, due to the strict convexity of $N_{\epsilon}(V)$.

The following proposition will play a key role.

Proposition 4.5 For any given $\epsilon>0$, there is a number $R=R(\epsilon)>0$ such that the following holds. If $V$ and $V^{\prime}$ are metrically complete, convex submanifolds of $\mathbb{H}^{3}$ such that $N_{\epsilon}(V)$ and $V^{\prime}$ have non-empty intersection, and if $x$ is a point in $\partial N_{\epsilon}(V)$ such that $d\left(x, N_{\epsilon}(V) \cap V^{\prime}\right)>R$, then $P_{\left(x, N_{\epsilon}(V)\right)} \cap V^{\prime}=\varnothing$. In particular if we take the convex hull of the union of $N_{\epsilon}(V)$ and $N_{\epsilon}\left(V^{\prime}\right)$ then all the added points are contained in an $R$-collared neighborhood of $N_{\epsilon}(V) \cap N_{\epsilon}\left(V^{\prime}\right)$.

Proof Suppose otherwise that such $R$ does not exist. Let $x \in \partial\left(N_{\epsilon}(V)\right)$ be a point very far from $N_{\epsilon}(V) \cap V^{\prime}$, let $A$ be a geodesic segment, tangent to $N_{\epsilon}(V)$ at $x$, contained in the unique supporting plane $P_{\left(x, N_{\epsilon}(V)\right)}$, and suppose that $A \cap V^{\prime}$ contains a point $x^{\prime}$.

If $\partial\left(N_{\epsilon}(V)\right) \cap V^{\prime}=\varnothing$, then $V^{\prime} \subset$ int $N_{\epsilon}(V)$, and so $P_{\left(x, N_{\epsilon}(V)\right)} \cap V^{\prime}=\varnothing$. Thus, we may assume that $\partial\left(N_{\epsilon}(V)\right) \cap V^{\prime}$ contains a point $w$. Since every component of $\partial N_{\epsilon}(V)$ separates $\mathbb{H}^{3}$, we may assume that $w$ and $x$ are in the same component of $\partial N_{\epsilon}(V)$. Let $B$ be a geodesic segment from $x^{\prime}$ to $w$, let $C$ be a geodesic segment from $w$ to $x$, and let $P_{0}$ be the unique geodesic plane containing the (distinct) points $x, x^{\prime}$ and $w$. See Figure 2 .

Let $x_{1}$ and $w_{1}$ be the nearest points in $V$ to $x$ and $w$ respectively, let $C_{1}$ be a geodesic segment from $x_{1}$ to $w_{1}$, and let $E$ be the geodesic rectangle in $\mathbb{H}^{3}$ with vertices $x, x_{1}, w_{1}$ and $w$. Since $E$ bounds a surface of area less than $2 \pi$, then if $C$ and $C_{1}$ are long enough, most of the arc $C$ is very close to $C_{1}$; for example, we may assume that:

$$
\text { Length }\left(C \cap N_{.01 \epsilon}\left(C_{1}\right)\right) \geq .99 \text { Length }(C)
$$

Now let $D$ be the segment of the curve $P_{0} \cap \partial\left(N_{\epsilon}(V)\right)$ which runs from $x$ to $w$. Since $D \subset \partial N_{\epsilon} V$, and $C_{1} \subset V$, then:

$$
N_{\epsilon} C_{1} \cap D=\varnothing
$$

By (4-1) and (4-2), we have:

$$
\text { Length }\left(C-N_{\epsilon} D\right) \geq .99 \text { Length }(C)
$$




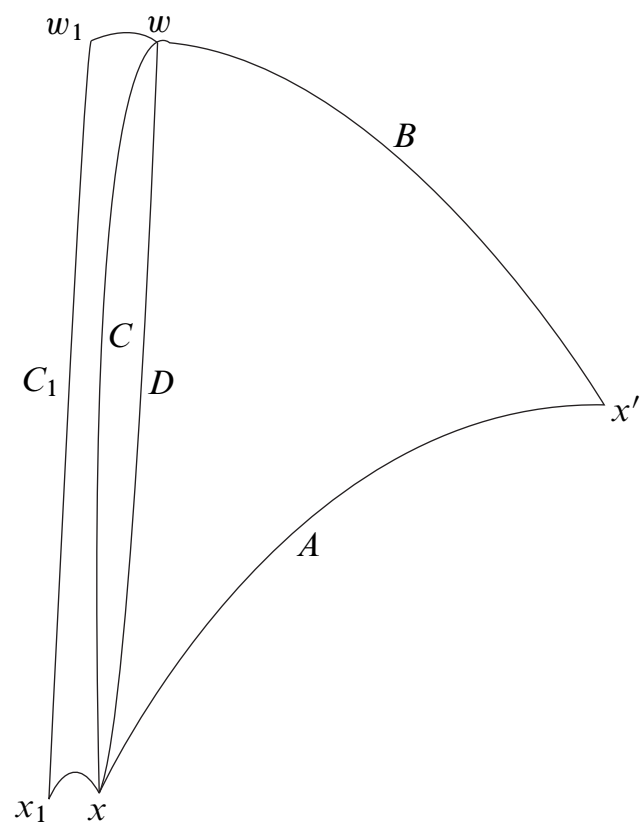

Figure 2: If $C$ is long, the area between $C$ and $D$ becomes large.

By (4-3) and a simple integration, the area in $P_{0}$ bounded by $C$ and $D$ is at least .99 Length $(C) * .99 \epsilon$. But since this region is contained in the triangle region $\mathrm{ABC}$, its area must be less than $\pi$, which is a contradiction if $C$ is long enough.

In a similar vein, we have:

Proposition 4.6 Suppose that $X$ is a convex submanifold of $\mathbb{H}^{3}$, that $V_{1}, \ldots, V_{n}$ are convex subsets of $X$, and that $N_{(\epsilon, X)}\left(V_{1}\right), \ldots, N_{(\epsilon, X)}\left(V_{n}\right)$ are all disjoint, for some $\epsilon>0$. Then $\operatorname{Hull}\left(N_{(\epsilon, X)}\left(V_{1}\right) \cup \ldots \cup N_{(\epsilon, X)}\left(V_{n}\right)\right) \backslash\left(N_{(\epsilon, X)}\left(V_{1}\right) \cup \ldots \cup N_{(\epsilon, X)}\left(V_{n}\right)\right)$ is compact.

Proof We first note that for any convex subset $V$ of $X, N_{(\epsilon, X)}(V)=N_{\epsilon}(V) \cap X$ and that $\partial N_{(\epsilon, X)}(V) \cap$ int $X=\partial N_{\epsilon}(V) \cap$ int $X$.

For every $x$ in $\partial N_{(\epsilon, X)}\left(V_{i}\right) \cap$ int $X$, there is a geodesic plane $P_{x}$, such that $P_{x} \cap$ $N_{(\epsilon, X)}\left(V_{i}\right)=\{x\}$. Let $\epsilon^{\prime}>0$ be a number such that $N_{(\epsilon, X)}\left(V_{i}\right) \cap N_{\left(\epsilon^{\prime}, X\right)}\left(V_{j}\right) \neq \varnothing$ for all $1 \leq i, j \leq n$. Since $N_{(\epsilon, X)}\left(V_{i}\right)$ and $N_{(\epsilon, X)}\left(V_{j}\right)$ are disjoint, the limit set of $N_{(\epsilon, X)}\left(V_{i}\right)$ is disjoint from the limit set of $N_{(\epsilon, X)}\left(V_{j}\right)$. Thus $N_{(\epsilon, X)}\left(V_{i}\right) \cap N_{\left(\epsilon^{\prime}, X\right)}\left(V_{j}\right)$ is compact in $\mathbb{H}^{3}$. The proof of Proposition 4.5 shows that there is a compact subset 
$B_{i} \subset N_{(\epsilon, X)}\left(V_{i}\right)$ such that for all $x \in\left(\partial N_{(\epsilon, X)}\left(V_{i}\right) \backslash B_{i}\right) \cap \operatorname{int}(X)$, we have $P_{x} \cap$ $N_{\epsilon^{\prime}}\left(V_{j}\right)=\varnothing$ for each $j \neq i$. It follows that $\operatorname{Hull}\left(N_{(\epsilon, X)}\left(V_{1}\right) \cup \ldots \cup N_{(\epsilon, X)}\left(V_{n}\right)\right) \backslash$ $\left(N_{(\epsilon, X)}\left(V_{1}\right) \cup \ldots \cup N_{(\epsilon, X)}\left(V_{n}\right)\right)$ has no limit points in $S_{\infty}^{2}$ and thus is compact.

Let $\Gamma$ be a group, $H \subset \Gamma$ a subgroup, and $\gamma$ an element in $\Gamma-H$. We say that $H$ is separable from $\gamma$ in $\Gamma$ if there exists a finite index subgroup $G$ of $\Gamma$ such that $G$ contains $H$ but does not contain $\gamma$. If $H$ is separable from every element in $\Gamma-H$, then $H$ is said to be separable in $\Gamma$. It is easy to see that if $H$ is separable in $\Gamma$, then given any finite set of elements $y_{1}, \ldots, y_{a}$ in $\Gamma-H$, there is a finite index subgroup $G$ of $\Gamma$ such that $G$ contains $H$ but does not contain any of $y_{1}, \ldots, y_{a}$.

Proposition 4.7 Let $U$ be a compact, convex, hyperbolic 3-manifold, let $V$ be a metrically complete, convex, hyperbolic 3-manifold and let $f:\left(U, u_{0}\right) \rightarrow\left(V, v_{0}\right)$ be a local isometry. Then there is a finite (possibly empty) set of elements $y_{1}, \ldots, y_{a}$ in $\pi_{1}\left(V, v_{0}\right)-f^{*}\left(\pi_{1}\left(U, u_{0}\right)\right)$ with the following property: if $G \subset \Gamma=\pi_{1}\left(V, v_{0}\right)$ is a finite index subgroup which separates $H=f^{*}\left(\pi_{1}\left(U, u_{0}\right)\right)$ from $y_{1}, \ldots, y_{a}$, and if $\bar{V}$ is the finite cover of $V$ corresponding to $G$, then the map $f:\left(U, u_{0}\right) \rightarrow\left(V, v_{0}\right)$ lifts to an embedding $\breve{f}: U \rightarrow \bar{V}$.

Proof Let $p:\left(\tilde{V}, \tilde{v}_{0}\right) \rightarrow\left(V, v_{0}\right)$ and $p^{\prime}:\left(\tilde{U}, \tilde{u}_{0}\right) \rightarrow\left(U, u_{0}\right)$ be the universal covering maps, let $\bar{V}=\widetilde{V} / H$, and let $\bar{q}:\left(\widetilde{V}, \widetilde{v}_{0}\right) \rightarrow\left(\bar{V}, \bar{q}\left(\widetilde{v}_{0}\right)\right)$ and $\bar{p}:\left(\bar{V}, \bar{q}\left(\widetilde{v}_{0}\right)\right) \rightarrow\left(V, v_{0}\right)$ be the covering maps. As in the proof of Lemma 4.2, we have the commutative diagrams:

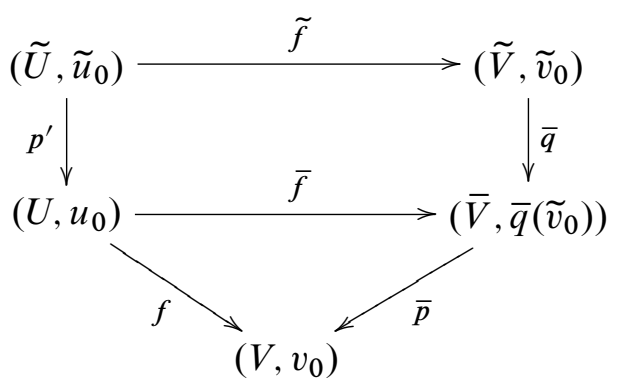

where both $\tilde{f}$ and $\bar{f}$ are embeddings, such that $\tilde{f}(\tilde{U})$ is a simply connected convex submanifold of $\tilde{V}$ covering $\bar{f}(U)$ with covering group $H$. Since $\bar{f}(U)$ is compact, there is a connected compact submanifold $D$ in $\widetilde{V}$ such that $\bar{q}(D)$ contains $\bar{f}(U)$. Since the action of $\Gamma=\pi_{1}\left(V, v_{0}\right)$ on $\tilde{V}$ is properly discontinuous, there are only finitely many elements $\gamma$ of $\Gamma$ with $D \cap \gamma(D) \neq \varnothing$. Let $y_{1}, \ldots, y_{a}$ be all such elements which are not contained in $H$. Suppose that $G$ is a finite index subgroup of $\Gamma$ such that $G$ contains $H$ but does not contain any of $y_{1}, \ldots, y_{a}$. Let $\breve{V}=\tilde{V} / G$. Then the covering map $\breve{q}:\left(\bar{V}, \bar{q}\left(\widetilde{v}_{0}\right)\right) \rightarrow\left(\breve{V}, \breve{q}\left(\bar{q}\left(\widetilde{v}_{0}\right)\right)\right)$ embeds $\bar{f}(U)$ into $\breve{V}$. Let 
$\breve{p}:\left(\breve{V}, \breve{q}\left(\bar{q}\left(\widetilde{v}_{0}\right)\right)\right) \rightarrow\left(V, v_{0}\right)$ be the finite covering map. One can easily check that $f=$ $\breve{p} \circ \breve{q} \circ \bar{f}$ (since $f=\bar{p} \circ \bar{f}$ and $\bar{p}=\breve{p} \circ \breve{q})$. Hence $\breve{f}=\breve{q} \circ \bar{f}:\left(U, u_{0}\right) \rightarrow\left(\breve{V}, \breve{q}\left(\bar{q}\left(\widetilde{v}_{0}\right)\right)\right)$ is a lift of the map $f:\left(U, u_{0}\right) \rightarrow\left(V, v_{0}\right)$ such that $\breve{f}$ is an embedding.

\section{Cusped quasi-Fuchsian surfaces and their convex cores}

Recall that $M$ denotes an arbitrary fixed, connected, orientable, complete, finite-volume, hyperbolic 3-manifold with a single cusp. We consider $M$ as the quotient space of $\mathbb{H}^{3}$ under the action of a fixed, discrete, torsion-free subgroup $\Gamma$ of $P L S_{2}(\mathbb{C})$. A point $a \in S_{\infty}$ is called a parabolic fixed point of a subgroup of $\Gamma$ if $a$ is the fixed point of a parabolic element of the subgroup (note that the trivial element is not considered as a parabolic element). We may assume that the point $\infty$ is a parabolic fixed point of $\Gamma$ (up to replacing $\Gamma$ by a conjugate of $\Gamma$ in $P S L_{2}(\mathbb{C})$, which we may assume has been done). The quotient map $p: \mathbb{H}^{3} \rightarrow M=\mathbb{H}^{3} / \Gamma$ is a fixed universal covering map of $M$. Note that $\Gamma$ acts on $\mathbb{H}^{3}$ isometrically as the covering transformation group, and $p$ is a local isometry. Also $\Gamma$ is isomorphic to the fundamental group of $M$.

Let $C$ be an embedded geometric cusp in $M$, ie $\mathcal{B}=p^{-1}(C)$ is a set of mutually disjoint horoballs in $\mathbb{H}^{3}$ invariant under the action of $\Gamma$. Later, we may need to shrink $C$ if necessary to satisfy some extra conditions. Note that each component of $\mathcal{B}$ is based at a parabolic fixed point of $\Gamma$, and in this fashion the set of parabolic fixed points of $\Gamma$ is in one-to-one correspondence with the set of components of $\mathcal{B}$. Also the set of parabolic fixed points of $\Gamma$ is invariant under the action of $\Gamma$, and the action is transitive (since $M$ has a single cusp). Hence all components of $\mathcal{B}$ are mutually isometric to each other by an element of $\Gamma$.

Let $M^{-}$be the complement of the interior of $C$ in $M$. By Culler-Shalen [9] and Cooper-Long [7] there are two connected, embedded, orientable, cusped, quasiFuchsian surfaces $S_{i}$ in $M$, such that $S_{i}^{-}=S_{i} \cap M^{-}, i=1,2$, have different boundary slopes (we may assume that $S_{i} \cap \partial M^{-}$is a set of embedded simple closed curves each being essential in the torus $\partial M^{-}$). Let $n_{i}$ be the number of cusps in $S_{i}$, ie $n_{i}$ is the number of components of $\partial S_{i}^{-}$. By a well-known duality argument, at least one of the surfaces $S_{i}$ must have even number of boundary components, ie at least one of the integers $n_{i}$ must be even.

Let $\tilde{S}_{i}$ be a fixed component of $p^{-1}\left(S_{i}\right) \subset \mathbb{H}^{3}$ whose closure in $\overline{\mathbb{H}}^{3}$ contains the point $\infty$. Let $\operatorname{Stab}_{\Gamma}\left(\widetilde{S}_{i}\right)$ denote the maximal subgroup of $\Gamma$ which leaves $\widetilde{S}_{i}$ invariant. Then there is a finite-index subgroup $\Gamma_{i}$ of $\operatorname{Stab}_{\Gamma}\left(\widetilde{S}_{i}\right)$ such that $\widetilde{S}_{i} / \Gamma_{i}=S_{i}$ and $\Gamma_{i}$ is isomorphic to the fundamental group of $S_{i}$. As $\Gamma_{i}$ is a quasi-Fuchsian subgroup, the limit set $\Lambda_{i}$ of $\Gamma_{i}$ is a Jordan circle in $S_{\infty}^{2}$, containing the point $\infty$ (by our choice). 
Let $H_{i}$ be the convex hull of $\Lambda_{i}$ in $\mathbb{H}^{3}$. Note that $H_{i}$ is invariant under the action of $\Gamma_{i}$.

Lemma 5.1 [7] The convex hull $H_{i}$ lies between two parallel vertical planes in $\mathbb{H}^{3}$.

The two vertical planes given by Lemma 5.1 are based on two parallel Euclidean lines in $\mathbb{C}$. Among all pairs of planes satisfying Lemma 5.1, let $P_{i, j}, j=1,2$, be the pair which are closest to each other; thus $H_{i}$ lies between $P_{i, 1}$ and $P_{i, 2}$, and $P_{i, j} \cap H_{i}$ is non-empty for each $j=1,2$. Let $W_{i}$ be the closed 3-dimensional region between the two planes $P_{i, 1}$ and $P_{i, 2}$. Let $B_{\infty}$ be the component of $\mathcal{B}$ based at the point $\infty$. So $\partial B_{\infty}$ is a horizontal, Euclidean plane in $\mathbb{H}^{3}$, and $W_{i} \cap \partial B_{\infty}$ is a strip - ie a region bounded by parallel lines in a Euclidean plane. Furthermore, $W_{i} \cap B_{\infty}$ is the product of the strip $W_{i} \cap \partial B_{\infty}$ with $[0, \infty)$; we call this a 3-dimensional strip region, based on $W_{i} \cap \partial B_{\infty}$.

Lemma 5.2 If the cusp $C$ of $M$ is small enough, or equivalently if the horizontal plane $\partial B_{\infty}$ is high enough (ie its Euclidean distance from the complex plane $\mathbb{C}$ is big enough), then $H_{i} \cap B_{\infty}=W_{i} \cap B_{\infty}$.

Proof Since $H_{i}$ is convex, we just need to show that if the horizontal plane $\partial B_{\infty}$ is high enough, then $P_{i, j} \cap B_{\infty}$ is contained in $H_{i}$ for both $j=1,2$. We prove this for $j=1$; the $j=2$ case being entirely similar. Each of $H_{i}, W_{i}, P_{i, 1}$ is invariant under the action of some parabolic element $\beta_{i}$ of $\Gamma_{i}$, which is a horizontal Euclidean translation. Let $x$ be a point in $H_{i} \cap P_{i, 1}$. Then $\beta_{i}(x)$ is also contained in $H_{i} \cap P_{i, 1}$, and so is the hyperbolic geodesic segment $\alpha$ in $P_{i, 1}$ with endpoints $x$ and $\beta_{i}(x)$. Since $\infty$ is a limit point of $H_{i}$, every vertical ray in $\mathbb{H}^{3}$ based at a point in $H_{i}$ is entirely contained in $H_{i}$. So the part of $P_{i, 1}$ lying directly above $\alpha$ is contained in $H_{i} \cap P_{i, 1}$. So all the translations of this set under powers of $\beta_{i}$ are contained in $H_{i} \cap P_{i, 1}$. So it is clear that if $\partial B_{\infty}$ is higher than the highest point of the geodesic segment $\alpha$, then $P_{i, 1} \cap B_{\infty}$ is contained in $H_{i}$.

Note that the center line of the strip $H_{i} \cap \partial B_{\infty}$ has the same slope as that of $\partial S_{i}^{-}$; that is, its image under the covering map $p: \mathbb{H}^{3} \rightarrow M$ is a simple closed curve in $\partial M^{-}$ isotopic to a boundary component of $S_{i}^{-}$.

Now let $B_{a}$ be any fixed component of $\mathcal{B}$ based at a parabolic fixed point $a$ of $\Gamma_{i}$, and let $\gamma \in \Gamma$ be any fixed element which maps $a$ to $\infty$. Then $\gamma\left(B_{a}\right)=B_{\infty}$. Consider the convex set $\gamma\left(H_{i}\right)$. As in Lemma 5.2, one can show that, after shrinking $C$ if necessary, $\gamma\left(H_{i}\right) \cap B_{\infty}$ is a 3-dimensional strip region, based on a strip in $\partial B_{\infty}$. Note that the center line of the strip $\gamma\left(H_{i}\right) \cap \partial B_{\infty}$ is parallel to the center line of the strip $H_{i} \cap \partial B_{\infty}$, since the boundary curves of $S_{i}^{-}$are all isotopic in $\partial M_{i}^{-}$. 
Lemma 5.3 Up to replacing the cusp $C$ by a smaller geometric cusp, $\gamma\left(H_{i}\right) \cap B_{\infty}$ is a 3-dimensional strip region, for every $\gamma \in \Gamma$ which sends a parabolic fixed point of $\Gamma_{i}$ to $\infty$. Moreover the center line of the strip $\gamma\left(H_{i}\right) \cap \partial B_{\infty}$ is parallel to the center line of the strip $H_{i} \cap \partial B_{\infty}$.

Proof The lemma follows from the notes given in the preceding paragraph, together with the facts that the set of parabolic fixed points of $\Gamma_{i}$ is invariant under the action of $\Gamma_{i}$ and that the action has only finitely many orbits (exactly $n_{i}$ orbits in fact).

From now on we assume that the cusp $C$ of $M$ has been chosen small enough so that the conclusion of Lemma 5.3 holds.

Fix a small positive number $\epsilon(\mathrm{eg} \epsilon=1)$ and let $X_{i}$ be the $\epsilon$-collared neighborhood of $H_{i}$ in $\mathbb{H}^{3}$ (cf Lemma 4.3).

Corollary 5.4 $\gamma\left(X_{i}\right) \cap \partial B_{\infty}$ is a strip between two parallel Euclidean lines in $\partial B_{\infty}$ for every $\gamma \in \Gamma$ which sends a parabolic fixed point of $\Gamma_{i}$ to $\infty$. Moreover the center line of the strip $\gamma\left(X_{i}\right) \cap \partial B_{\infty}$ is parallel to the center line of the strip $X_{i} \cap \partial B_{\infty}$.

In fact $\gamma\left(X_{i}\right) \cap \partial B_{\infty}$ is an $\epsilon$-collared neighborhood of $\gamma\left(H_{i}\right) \cap \partial B_{\infty}$ in $\partial B_{\infty}$ for every $\gamma$ given in Corollary 5.4.

Note that $X_{i}$ is a metrically complete and strictly convex 3-submanifold of $\mathbb{H}^{3}$ with $C^{1}$ boundary, invariant under the action of $\Gamma_{i}$. Let

$$
\mathcal{B}_{i}=\left\{X_{i} \cap B ; B \text { a component of } \mathcal{B} \text { based at a parabolic fixed point of } \Gamma_{i}\right\} .
$$

We call $\mathcal{B}_{i}$ the horoball region of $X_{i}$. Let $X_{i}^{-}=X_{i} \backslash \mathcal{B}_{i}$, and call $X_{i}^{-} \cap \partial \mathcal{B}_{i}$ the parabolic boundary of $X_{i}^{-}$, denoted by $\partial_{p} X_{i}^{-}$. Note that $X_{i}^{-}$is locally convex everywhere except on its parabolic boundary.

Each of $X_{i}, \mathcal{B}_{i}, X_{i}^{-}$and $\partial_{p} X_{i}^{-}$is invariant under the action of $\Gamma_{i}$. Let $Y_{i}=X_{i} / \Gamma_{i}$, which is a metrically complete and strictly convex hyperbolic 3-manifold with boundary. Topologically $Y_{i}=S_{i} \times I$, where $I=[-1,1]$. There is a local isometry $f_{i}$ of $Y_{i}$ into $M$, which is induced from the covering map $\mathbb{H}^{3} / \Gamma_{i} \longrightarrow M$ by restriction on $Y_{i}$, since $Y_{i}=X_{i} / \Gamma_{i}$ is a submanifold of $\mathbb{H}^{3} / \Gamma_{i}$. Also $\left.p\right|_{X_{i}}=f_{i} \circ p_{i}$, where $p_{i}$ is the universal covering map $X_{i} \rightarrow Y_{i}=X_{i} / \Gamma_{i}$. Let $Y_{i}^{-}=X_{i}^{-} / \Gamma_{i}$, let $\mathcal{C}_{i}=\mathcal{B}_{i} / \Gamma_{i}$, and let $\partial_{p} Y_{i}^{-}=\partial_{p} X_{i}^{-} / \Gamma_{i}$. We call $\mathcal{C}_{i}$ the cusp part of $Y_{i}$, and call $\partial_{p} Y_{i}^{-}$the parabolic boundary of $Y_{i}^{-}$, which is the frontier of $Y_{i}^{-}$in $Y_{i}$ and is also the frontier of $\mathcal{C}_{i}$ in $Y_{i}$. The manifold $Y_{i}^{-}$is locally convex everywhere except on its parabolic boundary. Topologically $Y_{i}^{-}=S_{i}^{-} \times I$, where each component of $\partial_{p} Y_{i}^{-}$is an annulus. 
From now on we fix an $I$-bundle structure for $Y_{i}=S \times I$ as follows. We first fix an $I$-bundle structure on $Y_{i}^{-}=S_{i}^{-} \times I$ such that $\partial_{p} Y_{i}^{-}=\partial S_{i}^{-} \times I$. We may actually assume that $\partial S_{i}^{-} \times\{0\}$ are the center horo-circles of $\partial_{p} Y_{i}^{-}$and that all the $I$-fibers in $\partial_{p} Y_{i}^{-}$are perpendicular to $\partial S_{i}^{-} \times\{0\}$ with respect to the hyperbolic metric. Next we extend the $I$-bundle structure to the cusp part $\mathcal{C}_{i}$ of $Y_{i}$ in the most natural way, ie if $C_{i, j}$ is a component of $\mathcal{C}_{i}$ and if we write $C_{i, j}$ as $A_{i, j} \times[0, \infty)$, where each $A_{i, j} \times\{*\}$ is a horo-annulus, then we require each $A_{i, j} \times\{*\}$ consists of $I$-fibers, and all the $I$-fibers in $A_{i, j} \times\{*\}$ to be Euclidean geodesics perpendicular to the center horo-circle of $A_{i, j} \times\{*\}$.

We let any (free) cover of $Y_{i}$ have the induced $I$-bundle structure. In particular $X_{i}$ has the induced $I$-bundle structure from that of $Y_{i}$, and this structure is preserved by the action of $\Gamma_{i}$; ie every element of $\Gamma_{i}$ sends an $I$-fiber of $X_{i}$ to an $I$-fiber of $X_{i}$.

Lemma 5.5 For each $i=1,2$, there is a upper bound for the lengths of the $I$-fibers of $Y_{i}$.

Proof Certainly the lengths of the $I$-fibers of $Y_{i}^{-}=S_{i}^{-} \times I$ are bounded, since $S_{i}^{-}$ is compact. So we only need to show that the lengths of the $I$-fibers are bounded in the cusp part $\mathcal{C}_{i}$ of $Y_{i}$. In turn we just need to show that this is true for every component of $\mathcal{C}_{i}$. Let $C_{i, j}$ be a component of $\mathcal{C}_{i}$, and let $\widetilde{C}_{i, j}$ be a component of $p_{i}^{-1}\left(C_{i, j}\right)$. There is an element $\sigma_{i, j}$ of $\Gamma$ such that $\sigma_{i, j}\left(\tilde{C}_{i, j}\right)=\sigma_{i, j}\left(X_{i}\right) \cap B_{\infty}$. So we only need to show that the lengths of the $I$-fibers are bounded in $\sigma_{i, j}\left(X_{i}\right) \cap B_{\infty}$. But $\sigma_{i, j}\left(X_{i}\right) \cap B_{\infty}$ is the $\epsilon$-collared neighborhood of $\sigma_{i, j}\left(H_{i}\right) \cap B_{\infty}$ in $B_{\infty}$ by Lemma 5.3. Also from Lemma 5.3, we see that $\sigma_{i, j}\left(H_{i}\right) \cap B_{\infty}$ has the natural $I$-bundle structure, which is the restriction of the $I$-bundle structure of $\sigma_{i, j}\left(X_{i}\right) \cap B_{\infty}$. Clearly all $I$-fibers of $\sigma_{i, j}\left(H_{i}\right) \cap \partial B_{\infty}$ have the same length and every other $I$-fiber of $\sigma_{i, j}\left(H_{i}\right) \cap B_{\infty}$ has shorter length. Similar conclusions hold for $I$-fibers of $\sigma_{i, j}\left(X_{i}\right) \cap B_{\infty}$.

Corollary 5.6 For each $i=1,2$, there is a upper bound for the lengths of the $I$-fibers of $X_{i}$.

The map $f_{i}: Y_{i}=S_{i} \times I \rightarrow M$ is a local isometry but is not an embedding in general. In particular the center surface $f_{i} \mid: S_{i} \times\{0\} \rightarrow M$ may not be an embedding, but it follows from Corollary 5.4 that the map is an embedding when restricted on each component of $\left(S_{i} \times\{0\}\right) \cap \mathcal{C}_{i}$. Hence we may slightly perturb, if necessary, the cusp part of the $S_{i} \times\{0\}$ in $Y_{i}$, keeping it totally geodesic and transverse to the $I$-fibers, so that the resulting surface, when restricted to its cusp part, will be an embedding under the map $f_{i}$. We still use $S_{i}$ to denote this surface, and we still denote $Y_{i}$ as $S_{i} \times I$ and $Y_{i}^{-}$as $S_{i}^{-} \times I$. We call $S_{i}$ the (topological) center surface of $Y_{i}$. Note 
that $f_{i}: S_{i} \rightarrow M$ is quasi-Fuchsian and each component of $p^{-1}\left(f_{i}\left(S_{i}\right)\right)$ is contained in $\gamma\left(X_{i}\right)$ as a topological center surface for some $\gamma \in \Gamma$.

The restriction map $f_{i}:\left(Y_{i}^{-}, \partial_{p} Y_{i}^{-}\right) \rightarrow\left(M^{-}, \partial M^{-}\right)$is a proper map of pairs and $f_{i} \mid:\left(S_{i}^{-}, \partial S_{i}^{-}\right) \rightarrow\left(M^{-}, \partial M^{-}\right)$is a proper map which is an embedding on $\partial S_{i}^{-}$(This property will remain valid if we shrink the cusp $C$ of $M$ geometrically). We fix an orientation for $S_{i}$, and let $S_{i}^{-}$and $\partial S_{i}^{-}$have the induced orientation. Let $\beta_{i, j}$, $j=1, \ldots, n_{i}$, denote the components of $\partial S_{i}$ indexed so that their images $f_{i}\left(\beta_{i, j}\right)$, $j=1, \ldots, n_{i}$, appear consecutively on $\partial M^{-}$. Let $\Delta$ be the geometric intersection number between $f_{1}\left(\beta_{1,1}\right)$ and $f_{2}\left(\beta_{2,1}\right)$. Since each $f_{i}\left(\beta_{i, j}\right)$ is a Euclidean circle in the Euclidean torus $\partial M^{-}$, each pair of circles $f_{1}\left(\beta_{1, j}\right)$ and $f_{2}\left(\beta_{2, k}\right)$ have exactly $\Delta$ intersect points. Hence there are a total of $d=n_{1} n_{2} \Delta$ intersection points between $f_{1}\left(\partial S_{1}^{-}\right)$and $f_{2}\left(\partial S_{2}^{-}\right)$in the torus $\partial M^{-}$(all distinct in $\left.\partial M^{-}\right)$. Let $t_{1}, \ldots, t_{d}$ denote these intersection points. The points $f_{i}^{-1}\left\{t_{1}, \ldots, t_{d}\right\}$ can be indexed as $\left\{t_{i, j, k}, j=\right.$ $\left.1, \ldots, n_{i}, k=1, \ldots, d_{i}\right\}$, where $d_{i}=\Delta n_{i_{*}}$ and $i_{*}$ is the number such that $\left\{i, i_{*}\right\}=$ $\{1,2\}$. We may further assume that $\left\{t_{i, j, k}, k=1, \ldots, d_{i}\right\}$ are contained successively in the component $\beta_{i, j}$, following the orientation of $\beta_{i, j}$, for each $j=1, \ldots, n_{i}$.

We remark that all the results and notations in this section will still be valid and consistent if we replace the cusp $C$ by a smaller one.

\section{The manifold $K_{i}$}

We continue to use the notations established in Section 5. The purpose of this section is to construct, for each of $i=1,2$, a manifold $K_{i}$, which, on an intuitive level, corresponds to the intersection of $Y_{1}$ and $Y_{2}$ in $M$, and which will be used to cut and paste two immersions.

For each of the points $t_{j}, j=1, \ldots, d$, which was defined at the end of Section 5, there is a unique embedded geodesic ray $R_{j}$ in $C$, based at $t_{j}$, perpendicular to $\partial C$. We shall associate to each $R_{j}$ (thus to $t_{j}$ ), a metrically complete and convex hyperbolic manifold $K_{i, k}$ with a local isometry, $g_{i, k}$, into $Y_{i}$ (for each of $i=1,2$ ) such that:

(1) the truncated version of $K_{i, k}$, denoted $K_{i, k}^{-}$(whose definition will be given below), is a compact 3 -manifold;

(2) there is an isometry $h_{k}: K_{1, k} \rightarrow K_{2, k}$ such that

$$
h_{k} \mid:\left(K_{1, k}^{-}, \partial_{p} K_{1, k}^{-}\right) \rightarrow\left(K_{2, k}^{-}, \partial_{p} K_{2, k}^{-}\right)
$$

is a proper isometry. (The definition of $\partial_{p} K_{i, k}^{-}$will be given below.) 
To do this, we first choose points $b_{j}, j=1, \ldots, d$, in $\partial B_{\infty}$ such that $p\left(b_{j}\right)=t_{j}$. Recall that $p: \mathbb{H}^{3} \rightarrow M$ and $p_{i}: X_{i} \rightarrow Y_{i}$ are fixed universal covering maps. Let $\widetilde{S}_{i}=p_{i}^{-1}\left(S_{i}\right)$. Then $\tilde{S}_{i}$ is the (topological) center surface of $X_{i}$. Since $\Gamma$ acts transitively on the set $p^{-1}\left(t_{j}\right)$ for each fixed $j$, there is an element $\gamma_{i, j}$ of $\Gamma$ such that $\gamma_{i, j}\left(\tilde{S}_{i}\right)$ contains the point $b_{j}$. Let $X_{i, j}=\gamma_{i, j}\left(X_{i}\right)$. Then $\gamma_{i, j}\left(\tilde{S}_{i}\right)$ is the center surface of $X_{i, j}=\gamma_{i, j}\left(X_{i}\right)$, and $X_{i, j}$ is invariant under the action of the subgroup $\gamma_{i, j} \Gamma_{i} \gamma_{i, j}^{-1}$. Let $W_{j}=X_{1, j} \cap X_{2, j}$. Then $W_{j}$ is a metrically complete and strictly convex (thus simply connected) 3-dimensional submanifold of $\mathbb{H}^{3}$ which is invariant under the action of the subgroup $\left(\gamma_{1, j} \Gamma_{1} \gamma_{1, j}^{-1}\right) \cap\left(\gamma_{2, j} \Gamma_{2} \gamma_{2, j}^{-1}\right)$. Let $Z_{i, j}=\gamma_{i, j}^{-1}\left(W_{j}\right)$. Then $Z_{1, j}=X_{1} \cap \gamma_{1, j}^{-1} \gamma_{2, j}\left(X_{2}\right)$ is contained in $X_{1}$ and is invariant under the action of the subgroup $\Gamma_{i, j}=\Gamma_{1} \cap\left(\gamma_{1, j}^{-1} \gamma_{2, j} \Gamma_{2} \gamma_{2, j}^{-1} \gamma_{1, j}\right)$, and similarly $Z_{2, j}=X_{2} \cap \gamma_{2, j}^{-1} \gamma_{1, j}\left(X_{1}\right)$ is contained in $X_{2}$ and is invariant under the action of the subgroup $\Gamma_{2, j}=\Gamma_{2} \cap$ $\left(\gamma_{2, j}^{-1} \gamma_{1, j} \Gamma_{1} \gamma_{1, j}^{-1} \gamma_{2, j}\right)$.

Lemma 6.1 The subgroup $\Gamma_{i, j}$ contains no parabolic elements, for any $i=1,2, j=$ $1, \ldots, d$.

Proof Recall that $i$ and $i_{*}$ denote the number 1 or 2 such that $\left\{i, i_{*}\right\}=\{1,2\}$, and that $\Gamma_{i, j}=\Gamma_{i} \cap\left(\gamma_{i, j}^{-1} \gamma_{i_{*}, j} \Gamma_{i_{*}} \gamma_{i_{*}, j}^{-1} \gamma_{i, j}\right)$. Also recall $\Gamma_{i}, i=1,2$, are the fundamental groups of two embedded, cusped, quasi-Fuchsian surfaces, with different boundary slopes. Thus no parabolic element in $\Gamma_{i}$ is conjugate in $\Gamma$ to any element in $\Gamma_{i_{*}}$ (cf the proof of Cooper-Long [7, Lemma 2.1]). Hence the conclusion of the lemma follows.

Recall that $\mathcal{B}_{i}$ is the horoball region of $X_{i}$, which is the intersection of $X_{i}$ with the collection of horoballs in $\mathcal{B}$ based at parabolic fixed points of $\Gamma_{i}$. Note that $\Lambda\left(X_{i}\right)=\Lambda\left(\Gamma_{i}\right)$. We claim that the limit set $\Lambda\left(Z_{i, j}\right)$ of $Z_{i, j}$ is equal to the intersection $\Lambda\left(\Gamma_{i}\right) \cap \Lambda\left(\gamma_{i, j}^{-1} \gamma_{i_{*}, j} \Gamma_{i_{*}} \gamma_{i_{*}, j}^{-1} \gamma_{i, j}\right)$. Indeed, the containment $\Lambda\left(Z_{i, j}\right) \subset$ $\Lambda\left(\Gamma_{i}\right) \cap \Lambda\left(\gamma_{i, j}^{-1} \gamma_{i_{*}, j} \Gamma_{i_{*}} \gamma_{i_{*}, j}^{-1} \gamma_{i, j}\right)$ is obvious. For the other containment, suppose that $x$ is in $\Lambda\left(\Gamma_{i}\right) \cap \Lambda\left(\gamma_{i, j}^{-1} \gamma_{i_{*}, j} \Gamma_{i_{*}} \gamma_{i_{*}, j}^{-1} \gamma_{i, j}\right)$. Then there are geodesic rays $\alpha$ and $\alpha^{\prime}$, contained in $H_{i}$ and $\gamma_{i, j}^{-1} \gamma_{i_{*}, j} H_{i_{*}}$ respectively, with $x$ as a limit endpoint. Then far enough along these geodesics, each point in $\alpha$ is within an epsilon-neighborhood of $\alpha^{\prime}$, and vice versa. Therefore, far enough along these geodesics, $\alpha$ and $\alpha^{\prime}$ are both contained in $X_{i} \cap \gamma_{i, j}^{-1} \gamma_{i_{*}, j} X_{i_{*}}$, and therefore $x$ is a limit point of $Z_{i, j}$.

Since quasi-Fuchsian groups are geometrically finite, we are able to apply MatsuzakiTaniguchi [14, Theorem 3.14] (which is originally due to Susskind [18]) and to conclude that $\Lambda\left(\Gamma_{i}\right) \cap \Lambda\left(\gamma_{i, j}^{-1} \gamma_{i_{*}, j} \Gamma_{i_{*}} \gamma_{i_{*}, j}^{-1} \gamma_{i, j}\right)=\Lambda\left(\Gamma_{i, j}\right) \cup P_{i, j}$, where $P_{i, j}$ is the set of points $\zeta \in \Omega\left(\Gamma_{i, j}\right)=S_{\infty}^{2}-\Lambda\left(\Gamma_{i, j}\right)$ such that: 
(1) $\operatorname{Stab}_{\Gamma_{i}}(\zeta)$ and $\operatorname{Stab}_{\gamma_{i, j}^{-1} \gamma_{i_{*}, j} \Gamma_{i *} \gamma_{i_{*}, j}^{-1} \gamma_{i, j}}(\zeta)$ generate a rank two Abelian group;

(2) $\operatorname{Stab}_{\Gamma_{i}}(\zeta) \cap \operatorname{Stab}_{\gamma_{i, j}^{-1} \gamma_{i_{*}, j} \Gamma_{i *} \gamma_{i_{*}, j}^{-1} \gamma_{i, j}}(\zeta)=\{\mathrm{id}\}$

Also, $\Lambda\left(\Gamma_{i, j}\right)=\Lambda_{p}\left(\Gamma_{i, j}\right) \cup \Lambda_{c}\left(\Gamma_{i, j}\right)$, where $\Lambda_{c}$ denotes the set of conical limit points and $\Lambda_{p}$ the set of parabolic limit points (see Matsuzaki-Taniguchi [14] or Ratcliffe [17] for their definitions). By Lemma 6.1, $\Gamma_{i, j}$ contains no parabolic elements, and thus $\Lambda_{p}\left(\Gamma_{i, j}\right)=\varnothing$. Thus $\Lambda\left(\Gamma_{i}\right) \cap \Lambda\left(\gamma_{i, j}^{-1} \gamma_{i_{*}, j} \Gamma_{i_{*}} \gamma_{i_{*}, j}^{-1} \gamma_{i, j}\right)=\Lambda_{c}\left(\Gamma_{i, j}\right) \cup P_{i, j}$.

Let $\mathcal{B}_{i, j}$ be the intersection of $Z_{i, j}$ with the collection of horoballs in $\mathcal{B}$ based at points of $P_{i, j}$. We call $\mathcal{B}_{i, j}$ the horoball region of $Z_{i, j}$. Let $Z_{i, j}^{-}=Z_{i, j} \backslash \mathcal{B}_{i, j}$, which is the truncated version of $Z_{i, j}$. We call $Z_{i, j}^{-} \cap \partial \mathcal{B}_{i, j}$ the parabolic boundary of $Z_{i, j}^{-}$ and denote it by $\partial_{p} Z_{i, j}^{-}$. Note that $Z_{i, j}^{-}$is locally convex everywhere except on its parabolic boundary. Each of $Z_{i, j}, \mathcal{B}_{i, j}, Z_{i, j}^{-}$and $\partial_{p} Z_{i, j}^{-}$is invariant under the action of $\Gamma_{i, j}$.

Some members of $\left\{Z_{i, 1}, \ldots, Z_{i, d}\right\}$ maybe the same submanifold of $X_{i}$ modulo the action of $\Gamma_{i}$ on $X_{i}$, ie some one maybe a translation of another by an element of $\Gamma_{i}$.

Lemma 6.2 The equality $Z_{1, j}=\gamma_{1}\left(Z_{1, k}\right)$ holds for some $\gamma_{1} \in \Gamma_{1}$ if and only if $Z_{2, j}=\gamma_{2}\left(Z_{2, k}\right)$ for some $\gamma_{2} \in \Gamma_{2}$.

Proof Let $Z_{1, j}=\gamma_{1}\left(Z_{1, k}\right)$ for some element $\gamma_{1} \in \Gamma_{1}$ and suppose that $\gamma_{2}=$ $\left(\gamma_{2, j}^{-1} \gamma_{1, j}\right) \gamma_{1}\left(\gamma_{1, k}^{-1} \gamma_{2, k}\right)$. Then by our construction, $\gamma_{2}$ maps $Z_{2, k}$ to $Z_{2, j}$. Also, $Z_{2, k}$ contains a point in $p^{-1}\left(t_{k}\right) \cap \widetilde{S}_{2}$, and $\gamma_{2}$ maps this to another point in $p^{-1}\left(t_{k}\right) \cap \widetilde{S}_{2}$. Since the $\Gamma$-stabilizer of any point in $p^{-1}\left(t_{k}\right)$ is trivial and since $\Gamma_{2}$ acts transitively on the set $p^{-1}\left(t_{k}\right) \cap \widetilde{S}_{2}$, the element $\gamma_{2}$ must belong to $\Gamma_{2}$.

Let $j_{1}, \ldots, j_{q}$ be such that $\left\{Z_{i, j_{1}}, \ldots, Z_{i, j_{q}}\right\}$ is a maximal set of representatives of $\left\{Z_{i, 1}, \ldots, Z_{i, d}\right\}$ which are mutually inequivalent under the action of $\Gamma_{i}$ on $X_{i}$ for each $i=1,2$. Note that the set $\left\{Z_{i, j_{1}}, \ldots, Z_{i, j_{q}}\right\}$ is well defined (independent of the choices for the points $\left.b_{j} \in p^{-1}\left(t_{j}\right) \cap \partial B_{\infty}\right)$, up to translations by elements in $\Gamma_{i}$.

Lemma 6.3 The subgroup $\Gamma_{i, j_{k}}$ acts transitively on $p^{-1}\left(t_{j}\right) \cap Z_{i, j_{k}} \cap \widetilde{S}_{i}$, for each fixed $j, k, i$.

Proof We know that $\Gamma_{i}$ acts transitively on $p^{-1}\left(t_{j}\right) \cap \widetilde{S}_{i}$ and $\gamma_{i, j_{k}}^{-1} \gamma_{i_{*}, j_{k}} \Gamma_{i_{*}} \gamma_{i_{*}, j_{k}}^{-1} \gamma_{i, j_{k}}$ acts transitively on $p^{-1}\left(t_{j}\right) \cap \gamma_{i, j_{k}}^{-1} \gamma_{i_{*}, j_{k}}\left(\tilde{S}_{i_{*}}\right)$, so given two distinct points $\tilde{t}$ and $\tilde{t}^{\prime}$ in $p^{-1}\left(t_{j}\right) \cap Z_{i, j_{k}} \cap \widetilde{S}_{i}$, there exists $\gamma \in \Gamma_{i}$, and $\gamma^{\prime} \in \gamma_{i, j_{k}}^{-1} \gamma_{i_{*}, j_{k}} \Gamma_{i_{*}} \gamma_{i_{*}, j_{k}}^{-1} \gamma_{i, j_{k}}$ such that each of them maps $\tilde{t}$ to $\widetilde{t}^{\prime}$. But there is a unique element of $\Gamma$ which maps $\tilde{t}$ to $\widetilde{t}^{\prime}$. Thus $\gamma=\gamma^{\prime}$ and so $\gamma \in \Gamma_{i} \cap\left(\gamma_{i, j_{k}}^{-1} \gamma_{i_{*}, j_{k}} \Gamma_{i_{*}} \gamma_{i_{*}, j_{k}}^{-1} \gamma_{i, j_{k}}\right)=\Gamma_{i, j_{k}}$. 
Each of the manifolds $Z_{i, j_{k}}, Z_{i, j_{k}}^{-}, \partial_{p} Z_{i, j_{k}}^{-}$and $\mathcal{B}_{i, j_{k}}$ is invariant under the action of the subgroup:

$$
\Gamma_{i, j_{k}}=\Gamma_{i} \cap\left(\gamma_{i, j_{k}}^{-1} \gamma_{i_{*}, j_{k}} \Gamma_{i_{*}} \gamma_{i_{*}, j_{k}}^{-1} \gamma_{i, j_{k}}\right)
$$

Let $K_{i, k}=Z_{i, j_{k}} / \Gamma_{i, j_{k}}, K_{i, k}^{-}=Z_{i, j_{k}}^{-} / \Gamma_{i, j_{k}}, \partial_{p} K_{i, k}=\partial_{p} Z_{i, j_{k}}^{-} / \Gamma_{i, j_{k}}$, and $\mathcal{C}_{i, k}=$ $\mathcal{B}_{i, j_{k}} / \Gamma_{i, j_{k}}$. For each $k=1, \ldots, q, K_{1, k}$ and $K_{2, k}$ are isometric, metrically complete, convex, hyperbolic manifolds. The isometry from $K_{1, k}$ to $K_{2, k}$ is the map $h_{k}$ which makes the following diagram commute:

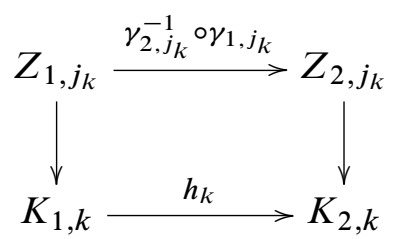

where the vertical maps are the covering maps. Also for each $i$ and $k$, there is a local isometry $g_{i, k}$ from $K_{i, k}$ into $Y_{i}$ which is the restriction of the covering map $X_{i} / \Gamma_{i, j_{k}} \rightarrow Y_{i}$. Let $K_{i}$ be the disjoint union of $\left\{K_{i, k}, k=1, \ldots, q\right\}$. We have the isometry $h: K_{1} \rightarrow K_{2}$ with $\left.h\right|_{K_{1, k}}=h_{k}$. We also have the local isometry $g_{i}: K_{i} \rightarrow Y_{i}$ with $\left.g_{i}\right|_{K_{i, k}}=g_{i, k}$.

Lemma 6.4 The restriction of the covering map $Z_{i, j_{k}}^{-} \rightarrow K_{i, k}^{-}$to every component of $\partial_{p} Z_{i, j_{k}}^{-}$is an isometric embedding, for each of $i=1,2$ and each of $k=1, \ldots, q$. In fact the restriction of the covering map $Z_{i, j_{k}} \rightarrow K_{i, k}$ to every component of $\mathcal{B}_{i, j_{k}}$ is an isometric embedding, for each of $i=1,2$ and each of $k=1, \ldots, q$.

Proof It follows from Corollary 5.4 and the transitivity of the action of $\Gamma$ on the set $p^{-1}\left(t_{j}\right)$ (for any fixed $j$ ) that every component of $\partial_{p} Z_{i, j_{k}}^{-}$is a Euclidean parallelogram in some horosphere. Now the first statement of the lemma follows from the fact that $\Gamma_{i, j_{k}}$ has no parabolic elements (Lemma 6.1). The second assertion can be proved similarly.

We have just shown that each component $\widetilde{D}$ of $\partial_{p} Z_{i, j_{k}}^{-}$(for any $i, j_{k}$ ) is a Euclidean parallelogram in a horosphere. We define the (topological) center point of $\widetilde{D}$ to be the point $\widetilde{D} \cap \widetilde{S}_{i} \cap \gamma_{i, j_{k}}^{-1} \gamma_{i_{*}, j_{k}}\left(\widetilde{S}_{i_{*}}\right)$. The union of all the center points in $\partial_{p} Z_{i, j_{k}}^{-}$is invariant under the action of the subgroup $\Gamma_{i, j_{k}}$. By Lemma 6.4, each component $D$ of $\partial_{p} K_{i, k}$ is the isometric image of a component $\widetilde{D}$ of $\partial_{p} Z_{i, j_{k}}^{-}$under the covering map $Z_{i, j_{k}} \rightarrow K_{i, k}$. We define the (topological) center point of $D$ to be the image of the center point of $\widetilde{D}$. Thus by our construction, for each $t_{j} \in f_{1}\left(\partial S_{1}^{-}\right) \cap f_{2}\left(\partial S_{2}^{-}\right)$, there is a component $D$ of $\partial_{p} K_{i, k}^{-}$(for some $k$ ) whose center point is mapped to the point $t_{j}$ under the map $K_{i, k} \rightarrow Y_{i} \rightarrow M$. In fact there is a geodesic ray, based at the 
center point, in the cusp part of $K_{i, k}$ which maps isometrically to the ray $R_{j} \subset C$, under the map $K_{i, k} \rightarrow Y_{i} \rightarrow M$. This component of $\partial_{p} K_{i, k}^{-}$is said to be associated to the ray $R_{j}$ (thus to the point $t_{j}$ ), and so is the component $K_{i, k}$ of $K_{i}$.

Lemma 6.5 For each of $i=1,2$, the parabolic boundary of $K_{i}^{-}$has exactly $d$ components (each being a Euclidean parallelogram), associated to the points $t_{j}, j=$ $1, \ldots, d$, respectively.

Proof We prove this for $i=1$; the case for $i=2$ can be proved similarly. By the construction, we see that the parabolic boundary of $K_{1}^{-}$has at least $d$ components, associated to the points $t_{1}, \ldots, t_{d}$ respectively. Suppose that there are distinct components $P_{1}$ and $P_{2}$ of the parabolic boundary of $K_{1}^{-}$associated to the same point, say $t_{1}$. Also we may assume that $K_{1,1}^{-}$and $K_{1, k}^{-}$are the components of $K_{1}^{-}$containing $P_{1}$ and $P_{2}$ respectively. We first show that $k=1$ is impossible. So suppose that both $P_{1}$ and $P_{2}$ are components of $\partial_{p} K_{1,1}^{-}$. Recall that $K_{1,1}^{-}=Z_{1, j_{1}}^{-} / \Gamma_{1, j_{1}}$ and $\Gamma_{1, j_{1}}=\Gamma_{1} \cap \gamma_{1, j_{1}}^{-1} \gamma_{2, j_{1}} \Gamma_{2} \gamma_{2, j_{1}}^{-1} \gamma_{1, j_{1}}$. So the parabolic boundary of $Z_{1, j_{1}}^{-}$contains two components $\widetilde{P}_{1}$ and $\widetilde{P}_{2}$ which are mapped to $P_{1}$ and $P_{2}$, respectively, under the covering map $Z_{1, j_{1}}^{-} \rightarrow K_{1,1}^{-}$. Because the center points of $\widetilde{P}_{1}$ and $\widetilde{P}_{2}$ are contained in $p^{-1}\left(t_{1}\right)$ and because $\Gamma_{1, j_{1}}$ acts on $p^{-1}\left(t_{1}\right) \cap Z_{1, j_{1}}^{-} \cap \widetilde{S}_{1}$ transitively (Lemma 6.3), there is an element $\gamma \in \Gamma_{1, j_{1}}$ such that $\gamma\left(\widetilde{P}_{1}\right)=\widetilde{P}_{2}$. Hence both $\widetilde{P}_{1}$ and $\widetilde{P}_{2}$ are mapped to $P_{1}$ under the covering map $Z_{1, j_{1}}^{-} \rightarrow K_{1,1}^{-}$, which gives a contradiction. Now suppose that $k \neq 1$. Then $Z_{1, j_{1}}^{-}=X_{1}^{-} \cap \gamma_{1, j_{1}}^{-1} \gamma_{2, j_{1}}\left(X_{2}^{-}\right)$and $Z_{1, j_{k}}^{-}=X_{1}^{-} \cap \gamma_{1, j_{k}}^{-1} \gamma_{2, j_{k}}\left(X_{2}^{-}\right)$ are two different submanifolds of $X_{1}^{-}$, and there are two components $\widetilde{P}_{1}$ and $\widetilde{P}_{2}$, belonging to $\partial_{p} Z_{1, j_{1}}^{-}$and $\partial_{p} Z_{1, j_{k}}^{-}$respectively, which are mapped to $P_{1}$ and $P_{2}$ under the covering maps $Z_{1, j_{1}}^{-} \rightarrow K_{1,1}^{-}$and $Z_{1, j_{k}}^{-} \rightarrow K_{1, k}^{-}$respectively. Since the center points of $\widetilde{P}_{1}$ and $\widetilde{P}_{2}$ are contained in $p^{-1}\left(t_{1}\right)$, and $\Gamma_{1}$ acts on $p^{-1}\left(t_{1}\right) \cap \widetilde{S}_{1}$ transitively, there is an element $\gamma \in \Gamma_{1}$ which maps $\widetilde{P}_{1}$ to $\widetilde{P}_{2}$. So $\gamma \gamma_{1, j_{1}}^{-1} \gamma_{2, j_{1}}\left(X_{2}\right)$ intersects $X_{1}$ at $\widetilde{P}_{2}$. So $\left(\gamma \gamma_{1, j_{1}}^{-1} \gamma_{2, j_{1}}\right)^{-1}\left(\widetilde{P}_{2}\right)$ and $\left(\gamma_{1, j_{k}}^{-1} \gamma_{2, j_{k}}\right)^{-1}\left(\widetilde{P}_{2}\right)$ are both contained in $X_{2}$. It follows that $\left(\gamma \gamma_{1, j_{1}}^{-1} \gamma_{2, j_{1}}\right)\left(\gamma_{1, j_{k}}^{-1} \gamma_{2, j_{K}}\right)^{-1}$ is contained in $\Gamma_{2}$. Therefore $\left(\gamma \gamma_{1, j_{1}}^{-1} \gamma_{2, j_{1}}\right)\left(\gamma_{1, j_{k}}^{-1} \gamma_{2, j_{k}}\right)^{-1}\left(X_{2}\right)=X_{2}$, ie $\gamma \gamma_{1, j_{1}}^{-1} \gamma_{2, j_{1}}\left(X_{2}\right)=\gamma_{1, j_{k}}^{-1} \gamma_{2, j_{k}}\left(X_{2}\right)$. Hence $\gamma\left(Z_{1, j_{1}}\right)=X_{1} \cap \gamma \gamma_{1, j_{1}}^{-1} \gamma_{2, j_{1}}\left(X_{2}\right)=X_{1} \cap \gamma_{1, j_{k}}^{-1} \gamma_{2, j_{k}}\left(X_{2}\right)=Z_{1, j_{k}}$. Hence $Z_{1, j_{1}}$ and $Z_{1, j_{k}}$ are equivalent under the translations of $\Gamma_{1}$. This gives a contradiction to our assumption that these $Z_{1, j_{k}}, k=1, \ldots, q$, are mutually inequivalent under translations of elements of $\Gamma_{1}$.

Lemma 6.6 $K_{i, k}^{-}$is compact for each $i=1,2, k=1, \ldots, q$. 
Proof Recall that $K_{i, k}=Z_{i, j_{k}} /\left(\Gamma_{i, j_{k}}\right)$ and $\Gamma_{i, j_{k}}=\Gamma_{i} \cap\left(\gamma_{i, j_{k}}^{-1} \gamma_{i_{*}, j_{k}} \Gamma_{i_{*}} \gamma_{i_{*}, j_{k}}^{-1} \gamma_{i, j_{k}}\right)$. The limit set of $Z_{i, j_{k}}$ is equal to $\Lambda_{c}\left(\Gamma_{i, j_{k}}\right) \cup P_{i, j_{k}}$ (see the two paragraphs following the proof of Lemma 6.1).

Since $K_{i, k}$ is convex and metrically complete, between any two points in $K_{i, k}$ there is a distance minimizing geodesic connecting them. Fix a point $k_{0}$ in $K_{i, k}^{-}$. Consider $N_{\left(r, K_{i, k}\right)}\left(k_{0}\right)$, the closed $r$-neighborhood of the point $k_{0}$ in $K_{i, j}$. Then by the HopfRinow Theorem (Canary-Epstein-Green [5, Theorem 1.3.]) $N_{\left(r, K_{i, k}\right)}\left(k_{0}\right)$ is a compact subset of $K_{i, k}$ for any $r>0$.

As a subset of $K_{i, k}, K_{i, k}^{-}$is closed. Hence $K_{i, k}^{-} \cap N_{\left(r, K_{i, k}\right)}\left(k_{0}\right)$ is a closed subset of the compact set $N_{\left(r, K_{i, k}\right)}\left(k_{0}\right)$ and thus is compact. Therefore if $K_{i, k}^{-}$is not compact, then it is not contained in $N_{\left(r, K_{i, k}\right)}\left(k_{0}\right)$ for any fixed $r>0$. So we can find a point $k_{r}$ in $K_{i, k}^{-}$with $d\left(k_{0}, k_{r}\right)>r$ for any $r>0$. By Lemma 6.5, the parabolic boundary of $K_{i, k}^{-}$has finitely many components. Also each component of the parabolic boundary of $K_{i, k}^{-}$is a compact Euclidean parallelogram. Hence for all sufficiently large $r>0$, the parabolic boundary of $K_{i, k}^{-}$is contained in $N_{\left(r, K_{i, k}\right)}\left(k_{0}\right)$. Hence the points $k_{r}$ are not in $\partial_{p}\left(K_{i, k}^{-}\right)$for all sufficiently large $r>0$. Let $\alpha_{r}$ be the distance minimizing geodesic segment in $K_{i, k}$ with endpoints $k_{0}$ and $k_{r}$.

Let $p_{i, k}: Z_{i, j_{k}} \rightarrow K_{i, k}$ be the covering map. Pick a point $z_{0}$ in $Z_{i, j_{k}}$ such that $p_{i, k}\left(z_{0}\right)=k_{0}$. Let $\tilde{\alpha}_{r} \subset Z_{i, j_{k}}$ be the lift of $\alpha_{r}$ starting at $z_{0}$ (note that the lift is unique). Let $z_{r}$ be the other endpoint of $\widetilde{\alpha}_{r}$. Note that $\widetilde{\alpha}_{r}$ is a distance minimizing geodesic segment in $Z_{i, j_{k}}$ with $d\left(z_{0}, z\right)=d\left(p_{i, k}\left(z_{0}\right), p_{i, k}(z)\right)$ for any $z \in \tilde{\alpha}_{r}$. Now consider the sequence of the geodesic segments $\left\{\widetilde{\alpha}_{n}\right\}_{n=1}^{\infty}$. As $d\left(z_{0}, z_{n}\right)=d\left(k_{0}, k_{n}\right) \rightarrow+\infty$ as $n \rightarrow+\infty$, there is a subsequence of $\left\{z_{n}\right\}$ which converges to a point $a$ in $S_{\infty}^{2}$. We may assume, for simplicity in notation, that $\left\{z_{n}\right\}$ itself converges to $a$. Now $a$ is in the limit set of $Z_{i, j_{k}}$ and thus $a \in \Lambda_{c}\left(\Gamma_{i, j_{k}}\right) \cup P_{i, j_{k}}$.

Let $\tilde{\alpha}$ be the geodesic ray in $\mathbb{H}^{3}$ starting at $z_{0}$ and approaching $a$ (such a ray exists and is unique). Since $Z_{i, j_{k}}$ is metrically complete and convex, $\tilde{\alpha}$ is contained in $Z_{i, j_{k}}$. In fact the sequence $\left\{\tilde{\alpha}_{n}\right\}$ is approaching $\tilde{\alpha}$ in the sense that every point $x$ in $\tilde{\alpha}$ is the limit of a sequence of points $\left\{x_{n}\right\}$ with $x_{n} \in \widetilde{\alpha}_{n}$. It follows that each finite sub-segment of the projection $p_{i, k}(\widetilde{\alpha})$ is a distance-minimizing segment in $K_{i, k}$.

We first show that the point $a$ is not in $P_{i, j_{k}}$. For otherwise if $B_{a}$ is the horoball component in $\mathcal{B}$ based at $a, \widetilde{\alpha}$ intersects perpendicularly every horosphere inside $B_{a}$ based at $a$. Hence $\widetilde{\alpha} \cap B_{a}$ is a geodesic ray contained in $B_{a} \cap Z_{i, j_{k}}$. But $\widetilde{\alpha}_{n}$ is approaching $\tilde{\alpha}$, so for sufficiently large $n, z_{n}$ will enter into $B_{a}$. So $z_{n}$ is not in $Z_{i, j_{k}}^{-}$ and thus $p_{i, k}\left(z_{n}\right)=k_{n}$ is not contained in $K_{i, k}^{-}$, which contradicts our construction of $k_{n}$. 
So $a$ is a conical limit point of $\Gamma_{i, j_{k}}$. By definition there is a geodesic ray $\tilde{l}$ ending at $a$ and there is a sequence of elements $\sigma_{m}$ in $\Gamma_{i, j_{k}}$ such that $\sigma_{m}\left(z_{0}\right)$ is contained in $N_{\epsilon}(\widetilde{l})$ (which is a fixed $\epsilon$-collared neighborhood of $\tilde{l}$ in $\mathbb{H}^{3}$ ) for all sufficiently large $m$, and converges to the point $a$ as $m \rightarrow \infty$. Now the ray $\tilde{\alpha}$ is contained in $N_{\epsilon}(\widetilde{l})$ except possibly for a finite initial segment. Let $\widetilde{\alpha}_{w}$ be a sub-ray of $\widetilde{\alpha}$ starting at a point $w \in \tilde{\alpha}$ such that $\tilde{\alpha}_{w}$ is entirely contained in $N_{\epsilon}(\tilde{l})$ and $d\left(z_{0}, w\right)>2 \epsilon$. For any point $x$ on $\tilde{l}$ let $P_{x}$ be the hyperbolic plane intersecting $\tilde{l}$ perpendicularly at the point $x$, and let $D_{x}=P_{x} \cap N_{\epsilon}(\widetilde{l})$. Then $D_{x}$ is topologically a disk separating $N_{\epsilon}(\widetilde{l})$ into two pieces one of which contains the sub-ray of $\tilde{l}$ starting at $x$. Now every point in the ray $\widetilde{\alpha}_{w}$ is contained in $D_{x}$ for some $x \in \tilde{l}$. In particular the endpoint $w$ of $\tilde{\alpha}_{w}$ is contained in some $D_{x}$. Let $V$ be the component of $N_{\epsilon}(\tilde{l})-D_{x}$ which contains a sub-ray of $\tilde{l}$. Since the sequence $\left(\sigma_{m}\left(z_{0}\right)\right)$ approaches $a$, there is some $\sigma_{m}\left(z_{0}\right)$ which is contained in $V$. Let $D_{x^{\prime}}$ be the disk defined above containing $\sigma_{m}\left(z_{0}\right)$. Then $D_{x^{\prime}}$ is contained in $V$ and it also intersects a point $w^{\prime}$ in $\widetilde{\alpha}_{w}$ (cf Figure 3). So $d\left(\sigma_{m}\left(z_{0}\right), w^{\prime}\right) \leq d\left(\sigma_{m}\left(z_{0}\right), x^{\prime}\right)+d\left(x^{\prime}, w^{\prime}\right) \leq \epsilon+\epsilon$. Hence $2 \epsilon<d\left(z_{0}, w\right) \leq$ $d\left(z_{0}, w^{\prime}\right)=d\left(p_{i, k}\left(z_{0}\right), p_{i, k}\left(w^{\prime}\right)\right)=d\left(p_{i, k}\left(\sigma_{m}\left(z_{0}\right)\right), p_{i, k}\left(w^{\prime}\right)\right) \leq d\left(\sigma_{m}\left(z_{0}\right), w^{\prime}\right) \leq 2 \epsilon$ (here the first equality follows from the property that $\tilde{\alpha}$ is a distance minimizing curve), giving a contradiction.

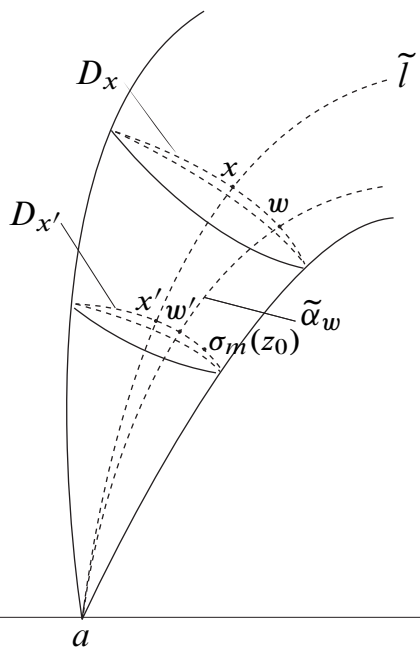

Figure 3

Let $R$ be a fixed number bigger than the number $R(\epsilon)$ provided in Proposition 4.5 and also bigger than the upper bound provided by Corollary 5.6 for the lengths of $I$-fibers of $X_{i}$ (for each of $\left.i=1,2\right)$. Consider $N_{\left(R, X_{i}\right)}\left(Z_{i, j_{k}}\right)$, the $R$-collared neighborhood 
of $Z_{i, j_{k}}$ in $X_{i}$. It is a convex 3-submanifold in $X_{i} \subset \mathbb{H}^{3}$ (thus is simply connected) and is invariant under the action of $\Gamma_{i, j_{k}}$. We let $A N_{\left(R, X_{i}\right)}\left(K_{i, k}\right)$ denote the quotient space $N_{\left(R, X_{i}\right)}\left(Z_{i, j_{k}}\right) / \Gamma_{i, j_{k}}$, and call it the abstract $R$-collared neighborhood of $K_{i, k}$ with respect to $X_{i}$. Similarly we can define the truncated version of $N_{\left(R, X_{i}\right)}\left(Z_{i, j_{k}}\right)$ and the truncated version of $A N_{\left(R, X_{i}\right)}\left(K_{i, k}\right)$, denoted by $\left(N_{\left(R, X_{i}\right)}\left(Z_{i, j_{k}}\right)\right)^{-}$and $\left(A N_{\left(R, X_{i}\right)}\left(K_{i, k}\right)\right)^{-}$respectively. It follows from Lemma 6.6 that $\left(A N_{R, X_{i}}\left(K_{i, k}\right)\right)^{-}$ is compact. We can extend $g_{i, k}: K_{i, k} \rightarrow Y_{i}$ to a map, which we still denote $g_{i, k}$, from $A N_{\left(R, X_{i}\right)}\left(K_{i, k}\right)$ to $Y_{i}$

By construction, $N_{\left(R, X_{i}\right)}\left(Z_{i, j_{k}}\right)$ contains all the $I$-fibers of $X_{i}$ which meet $Z_{i, j_{k}}$. Let $Z_{i, j_{k}}^{\prime}$ be the sub- $I$-bundle of $X_{i}$ consisting of all the $I$-fibers of $X_{i}$ which meet $Z_{i, j_{k}}$. It is easy to see that $Z_{i, j_{k}}^{\prime}$ is a manifold. The manifold $Z_{i, j_{k}}^{\prime}$ is also invariant under the action of $\Gamma_{i, j_{k}}$ since $Z_{i, j_{k}}$ is invariant under the action of $\Gamma_{i, j_{k}}$ and since the action of $\Gamma_{i, j_{k}} \subset \Gamma_{i}$ on $X_{i}$ sends fibers to fibers. Hence $Z_{i, j_{k}}^{\prime} / \Gamma_{i, j_{k}}=F_{i, k} \times I$ for some surface $F_{i, k}$ (which is non-compact), with the induced $I$-fiber structure. From the inclusions $K_{i, k} \subset F_{i, k} \times I \subset A N_{\left(R, X_{i}\right)}\left(K_{i, k}\right)$ and from the fact that the inclusion map $K_{i, k} \subset A N_{\left(R, X_{i}\right)}\left(K_{i, k}\right)$ induces an isomorphism on the fundamental groups, we see that the inclusion map $F_{i, k} \times I \subset A N_{\left(R, X_{i}\right)}\left(K_{i, k}\right)$ induces a surjective homomorphism on the fundamental groups.

Note that $\partial F_{i, k} \times I$ is precisely the frontier of $F_{i, k} \times I$ in $A N_{\left(R, X_{i}\right)}\left(K_{i, k}\right)$. Each component of $\partial F_{i, k} \times I$ is either an annulus or a strip, where a strip means $\mathbb{R} \times I$.

Lemma 6.7 Let $A$ be an annulus component of $\partial F_{i, k} \times I$. Then:

(1) $A$ divides $A N_{\left(R, X_{i}\right)}\left(K_{i, k}\right)$ into two components $B_{1}$ and $B_{2}$.

(2) Suppose $B_{1}$ is the component whose interior is disjoint from $F_{i, k} \times I$. Then either $B_{1}=D \times I$, where $D$ is a disk, such that $A=\partial D \times I$; or $B_{1}=S^{1} \times D$, where $D$ is a disk, such that $A=I \times S^{1}$, where $I$ is an interval contained in $\partial D$.

Proof Since $F_{i, k} \times I$ is a submanifold of $A N_{\left(R, X_{i}\right)}\left(K_{i, k}\right)$ and carries the fundamental group of $A N_{\left(R, X_{i}\right)}\left(K_{i, k}\right)$, it follows that $A$ is separating in $A N_{\left(R, X_{i}\right)}\left(K_{i, k}\right)$, ie we have (1). Part (2) also follows easily.

Similarly we have:

Lemma 6.8 Let $E$ be a strip component of $\partial F_{i, k} \times I$. Then:

(1) $E$ divides $A N_{\left(R, X_{i}\right)}\left(K_{i, k}\right)$ into two components $B_{1}$ and $B_{2}$.

(2) Suppose $B_{1}$ is the component whose interior is disjoint from $F_{i, k} \times I$. Then $B_{1}=\mathbb{R} \times D$, where $D$ is a disk, such that $E=\mathbb{R} \times I$, where $I$ is an interval contained in $\partial D$. 
It follows from Lemmas 6.7 and 6.8 that the $I$ bundle structure of $F_{i, k} \times I$ can be extended to one on $A N_{\left(R, X_{i}\right)}\left(K_{i, k}\right)$ in an obvious way.

Similarly one can obtain corresponding results in the truncated setting. Namely $\left(A N_{\left(R, X_{i}\right)}\left(K_{i, k}\right)\right)^{-}$has a sub-manifold of the form $F_{i, k}^{-} \times I$ which carries the fundamental group of $\left(A N_{\left(R, X_{i}\right)}\left(K_{i, k}\right)\right)^{-}$, and the $I$-bundle structure of $F_{i, k}^{-} \times I$ can be extended to one on $\left(A N_{\left(R, X_{i}\right)}\left(K_{i, k}\right)\right)^{-}$in an obvious way, such that the parabolic boundary of $\left(A N_{\left(R, X_{i}\right)}\left(K_{i, k}\right)\right)^{-}$consists of $I$-fibers. Note that the $I$-fiber structure may not agree with the original $I$-fiber structure on $X_{i} / \Gamma_{i, j}$.

Since $\left(A N_{\left(R, X_{i}\right)}\left(K_{i, k}\right)\right)^{-}$is compact for each $i=1,2, k=1, \ldots, q$, and since the horoball region of $A N_{\left(R, X_{i}\right)}\left(K_{i, k}\right)$ has a standard shape, we may assume, up to replacing the cusp $C$ of $M$ by a smaller one, that $g_{i, k}^{-1}\left(\mathcal{C}_{i}\right) \cap\left(A N_{\left(R, X_{i}\right)}\left(K_{i, k}\right)\right)^{-}=$ $\partial_{p}\left(A N_{\left(R, X_{i}\right)}\left(K_{i, k}\right)\right)^{-}\left(\right.$where $\mathcal{C}_{i}$ is the cusp part of $\left.Y_{i}\right)$ for each of $i=1,2, k=$ $1, \ldots, q$.

We let $A N_{\left(R, X_{i}\right)}\left(K_{i}\right)$ denote the disjoint union of $\left\{A N_{\left(R, X_{i}\right)}\left(K_{i, k}\right) ; k=1, \ldots, q\right\}$, and let $g_{i}: A N_{\left(R, X_{i}\right)}\left(K_{i}\right) \rightarrow Y_{i}$, extending the local isometries $g_{i, k}$. For later use, we record the following corollary.

Corollary 6.9 Suppose that the local isometry

$$
g_{i}:\left(A N_{\left(R, X_{i}\right)}\left(K_{i}\right)\right)^{-}=\amalg_{k}\left(A N_{\left(R, X_{i}\right)}\left(K_{i, k}\right)\right)^{-} \rightarrow Y_{i}^{-}
$$

lifts to an embedding in a finite cover $\breve{Y}_{i}^{-}$of $Y_{i}^{-}$. Then the $I$-bundle structure on $\breve{Y}_{i}^{-}$ can be adjusted to one so that the image of $\left(A N_{\left(R, X_{i}\right)}\left(K_{i}\right)\right)^{-}$is a sub-I-bundle in $\breve{Y}_{i}^{-}$.

Let $F_{X_{i}}\left(N_{\left(R, X_{i}\right)}\left(Z_{i, j_{k}}\right)\right)$ denote the frontier of $N_{\left(R, X_{i}\right)}\left(Z_{i, j_{k}}\right)$ in $X_{i}$. If we define the frontier boundary $\partial_{f}\left(A N_{\left(R, X_{i}\right)}\left(K_{i, k}\right)\right)$ of $A N_{\left(R, X_{i}\right)}\left(K_{i, k}\right)$ to be

$$
F r_{X_{i}}\left(N_{\left(R, X_{i}\right)}\left(Z_{i, j_{k}}\right)\right) / \Gamma_{i, j_{k}}
$$

then $\partial_{f}\left(A N_{\left(R, X_{i}\right)}\left(K_{i, k}\right)\right)$ is topologically parallel to $\partial F_{i, k} \times I$ by Lemmas 6.7 and 6.8. Thus each component of $\partial_{f}\left(A N_{\left(R, X_{i}\right)}\left(K_{i, k}\right)\right)$ is either an annulus or a strip. A strip component must enter the cusp region of $A N_{\left(R, X_{i}\right)}\left(K_{i, k}\right)$. From the shape of the cusp region of $A N_{\left(R, X_{i}\right)}\left(K_{i, k}\right)$ and from Lemma 6.5, we see that the frontier boundary of $A N_{\left(R, X_{i}\right)}\left(K_{i}\right)$ has exactly $d$ strip components and that the frontier boundary of each component $A N_{\left(R, X_{i}\right)}\left(K_{i, k}\right)$ of $A N_{\left(R, X_{i}\right)}\left(K_{i}\right)$ has at least two strip components. We restate this fact in the following corollary for later use. 


\section{Corollary 6.10}

(1) $\partial_{f}\left(A N_{\left(R, X_{i}\right)}\left(K_{i, k}\right)\right)$ has at least two strip components for each $i=1,2$ and $k=1, \ldots, q$.

(2) $\partial_{f}\left(A N_{\left(R, X_{i}\right)}\left(K_{i}\right)\right)$ has exactly $d$ strip components for each $i=1,2$.

The following corollary follows easily from the convexity of $N_{\left(R, X_{i}\right)}\left(Z_{i, j_{k}}\right)$ and from the shape of the parabolic region of $N_{\left(R, X_{i}\right)}\left(Z_{i, j_{k}}\right)$.

Corollary 6.11 Every component of $F r_{X_{i}}\left(N_{\left(R, X_{i}\right)}\left(Z_{i, j_{k}}\right)\right)$ has its two ends contained in two different horoball components of $\mathcal{B}$ respectively.

We conclude with some remarks.

Remark 6.12 Results and notations in this section will still be valid if we replace the cusp $C$ by a smaller one.

Remark 6.13 In the construction of $K_{i, k}$ and its local isometry $g_{i, k}$ into $Y_{i}$ some choices were made (for instance the universal cover $Z_{i, j_{k}}$ in $X_{i}$ which is defined up to translation by elements of $\Gamma_{i}$ ). But, up to isometry, the construction is independent of all such choices; ie if $g_{i, k}^{\prime}: K_{i, k}^{\prime} \rightarrow Y_{i}$ is another result of this construction, then there is an isometry $\phi_{i, k}: K_{i, k} \rightarrow K_{i, k}^{\prime}$ such that $g_{i, k}=g_{i, k}^{\prime} \circ \phi_{i, k}$.

\section{Constructing $J_{i}$}

In Section 6, we constructed, for each $i=1,2$, the manifold $A N_{\left(R, X_{i}\right)}\left(K_{i}\right)$, which is the disjoint union of $\left\{A N_{\left(R, X_{i}\right)}\left(K_{i, k}\right) ; k=1, \ldots, q\right\}$, such that each component of $A N_{\left(R, X_{i}\right)}\left(K_{i}\right)$ is a metrically complete, convex, hyperbolic 3-manifold, and we defined a local isometry $g_{i}: A N_{\left(R, X_{i}\right)}\left(K_{i}\right) \rightarrow Y_{i}$. In this section we construct, for each $i=1,2$, a connected, metrically complete, convex, hyperbolic 3-manifold $J_{i}$ with a local isometry $g_{i}: J_{i} \rightarrow Y_{i}$, such that $J_{i}$ contains $A N_{\left(R, X_{i}\right)}\left(K_{i}\right)$ as a hyperbolic submanifold, and $J_{i} \backslash A N_{\left(R, X_{i}\right)}\left(K_{i}\right)$ is a compact 3-manifold $W_{i}$ (which may not be connected). Obviously we may assume that $q>1$, since otherwise we may simply take $J_{i}=A N_{\left(R, X_{i}\right)}\left(K_{i, 1}\right)$.

We continue to use the notations established in early sections. We have showed (Corollary 6.10) that $\partial_{f}\left(A N_{\left(R, X_{i}\right)}\left(K_{i, k}\right)\right)$ has at least two strip components for each $k=1, \ldots, q$ and that $\partial_{f}\left(A N_{\left(R, X_{i}\right)}\left(K_{i}\right)\right)$ has exactly $d$ strip components. Let $E_{i, k}$ be a fixed strip component of $\partial_{f} A N_{\left(R, X_{i}\right)}\left(K_{i, k}\right)$ for each fixed $i$ and $k$. Recall $p_{i, k}: N_{\left(R, X_{i}\right)}\left(Z_{i, j_{k}}\right) \rightarrow A N_{\left(R, X_{i}\right)}\left(K_{i, k}\right)$ is the universal covering map. Then each 
component of $p_{i, k}^{-1}\left(E_{i, k}\right) \subset F r_{X_{i}}\left(N_{\left(R, X_{i}\right)}\left(Z_{i, j_{k}}\right)\right)$ is a strip isometric to $E_{i, k}$ under the map $p_{i, k}$. Let $\widetilde{E}_{i, k}$ be a fixed component of $p_{i, k}^{-1}\left(E_{i, k}\right)$.

The required $J_{i}$ will be constructed by gluing components of $A N_{\left(R, X_{i}\right)}\left(K_{i}\right)$ with a compact 3-manifold $W_{i}$, along an attaching region in $\amalg E_{i, k}$. We shall construct the connecting manifold $W_{i}$ in $X_{i}$. The procedure is as follows: find a suitable translation of $N_{\left(R, X_{i}\right)}\left(Z_{i, j_{k}}\right)$ by an element $\tau_{i, k}$ of $\Gamma_{i}$, then take the convex hull of $\left\{\tau_{i, k}\left(N_{\left(R, X_{i}\right)}\left(Z_{i, j_{k}}\right)\right) ; k=1, \ldots, q\right\}$ in $X_{i}$. The added part in forming the convex hull is the manifold $W_{i}$, which will be shown to be compact, and the attaching region of $W_{i}$ with $\tau_{i, k}\left(N_{\left(R, X_{i}\right)}\left(Z_{i, j_{k}}\right)\right)$ is contained in $\tau_{i, k}\left(\widetilde{E}_{i, k}\right)$.

If such $W_{i}$ can be found, we can glue it with each $A N_{\left(R, X_{i}\right)}\left(K_{i, k}\right)$ along $E_{i, k}$ using the isometry $\tau_{i, k}\left(\widetilde{E}_{i, k}\right) \stackrel{\tau_{i, k}^{-1}}{\rightarrow} \widetilde{E}_{i, k} \stackrel{p_{i, k}}{\rightarrow} E_{i, k}$. The resulting manifold $J_{i}$ is a convex hyperbolic 3-manifold, with a local isometry into $Y_{i}$, extending the map $g_{i}: A N_{\left(R, X_{i}\right)}\left(K_{i}\right) \rightarrow Y_{i}$. It is easy to see that the hyperbolic structure in $A N_{\left(R, X_{i}\right)}\left(K_{i}\right)$ and the hyperbolic structure on $W_{i}$ match up along their gluing surfaces, forming a global hyperbolic structure for $J_{i}$.

We now give the construction of $W_{i}$, beginning with some well known facts. Let $\gamma$ be any hyperbolic element of $P S L_{2}(\mathbb{C})$. The axis of $\gamma$ is denoted $A_{\gamma}$. Let $a, a^{\prime}$ be the two limit points of $A_{\gamma}$, which are the two fixed points of $\gamma$ in $S_{\infty}^{2}$. Then for any point $x$ in $\overline{\mathbb{H}}^{3}$, the sequence $\gamma^{n}(x)$ approaches one of the points $a, a^{\prime}$, say $a$, as $n \rightarrow \infty$, and approaches $a^{\prime}$, as $n \rightarrow-\infty$. Thus for any fixed closed subset $W$ of $\overline{\mathbb{H}}^{3}$ which is disjoint from $a^{\prime}$, and for any fixed open neighborhood $U$ of $a$ in $\overline{\mathbb{H}}^{3}$ there is an integer $n$ such that $\gamma^{n}(W) \subset U$.

Lemma 7.1 For any open arc $\alpha$ in $\Lambda_{i}=\Lambda\left(\Gamma_{i}\right)$, there exists a hyperbolic element $\gamma$ of $\Gamma_{i}$ such that the two limit points of $A_{\gamma}$ are contained in $\alpha$.

Proof Since fixed points of hyperbolic elements of $\Gamma_{i}$ are dense in $\Lambda_{i}$, there is a hyperbolic element $\delta$ in $\Gamma_{i}$ with at least one of its two fixed points contained in $\alpha$. Now take a hyperbolic element $\eta$ of $\Gamma_{i}$ such that the limit points of $A_{\eta}$ are disjoint from the limit points of $A_{\delta}$. By the notes given proceeding the lemma, there is an integer $n$ such that the two limit points of $\delta^{n}\left(A_{\eta}\right)$ are both contained in $\alpha$. Let $\gamma=\delta^{n} \eta \delta^{-n}$, then $A_{\gamma}=\delta^{n}\left(A_{\eta}\right)$.

Each strip $\widetilde{E}_{i, k}$ (defined earlier in this section) has exactly two limit points in $\Lambda_{i}$ and each of them is a parabolic fixed point of $\Gamma_{i}$ (the two parabolic fixed points are distinct because of the convexity of $\left.N_{\left(R, X_{i}\right)}\left(Z_{i, j_{k}}\right)\right)$. Note that $\widetilde{E}_{i, k}$ separates $X_{i}$ into two 
parts, one of which contains $N_{\left(R, X_{i}\right)}\left(Z_{i, j_{k}}\right)$. Let $U_{i, k}$ be the part whose interior is disjoint from $N_{\left(R, X_{i}\right)}\left(Z_{i, j_{k}}\right)$. Let $\alpha_{i, k}$ be the limit set of $U_{i, k}$.

Lemma 7.2 After translations by suitable elements of $\Gamma_{i}$, we may assume that $N_{\left(R, X_{i}\right)}\left(Z_{i, j_{2}}\right)$ is contained in the interior of $U_{i, 1}$ and that $N_{\left(R, X_{i}\right)}\left(Z_{i, j_{1}}\right)$ is contained in the interior of $U_{i, 2}$

Proof Let $\bar{X}_{i}$ denote the closure of $X_{i}$ in $\overline{\mathbb{H}}^{3}$. Note that $N_{\left(R, X_{i}\right)}\left(Z_{i, j_{k}}\right)$ has the same limit set as $Z_{i, j_{k}}$. Let $\gamma$ be a hyperbolic element of $\Gamma_{i}$ whose axis has a limit point, $a$, disjoint from the limit points of $N_{\left(R, X_{i}\right)}\left(Z_{i, j_{2}}\right)$. Then by the notes given in the paragraph proceeding Lemma 7.1, we may move $N_{\left(R, X_{i}\right)}\left(Z_{i, j_{2}}\right)$ by a power of $\gamma$ into a small open neighborhood of $a^{\prime}$ (which is the other limit point of $A_{\gamma}$ ) in $\bar{X}_{i}$. In particular, we may assume that the limit set of this translate does not contain the limit set of $U_{i, 1}$. Then, applying Lemma 7.1, there is an element in $\Gamma_{i}$ which translates $N_{\left(R, X_{i}\right)}\left(Z_{i, j_{2}}\right)$ into $U_{i, 1}$.

Thus we may assume that $N_{\left(R, X_{i}\right)}\left(Z_{i, j_{2}}\right)$ is contained in the interior of $U_{i, 1}$. If $N_{\left(R, X_{i}\right)}\left(Z_{i, j_{1}}\right)$ is contained in the interior of $U_{i, 2}$ already, then we are done. So suppose not. Then $U_{i, 2}$ is contained in the interior of $U_{i, 1}$. Let $\gamma$ be a hyperbolic element of $\Gamma_{i}$ such that the two limit points of $A_{\gamma}$ are contained in the interior of the $\operatorname{arc} \alpha_{i, 2}$; such an element exists by Lemma 7.1. Then, after replacing $N_{\left(R, X_{i}\right)}\left(Z_{i, j_{2}}\right)$ by its translate under a suitably high power of $\gamma$, one may check that the conclusion of the lemma is satisfied.

By Lemma 7.2, $\widetilde{E}_{i, 1}$ and $\widetilde{E}_{i, 2}$ co-bound a connected submanifold of $X_{i}, V_{1}$, whose interior is disjoint from both $N_{\left(R, X_{i}\right)}\left(Z_{i, j_{1}}\right)$ and $N_{\left(R, X_{i}\right)}\left(Z_{i, j_{2}}\right)$. The limit set of $V_{1}$ consists of two disjoint arcs in $\Lambda_{i}$. Now if $q>2$, then by a method similar to the proof of Lemma 7.2, we may assume, up to translation by a hyperbolic element of $\Gamma_{i}$, that $N_{\left(R, X_{i}\right)}\left(Z_{i, j_{3}}\right)$ is in in the interior of $V_{1}$, and that both $N_{\left(R, X_{i}\right)}\left(Z_{i, j_{1}}\right)$ and $N_{\left(R, X_{i}\right)}\left(Z_{i, j_{2}}\right)$ are contained in the interior of $U_{i, 3}$. In other words, the three strips $\widetilde{E}_{i, 1}, \widetilde{E}_{i, 2}$ and $\widetilde{E}_{i, 3}$ co-bound a connected submanifold $V_{2}$ in $X_{i}$ such that the interior of $V_{2}$ is disjoint from $N_{\left(R, X_{i}\right)}\left(Z_{i, j_{1}}\right), N_{\left(R, X_{i}\right)}\left(Z_{i, j_{2}}\right)$ and $N_{\left(R, X_{i}\right)}\left(Z_{i, j_{3}}\right)$.

By a simple induction, we may assume that $N_{\left(R, X_{i}\right)}\left(Z_{i, j_{k}}\right), k=1, \ldots, q$, are located in $X_{i}$ in such way that the $q$ strips $\widetilde{E}_{i, k}, k=1, \ldots, q$, co-bound a connected submanifold $V$ of $X_{i}$ whose interior is disjoint from $N_{\left(R, X_{i}\right)}\left(Z_{i, j_{k}}\right), k=1, \ldots, q$. Now we take the convex hull of the set $\left\{N_{\left(R, X_{i}\right)}\left(Z_{i, j_{k}}\right), k=1, \ldots, q\right\}$ in $X_{i}$, and let $Z_{i}$ be the resulting convex manifold. Let $W_{i}$ be the complement of the interior of $N_{\left(R, X_{i}\right)}\left(Z_{i, j_{k}}\right), k=$ $1, \ldots, q$, in $Z_{i}$. Then, by Proposition $4.6, W_{i}$ is a compact submanifold of $X_{i}$. This 
$W_{i}$ is the desired connecting manifold. The attaching region in $\partial W$, to be glued to $E_{i, k}$, is $W_{i} \cap \widetilde{E}_{i, k}$.

We still use $g_{i}$ to denote the local isometry $J_{i} \rightarrow Y_{i}$. Since $W_{i}$ is compact, there exists a cusp $C^{\prime}$ of $M$, smaller than or equal to $C$, such that $g_{i}\left(W_{i}\right)$ is disjoint from the corresponding cusp region $\mathcal{C}^{\prime}{ }_{i}$ of $Y_{i}$. We may assume that the cusp $C$ itself already satisfies this condition. Under this assumption, $W_{i}$ is disjoint from $A N_{\left(R, X_{i}\right)}\left(K_{i}\right) \backslash$ $\left(A N_{\left(R, X_{i}\right)}\left(K_{i}\right)\right)^{-}$, and the components of $\left(A N_{\left(R, X_{i}\right)}\left(K_{i}\right)\right)^{-}$are connected together by $W_{i}$ along the frontier boundary of $A N_{\left(R, X_{i}\right)}\left(K_{i}\right)$, forming a connected compact manifold which we denote by $J_{i}^{-}$. The parabolic boundary $\partial_{p} J_{i}^{-}$is defined to be the parabolic boundary of $\left(A N_{\left(R, X_{i}\right)}\left(K_{i}\right)\right)^{-}$. Then $g_{i} \mid:\left(J_{i}^{-}, \partial_{p} J_{i}^{-}\right) \rightarrow\left(Y_{i}^{-}, \partial_{p} Y_{i}^{-}\right)$is a proper map of pairs.

Each component of $\partial_{p} J_{i}^{-}$can be isometrically embedded in $\partial B_{\infty}$ as a Euclidean parallelogram. The convex hull of such a parallelogram is a convex 3-ball in $B_{\infty}$ lying vertically above the parallelogram. We let $\widehat{J}_{i}$ denote the manifold obtained by capping off each of its parabolic boundary component by a convex 3-ball as just described. Then $\widehat{J}_{i}$ is a connected, compact, convex 3 -manifold with a local isometry (which we still denote by $g_{i}$ ) into $Y_{i}$.

\section{Constructing $C_{n}\left(J_{i}^{-}\right)$}

From Sections 5, 6 and 7, we have the following setting: for each $i=1,2, f_{i}: Y_{i}=$ $S_{i} \times I \rightarrow M$ is a local isometry; $f_{i} \mid:\left(Y_{i}^{-}=S_{i}^{-} \times I, \partial_{p} Y_{i}^{-}=\partial S_{i}^{-} \times I\right) \rightarrow(M, \partial M)$ is a proper map; $f_{i} \mid: \partial S_{i}^{-} \rightarrow \partial M$ is an embedding; $\partial S_{i}^{-}$has $n_{i}$ components $\left\{\beta_{i, j}, j=\right.$ $\left.1, \ldots, n_{i}\right\}$ with induced orientation; $\Delta$ is the geometric intersection number between $f_{1}\left(\beta_{1,1}\right)$ and $f_{2}\left(\beta_{2,1}\right)$; there are $d=\Delta n_{1} n_{2}$ intersection points $\left\{t_{1}, \ldots, t_{d}\right\}$ between $f_{1}\left(\partial S_{1}^{-}\right)$and $f_{2}\left(\partial S_{2}^{-}\right)$in $\partial M ;\left\{t_{i, j, k}, j=1, \ldots, n_{i}, k=1, \ldots, d_{i}\right\}$ are the points in $f_{i}^{-1}\left\{t_{1}, \ldots, t_{d}\right\}$, where $d_{i}=\Delta n_{i_{*}}$, indexed so that $\left\{t_{i, j, k}, k=1, \ldots, d_{i}\right\}$ are contained successively in the component $\beta_{i, j}$ (following the orientation of $\beta_{i, j}$ ) for each $j=$ $1, \ldots, n_{i} ; K_{i}$ is the disjoint union of the "intersection manifolds" $\left\{K_{i, j}, j=1, \ldots, q\right\}$; the manifold $A N_{\left(R, X_{i}\right)}\left(K_{i}\right)=\Pi A N_{\left(R, X_{i}\right)}\left(K_{i, k}\right)$ is the abstract $R$-collared neighborhood of $K_{i}$ with respect to $X_{i}$; each component $A N_{\left(R, X_{i}\right)}\left(K_{i, k}\right)$ is a metrically complete, convex, hyperbolic 3-manifold with a local isometry $g_{i, k}: A N_{\left(R, X_{i}\right)}\left(K_{i, k}\right) \rightarrow$ $Y_{i}$; the restriction $g_{i, k} \mid:\left(\left(A N_{\left(R, X_{i}\right)}\left(K_{i, k}\right)\right)^{-}, \partial_{p}\left(A N_{\left(R, X_{i}\right)}\left(K_{i, k}\right)\right)^{-}\right) \rightarrow\left(Y_{i}^{-}, \partial_{p} Y_{i}^{-}\right)$ is a proper map; $g_{i}: A N_{\left(R, X_{i}\right)}\left(K_{i}\right) \rightarrow Y_{i}$ is the local isometry with $\left.g_{i}\right|_{A N_{\left(R, X_{i}\right)}\left(K_{i, k}\right)}=$ $g_{i, k} ; J_{i}$ is a metrically complete convex (thus connected) hyperbolic 3 -manifold with local isometry $g_{i}: J_{i} \rightarrow Y_{i} ; g_{i} \mid:\left(J_{i}^{-}, \partial_{p} J_{i}^{-}\right) \rightarrow\left(Y_{i}^{-}, \partial_{p} Y_{i}^{-}\right)$is a proper map; $J_{i}$ contains $A N_{\left(R, X_{i}\right)}\left(K_{i}\right)$ as a submanifold; and $\partial_{p} J_{i}^{-}=\partial_{p}\left(A N_{\left(R, X_{i}\right)}\left(K_{i}\right)\right)^{-}$. 
Also recall that there are exactly $d$ components in $\partial_{p} J_{i}^{-}=\partial_{p}\left(A N_{\left(R, X_{i}\right)}\left(K_{i}\right)\right)^{-}$, one each associated to the points $t_{1}, \ldots, t_{d}$ respectively. Let $D_{i, j, k}, j=1, \ldots, n_{i}, k=$ $1, \ldots, d_{i}$ denote the components of $\partial_{p} J_{i}^{-}$and let $b_{i, j, k}$ be the topological center point of $D_{i, j, k}$, indexed so that $g_{i}\left(b_{i, j, k}\right)=t_{i, j, k}$.

The purpose of this section is to construct, for each sufficiently large integer $n$, a connected, compact, convex, hyperbolic 3-manifold $C_{n}\left(J_{i}^{-}\right)$, with a local isometry into $Y_{i}$, such that $C_{n}\left(J_{i}^{-}\right)$contains $J_{i}^{-}$as a hyperbolic submanifold. $C_{n}\left(J_{i}^{-}\right)$is obtained by gluing together $J_{i}^{-}$with $n_{i}$ "multi-l-handles" $H_{i, j}^{n}, j=1, \ldots, n_{i}$, along the attaching region $\partial_{p} J_{i}^{-}$(see Figure 4 for a preview). A more precise description of $C_{n}\left(J_{i}^{-}\right)$will be clear after its construction. The needed properties of $C_{n}\left(J_{i}^{-}\right)$will be described in later sections.

Now we proceed to construct the multi- 1 -handle $H_{i, j}^{n}$ for each fixed $i \in\{1,2\}$ and each fixed $j \in\left\{1, \ldots, n_{i}\right\}$. Let $c_{i, j}$ be a fixed component of $p^{-1}\left(f_{i}\left(\beta_{i, j}\right)\right)$ in the horizontal horosphere $\partial B_{\infty}$. The transitivity of the action of $\Gamma$ implies that there is element $\delta_{i, j} \in \Gamma$ such that $\delta_{i, j}\left(\tilde{S}_{i}\right)$ contains $c_{i, j}$. By Corollary 5.4, $\delta_{i, j}\left(X_{i}\right) \cap \partial B_{\infty}$ is a strip in $\partial B_{\infty}$ between two parallel Euclidean lines, which contains $c_{i, j}$ as its (topological) center line. Let $E_{i, j}$ denote this strip.

Along $c_{i, j}$, we index the set of points $p^{-1}\left(\left\{f_{i}\left(t_{i, j, k}\right), k=1, \ldots, d_{i}\right\}\right)$ as $a_{i, j, k, m}$, $k=1, \ldots, d_{i}, m \in \mathbb{Z}$, such that:

(1) for each fixed $m$, the points $\left\{a_{i, j, k, m}, k=1, \ldots, d_{i}\right\}$ appear consecutively along the line $c_{i, j}$ following the orientation of $c_{i, j}$ (which is induced from that of $\beta_{i, j}$ );

(2) the point $a_{i, j, d_{i}, m}$ is followed immediately by the point $a_{i, j, 1, m+1}$, for every $m$;

(3) $p\left(a_{i, j, k, m}\right)=f_{i}\left(t_{i, j, k}\right)$, for all $k=1, \ldots, d_{i}$ and $m \in \mathbb{Z}$.

For an arbitrary (fixed) sufficiently large integer $n>0$, consider the following $d_{i}$ points on $c_{i, j}: a_{i, j, 1,0}, a_{i, j, 2, n}, a_{i, j, 3,2 n}, \ldots, a_{i, j, d_{i},\left(d_{i}-1\right) n}$.

Again by transitivity of the action of $\Gamma$, there are elements $\gamma_{i, j, 1}, \gamma_{i, j, 2}, \ldots, \gamma_{i, j, d_{i}} \in \Gamma$ such that $\gamma_{i, j, 1}\left(\tilde{S}_{i_{*}}\right), \gamma_{i, j, 2}\left(\tilde{S}_{i_{*}}\right), \ldots, \gamma_{i, j, d_{i}}\left(\tilde{S}_{i_{*}}\right)$ contain the points $a_{i, j, 1,0}, a_{i, j, 2, n}$, $\ldots, a_{i, j, d_{i},\left(d_{i}-1\right) n}$ respectively. Consider the corresponding translations of:

$$
X_{i_{*}}: \gamma_{i, j, 1}\left(X_{i_{*}}\right), \gamma_{i, j, 2}\left(X_{i_{*}}\right), \ldots, \gamma_{i, j, d_{i}}\left(X_{i_{*}}\right)
$$

Each of $\delta_{i, j}\left(X_{i}\right) \cap \gamma_{i, j, 1}\left(X_{i_{*}}\right), \delta_{i, j}\left(X_{i}\right) \cap \gamma_{i, j, 2}\left(X_{i_{*}}\right), \ldots, \delta_{i, j}\left(X_{i}\right) \cap \gamma_{i, j, d_{i}}\left(X_{i_{*}}\right)$ is a translation of some component in $\left\{Z_{i, j_{1}}, \ldots, Z_{i, j_{q}}\right\}$.

Let $Z_{1}, \ldots, Z_{d_{i}}$ denote $\delta_{i, j}\left(X_{i}\right) \cap \gamma_{i, j, 1}\left(X_{i_{*}}\right), \ldots, \delta_{i, j}\left(X_{i}\right) \cap \gamma_{i, j, d_{i}}\left(X_{i_{*}}\right)$ respectively, and let $N_{R}\left(Z_{i}\right)=N_{\left(R, \delta_{i, j}\left(X_{i}\right)\right)}\left(Z_{i}\right)$. Each of $N_{R}\left(Z_{1}\right), \ldots, N_{R}\left(Z_{d_{i}}\right)$ is a translation of some component in $\left\{N_{\left(R, X_{i}\right)}\left(Z_{i, j_{1}}\right), \ldots, N_{\left(R, X_{i}\right)}\left(Z_{i, j_{q}}\right)\right\}$. 


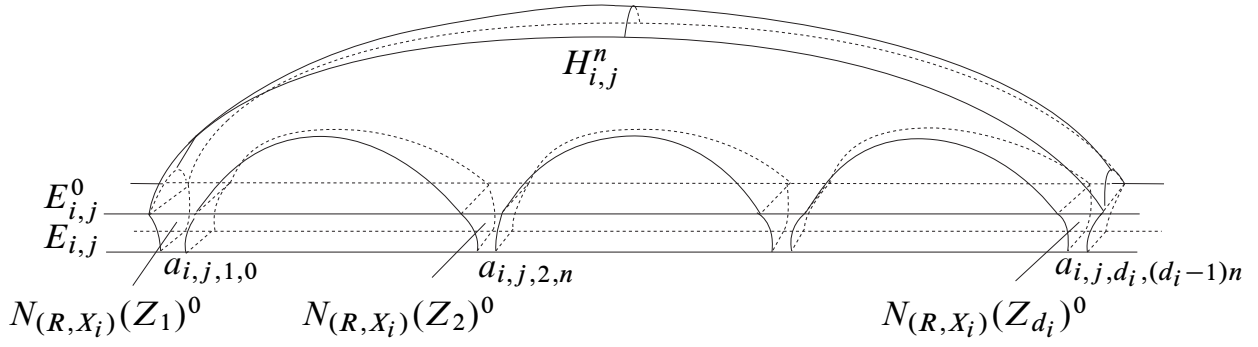

Figure 4

Let $B_{\infty}^{0}$ be a fixed horoball based at $\infty$ which is a little smaller than $B_{\infty}$, ie its boundary $\partial B_{\infty}^{0}$ is a little higher than $\partial B_{\infty}$. Let $E_{i, j}^{0}=\delta_{i, j}\left(X_{i}\right) \cap \partial B_{\infty}^{0}$, and let $N_{R}\left(Z_{1}\right)^{0}, \ldots, N_{R}\left(Z_{d_{i}}\right)^{0}$ be the part of $N_{R}\left(Z_{1}\right), \ldots, N_{R}\left(Z_{d_{i}}\right)$ between the two horizontal planes $\partial B_{\infty}^{0}$ and $\partial B_{\infty}$.

Then $N_{R}\left(Z_{1}\right)^{0} \cap \partial B_{\infty}, N_{R}\left(Z_{2}\right)^{0} \cap \partial B_{\infty}, \ldots, N_{R}\left(Z_{d_{i}}\right)^{0} \cap \partial B_{\infty}$ are Euclidean parallelograms contained in $E_{i, j}$, containing the points $a_{i, j, 1,0}, a_{i, j, 2, n}, \ldots, a_{i, j, d_{i},\left(d_{i}-1\right) n}$ as their topological center points, respectively, and they are isometric to $D_{i, j, k}, k=$ $1,2 \ldots, d_{i}$, respectively.

As $n$ is sufficiently large, $N_{R}\left(Z_{1}\right)^{0}, \ldots, N_{R}\left(Z_{d_{i}}\right)^{0}$ are mutually far apart from each other. We now take the convex hull of the set $\left\{N_{R}\left(Z_{1}\right)^{0}, \ldots, N_{R}\left(Z_{d_{i}}\right)^{0}\right\}$ in $\mathbb{H}^{3}$ and let $H_{i, j}^{n}$ be the resulting convex manifold. Obviously $H_{i, j}^{n}$ is contained in $\delta_{i, j}\left(X_{i}\right) \cap B_{\infty}$.

Let $U_{i, j}$ be the part of $\delta_{i, j}\left(X_{i}\right)$ between $E_{i, j}^{0}$ and $E_{i, j}$.

Then $N_{R}\left(Z_{1}\right)^{0}, \ldots, N_{R}\left(Z_{d_{i}}\right)^{0}$ are all contained in $U_{i, j}$, far apart from each other. We now show:

Lemma 8.1 If $n$ is sufficiently large, then:

$$
H_{i, j}^{n} \cap U_{i, j}=\left\{N_{R}\left(Z_{1}\right)^{0}, \ldots, N_{R}\left(Z_{d_{i}}\right)^{0}\right\}
$$

Proof Let $F_{k}$ be the frontier of $N_{R}\left(Z_{k}\right)^{0}$ in $U_{i, j}, 1 \leq k \leq d_{i}$. Then $F_{k}$ is contained in $\partial\left(N_{R}\left(\gamma_{i, j, k}\left(X_{i_{*}}\right)\right)\right)$. Since $N_{R}\left(\gamma_{i, j, k}\left(X_{i_{*}}\right)\right)$ is strictly convex and since $\partial N_{R}\left(\left(\gamma_{i, j, k}\left(X_{i_{*}}\right)\right)\right)$ is smooth, then for any point $x \in F_{k}$, there is a unique geodesic plane $P_{x}$ in $\mathbb{H}^{3}$ such that $P_{x} \cap N_{R}\left(\gamma_{i, j, k}\left(X_{i_{*}}\right)\right)=x$. Obviously $P_{x}$ is not a vertical plane. Thus $P_{x} \cap \partial B_{\infty}$ is a Euclidean circle in $\partial B_{\infty}$ with finite Euclidean diameter $a_{x}$. Since $F_{k}$ is compact, the set of numbers $\left\{a_{x} ; x \in F_{k}\right\}$ has a finite maximal value $a_{k}$. Let $a=\max \left\{a_{1}, \ldots a_{d_{i}}\right\}$, and let $c$ be the maximal Euclidean diameter of the parallelograms $\left\{N_{R}\left(Z_{1}\right)^{0} \cap E_{i, j}^{0}, \ldots, N_{R}\left(Z_{d_{i}}\right)^{0} \cap E_{i, j}^{0}\right\}$. By taking $n$ large enough, we can ensure that $N_{R}\left(Z_{1}\right)^{0} \cap E_{i, j}^{0}, \ldots, N_{R}\left(Z_{d_{i}}\right)^{0} \cap E_{i, j}^{0}$ are mutually far apart from 
each other by Euclidean distance at least $a+c$. Then the convex hull will satisfy the condition $H_{i, j}^{n} \cap U_{i, j}=\left\{N_{R}\left(Z_{1}\right)^{0}, \ldots, N_{R}\left(Z_{d_{i}}\right)^{0}\right\}$.

The manifold $H_{i, j}^{n}$ provided by Lemma 8.1 is the multi-1-handle we were seeking. (Figure 4 gives an illustration of $H_{i, j}^{n}$ when $d_{i}=4$ ). We may assume the choice of $n$ works in constructing all the multi- 1 -handles $H_{i, j}^{n}, j=1, \ldots, n_{i}, i=1,2$.

We now glue the multi- 1 -handles $H_{i, j}^{n}, j=1, \ldots, n_{i}$, to $J_{i}^{-}$along $D_{i, j, k}, k=$ $1, \ldots, d_{i}, j=1, \ldots, n_{i}$, (the gluing isometry should be clear). By our explicit construction, one can see that the hyperbolic structure on $H_{i, j}^{n}$ and the hyperbolic structure on $J_{i}^{-}$match up after the gluing, forming a global (convex) hyperbolic structure. Thus we obtain a compact, convex 3-manifold $C_{n}\left(J_{i}^{-}\right)$. We also have a local isometry $g_{i}: C_{n}\left(J_{i}^{-}\right) \rightarrow Y_{i}$, extending the local isometry $g_{i}: J_{i}^{-} \rightarrow Y_{i}$.

\section{Strong separability in the free group}

In this section, we present our main group theoretical result, (Theorem 9.1), which, together with the techniques used in its proof, will have crucial applications in this paper.

Let $S^{-}$be a connected, compact, orientable surface with genus $g$ and with $b>0$ boundary components. Fix a point $s$ in $S^{-}$as the base point, and let $F=\pi_{1}\left(S^{-}, s\right)$. Then $F$ is a free group. We may choose a free basis of $F$,

$$
a_{1}, b_{1}, a_{2}, b_{2}, \ldots, a_{g}, b_{g}, x_{1}, \ldots, x_{b-1},
$$

such that

$$
x_{1}, x_{2}, \ldots, x_{b-1}, x_{b}=\left[a_{1}, b_{1}\right]\left[a_{2}, b_{2}\right] \cdots\left[a_{g}, b_{g}\right] x_{1} x_{2} \cdots x_{b-1}
$$

are represented by embedded loops in $S^{-}$(based at $s$ ) which are freely isotopic to the $b$ boundary components of $S^{-}$respectively. An element $\gamma$ of $F$ is peripheral iff $\gamma$ is conjugate to some power of some $x_{i}$. We prove the following:

Theorem 9.1 Let $H \subset F$ be a finitely generated subgroup containing no nontrivial peripheral elements of $F$, and let $y_{1}, \ldots, y_{a} \in F-H$. Then there exists a subgroup $G$ of $F$, with $|F: G|=m<\infty$, such that $G$ contains $H$ but does not contain any elements of $\left\{y_{1}, \ldots, y_{a}, x_{i}, x_{i}^{2}, \ldots, x_{i}^{m-1}: i=1, \ldots, b\right\}$.

In particular, the subgroup $H$ is separable; indeed, by M Hall's Theorem, every finitely generated subgroup of $F$ is separable. However, Theorem 9.1 gives much more 
information about $G$, since the number of elements to be separated is tied up with the index of the subgroup $G$ in $F$.

As an aside, we record a topological consequence of Theorem 9.1, which may be of independent interest.

Corollary 9.2 Let $f: \alpha \rightarrow S^{-}$be an immersion of a geodesic loop in a hyperbolic surface $S^{-}$with $b>0$ boundary components. Then $f$ lifts to an embedding in a finite cover $\widetilde{S}^{-} \rightarrow S^{-}$, such that $\widetilde{S}^{-}$has exactly $b$ boundary components.

The proof of Theorem 9.1 is based on a technique, due originally to Stallings and developed thoroughly in Kapovich and Myasnikov [11], of using folded graphs. We first need to recall some definitions, and refer to [11] for more details. Let $L$ be a free basis for a free group $F$, and let $L^{-1}$ be the set $\left.\left\{x^{-1} ; x \in L\right\}\right)$. An $L$-labeled directed graph is a graph such that each edge of the graph is oriented, ie with an initial vertex and a terminal vertex assigned, and is labeled with a unique element of $L$. Given an $L$-labeled directed graph $\mathcal{G}$, we form an $L \cup L^{-1}$-labeled graph $\widehat{\mathcal{G}}$ as follows: for each edge $e$ of $\mathcal{G}$ - say with label $x$, initial vertex $v_{1}$ and terminal vertex $v_{2}$-add a new edge, denoted $e^{-1}$, with label $x^{-1}$, initial vertex $v_{2}$ and terminal vertex $v_{1}$. The introduction of $\widehat{\mathcal{G}}$ is purely for technique convenience.

An $L$-labeled directed graph $\mathcal{G}$ is said to be $L$-regular if, for every vertex $v$ of $\mathcal{G}$ and every $x \in L \cup L^{-1}$, there is exactly one edge of $\widehat{\mathcal{G}}$ with initial vertex $v$ and with label $x$. An $L$-labeled directed graph $\mathcal{G}$ is called folded if there is no pair of distinct edges $e, e^{\prime}$ in $\widehat{\mathcal{G}}$ with the same initial vertex and the same label. Obviously a regular graph is folded.

If $\mathcal{G}$ is folded, then every reduced path (ie path containing no subpath of the form $\left.e, e^{-1}\right)$ in $\widehat{\mathcal{G}}$ determines a unique freely reduced word in $L \cup L^{-1}$, and thus a unique element of $F$. If we fix a vertex $v_{0} \in \mathcal{G}$, then the set of all elements of $F$ corresponding to the set of reduced loops in $\widehat{\mathcal{G}}$ based at $v_{0}$ is a subgroup of $F$, denoted $L\left(\mathcal{G}, v_{0}\right)$. A proof of the following lemma is contained in Kapovich and Myasnikov [11].

Lemma 9.3 If $\mathcal{G}$ is a finite and $L$-regular graph, then $L\left(\mathcal{G}, v_{0}\right)$ is a finite-index subgroup of $F$, and its index in $F$ is equal to the number of vertices in $\mathcal{G}$.

An example of an $L$ directed graph $\mathcal{G}_{0}$ is the wedge of $|L|$ circles each given some fixed orientation and labeled with the labels of $L$, one each. If we denote the vertex by $v_{0}$, then $L\left(\mathcal{G}_{0}, v_{0}\right)=F$. The point of Lemma 9.3 is that a graph $\mathcal{G}$ as given in the lemma is naturally a finite sheeted covering of the graph $\mathcal{G}_{0}$ with degree equal to the number of vertices of $\mathcal{G}$. 
We now proceed to prove Theorem 9.1. From now on in this section $L$ denotes the free basis of the free group $F=\pi_{1}\left(S^{-}, s\right)$ given at the beginning of this section. We may certainly assume that $F$ is not a cyclic group, and thus we have either $g>0$, or $g=0$ and $b>2$. Elements of $F$ will be considered as words in letters in $L \cup L^{-1}$. It follows directly from the proof of Hall's Theorem in [11] that there is a connected, finite, folded, $L$-labeled directed graph $\mathcal{G}_{0}$, with base vertex $v_{0}$, such that $L\left(\mathcal{G}_{0}, v_{0}\right)=H$, and the words $y_{1}, \ldots, y_{a}$ are representable by non-closed paths in $\widehat{\mathcal{G}}_{0}$ with the base vertex $v_{0}$ as their initial vertex. Also, no loop in $\widehat{\mathcal{G}}_{0}$ (based at any vertex) represents a non-zero power of any $x_{i}$, for otherwise $H$ would contain nontrivial peripheral elements. Note that $\mathcal{G}_{0}$ is the quotient of the minimal $H$-invariant subtree of the Cayley graph of $F$ with respect to the given generators.

We need some more definitions. Suppose that $\mathcal{G}$ is a finite, connected, $L$-labeled directed graph. For each $i=1, \ldots, b$, we call a path in $\widehat{\mathcal{G}}$ an $x_{i}$-path if it represents a subword of the word $x_{i}^{k}$ for some non-negative integer $k$. A single vertex of the graph is also considered as an $x_{i}$-path, corresponding to the empty subword of $x_{i}$. An $x_{i}$-path is called an $x_{i}$-loop if it is a loop representing the word $x_{i}^{k}$ for some positive integer $k$. An $x_{i}$-path is called maximal if it is not contained in any other $x_{i}$-path besides itself. Now suppose further that $\mathcal{G}$ is folded and $\widehat{\mathcal{G}}$ contains no $x_{i}$-loops, for all $i=1, \ldots, b$. Then for each $i=1, \ldots, b$, every $x_{i}$-path is contained in a unique maximal $x_{i}$-path with finite length (where the length of a path is the number of edges that the path contains). If $i<b$, then any maximal $x_{i}$-path is an embedded path, and any two different maximal $x_{i}$-paths are disjoint. For a maximal $x_{b}$-path, every oriented edge in the path appears only once in the path but the path may cross itself at some common vertices. Any two different maximal $x_{b}$-paths have disjoint oriented edges but may cross each other at some common vertices. It follows that there are only finitely many maximal $x_{i}$-paths in $\widehat{\mathcal{G}}$, which we denote by $C_{i, j}, i=1, \ldots, b$, $j=1, \ldots, m_{i}$. For a maximal $x_{i}$-path $C_{i, j}$, its initial (respectively terminal) vertex is missing an incoming (respectively outgoing) edge whose label is the predecessor (respectively successor) to the first (respectively the last) label of $C_{i, j}$, where $C_{i, j}$ is considered as a subword of the word $x_{i}^{k}$ (for some $k \geq 0$ ). We shall call these two missing labels the initial and terminal missing labels of $C_{i, j}$ respectively. Of course if $i<b$, then the initial or terminal missing label for every $C_{i, j}$ is always $x_{i}$. Note that for a maximal $x_{b}$-path $C_{b, j}$, if the first label of $C_{b, j}$ is the letter $a_{1}$, then the initial missing label of $C_{b, j}$ is the letter $x_{b-1}$ if $b>1$ or the latter $b_{g}^{-1}$ if $b=1$; and similarly if the last label of $C_{b, j}$ is the letter $b_{g}^{-1}$, then the terminal missing label of $C_{b, j}$ is the letter $x_{1}$ if $b>1$ or the letter $a_{1}$ if $b=1$.

Lemma 9.4 Let $\mathcal{G}$ be a finite, connected, $L$-labeled, directed graph such that $\mathcal{G}$ is folded and such that $\widehat{\mathcal{G}}$ contains no $x_{i}$-loops for any $i=1, \ldots, b$. Then $x \in L$ is the 
initial missing label of some maximal $x_{i}$-path $C_{i, j}$ if and only if $x$ is the terminal missing label for some maximal $x_{i}$-path $C_{i, j^{\prime}}$.

Proof Suppose that the number of vertices of $\mathcal{G}$ is $m$. Let $k$ be the number of existing directed edges of $\mathcal{G}$ with label $x$. Then $m-k$ is equal to the number of initial missing edges of $\widehat{\mathcal{G}}$ with label $x$ and is also equal to the number of terminal missing edges of $\widehat{\mathcal{G}}$ with label $x$. The lemma follows.

We shall let:

$$
L_{*}=\left\{a_{1}, b_{1}, \ldots, a_{g}, b_{g}\right\}
$$

The proof of the following lemma is obvious.

Lemma 9.5 Let $\mathcal{G}$ be a finite connected $L$-labeled directed graph such that $\mathcal{G}$ is folded and such that $\widehat{\mathcal{G}}$ contains no $x_{i}$-loops for any $i=1, \ldots, b$. If $x \in L_{*} \cup L_{*}^{-1}$ is the initial or terminal missing label of some $C_{b, j}$ at a vertex $v$, then $x^{-1}$ must also be the terminal or initial missing label of some $C_{b, j^{\prime}}$ respectively at the same vertex $v$.

By Lemma 9.3, it is enough to show that the graph $\mathcal{G}_{0}$ embeds in a finite $L$-regular graph $\mathcal{G}_{*}$ such that $\widehat{\mathcal{G}}_{*}$ contains no $x_{i}$-loop (based at any vertex) representing the word $x_{i}^{k}$ for any $i=1, \ldots, b$ and $k=1, \ldots, m_{*}-1$, where $m_{*}$ is the number of vertices of $\mathcal{G}_{*}$. Indeed, assuming such $\mathcal{G}_{*}$ is found, we have:

(1) $G=L\left(\mathcal{G}_{*}, v_{0}\right)$ is an index $m_{*}$ subgroup of $F$ (Lemma 9.3);

(2) $G$ contains $H$ as a subgroup but does not contain any of the elements $y_{1}, \ldots, y_{a}$ (because $\mathcal{G}_{0}$ is an embedded subgraph of $\mathcal{G}_{*}$ and $y_{1}, \ldots, y_{a}$ are represented by nonclosed paths with initial vertex $v_{0}$ );

(3) $G$ does not contain any of the elements $x_{i}^{k}, i=1, \ldots, b, k=1, \ldots, m_{*}-1$ (because $\widehat{\mathcal{G}}_{*}$ has no $x_{i}$-loop representing the word $x_{i}^{k}$ for any $i=1, \ldots, b$ and any $\left.k=1, \ldots, m_{*}-1\right)$.

In the rest of this section we show that such a graph $\mathcal{G}_{*}$ exists.

Definition Let $\mathcal{G}$ be a finite, connected, $L$-labeled, directed graph such that $\mathcal{G}$ is folded and such that $\widehat{\mathcal{G}}$ contains no $x_{i}$-loops for any $i=1, \ldots, b$. A graph $\mathcal{G}^{\prime}$ is called a good extension of $\mathcal{G}$ if:

(1) $\mathcal{G}^{\prime}$ is a finite, connected, $L$-labeled, directed graph;

(2) $\mathcal{G}^{\prime}$ contains $\mathcal{G}$ as an embedded subgraph;

(3) $\mathcal{G}^{\prime}$ is folded; 
(4) $\widehat{\mathcal{G}}^{\prime}$ contains no $x_{i}$-loops for all $i=1, \ldots, b$.

Definition Let $\mathcal{G}$ be a finite, connected, $L$-labeled, directed graph such that $\mathcal{G}$ is folded and such that $\widehat{\mathcal{G}}$ contains no $x_{i}$-loops for any $i=1, \ldots, b$. A graph $\mathcal{G}^{\prime}$ is called a perfect extension of $\mathcal{G}$ if:

(1) $\mathcal{G}^{\prime}$ is a finite, connected, $L$-labeled, directed graph;

(2) $\mathcal{G}^{\prime}$ contains $\mathcal{G}$ as an embedded subgraph;

(3) $\mathcal{G}^{\prime}$ is $L$-regular;

(4) $\widehat{\mathcal{G}}^{\prime}$ contains no loop representing the word $x_{i}^{k}$ for any $i=1, \ldots, b$ and any $k=1, \ldots, m-1$, where $m$ is the number of vertices of $\mathcal{G}^{\prime}$.

We shall describe a canonical procedure for constructing a finite sequence of graphs $\mathcal{G}_{0}, \mathcal{G}_{1}, \ldots, \mathcal{G}_{n}$ such that:

(1) $\mathcal{G}_{0}$ is the graph given above;

(2) $\mathcal{G}_{p+1}$ is a good extension of $\mathcal{G}_{p}$ for each $p=1, \ldots, n-2$ (if $n>1$ );

(3) $\mathcal{G}_{n}$ is a perfect extension of $\mathcal{G}_{n-1}$.

Obviously if such a sequence of graphs can be constructed, then $\mathcal{G}_{n}$ will be the graph which we seek.

We divide our discussion into three cases: $b=1, b=2$ and $b>2$. We need the following definitions for all the three cases.

Definitions For $g>0$, let $\mathcal{G}$ be a finite, connected, $L$-labeled, directed graph such that $\mathcal{G}$ is folded and such that $\widehat{\mathcal{G}}$ contains no $x_{i}$-loops for any $i=1, \ldots, b$. Let $x \in L_{*} \cup L_{*}^{-1}$.

A maximal $x_{b}$-path $C_{b, j}$ of $\widehat{\mathcal{G}}$ is called a type $I$ maximal $x_{b}$-path with missing label $x$ if:

(1) $x$ is the initial missing label of $C_{b, j}$ and $x^{-1}$ is the terminal missing label of the same path $C_{b, j}$;

(2) the initial vertex and the terminal vertex of $C_{b, j}$ are the same vertex.

A maximal $x_{b}$-path $C_{b, j}$ of $\widehat{\mathcal{G}}$ is called a type $I I$ maximal $x_{b}$-path with missing label $x$ (again we assume that $g>0$ ) if $x$ is the initial missing label of $C_{b, j}$ and is also the terminal missing label of the same path. 
Figure 5 (a) illustrates a pair of type I maximal $x_{b}$-paths, and Figure 5 (b) shows a pair of type II maximal $x_{b}$-paths. In these figures, a missing label is represented by a dotted, labeled edge; the initial missing label is given at the left end of a path and the terminal one at the right end.

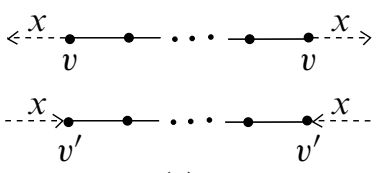

(a)

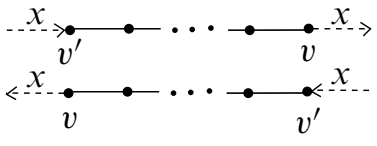

(b)

Figure 5

Case $1 \quad b=1$

In this case, we have $g>0$, free basis $L=L_{*}=\left\{a_{1}, b_{1}, \ldots, a_{g}, b_{g}\right\}$, and a surface $S^{-}$with a single boundary component, which is freely isotopic to a loop representing the commutator $x_{b}=x_{1}=\left[a_{1}, b_{1}\right] \cdots\left[a_{g}, b_{g}\right]$.

Start with the graph $\mathcal{G}_{0}$. Since $\widehat{\mathcal{G}}_{0}$ has no $x_{b}$-loops, every maximal $x_{b}$-path in $\widehat{\mathcal{G}}_{0}$ has both initial and terminal missing labels. Suppose that $x$ is a missing label. Then $x \in L_{*} \cup L_{*}^{-1}$. By Lemmas 9.4 and 9.5, we have maximal $x_{b}$-paths (possibly nondistinct) $C_{b, j}, C_{b, j^{\prime}}, C_{b, k}, C_{b, k^{\prime}}$, with missing labels as illustrated on the left hand side in Figure 6. Note that although we draw these paths separately, they may actually share some common vertices. Also a path we draw may not be simply connected, ie some of its vertices maybe the same vertex. Note also that instead of drawing an oriented edge with label $x^{-1}$, we often draw, equivalently, an edge with the opposite orientation and with label $x$ (ie every edge we draw shall be considered as two directed edges with opposite orientations and inverse labels). Also, a pair of paths in the figure may in fact be non-distinct (eg maybe $C_{b, j}=C_{b, j^{\prime}}$ ). However, the four paths given on the left hand side in Figure 6 must satisfy $C_{b, j} \neq C_{b, k}$ and $C_{b, j^{\prime}} \neq C_{b, k^{\prime}}$.

Operation 1 Suppose that the four maximal $x_{b}$-paths given in Figure 6 satisfy: $C_{b, j} \neq C_{b, j^{\prime}}, C_{b, k} \neq C_{b, k^{\prime}}$, and either $C_{b, j} \neq C_{b, k^{\prime}}$ or $C_{b, j^{\prime}} \neq C_{b, k}$. Then we may perform the following operation: add a new vertex $w$; for each letter in $L-\{x\}$ add a new edge with both its initial and terminal vertices at $w$ and with that letter as the label; add a new edge with label $x$, initial vertex $v$ and terminal vertex $w$; and add a new edge with label $x$, initial vertex $w$ and terminal vertex $v^{\prime}$. A piece of the resulting graph is shown on the right hand side in Figure 6, where there are exactly $2 g-1$ single-edge loops at the new vertex $w$, with labels one each from $L-\{x\}$.

We claim that the resulting graph is a good extension of $\mathcal{G}_{0}$, and that the number of maximal $x_{b}$-paths in the new graph is reduced. Indeed, the new graph is obviously 

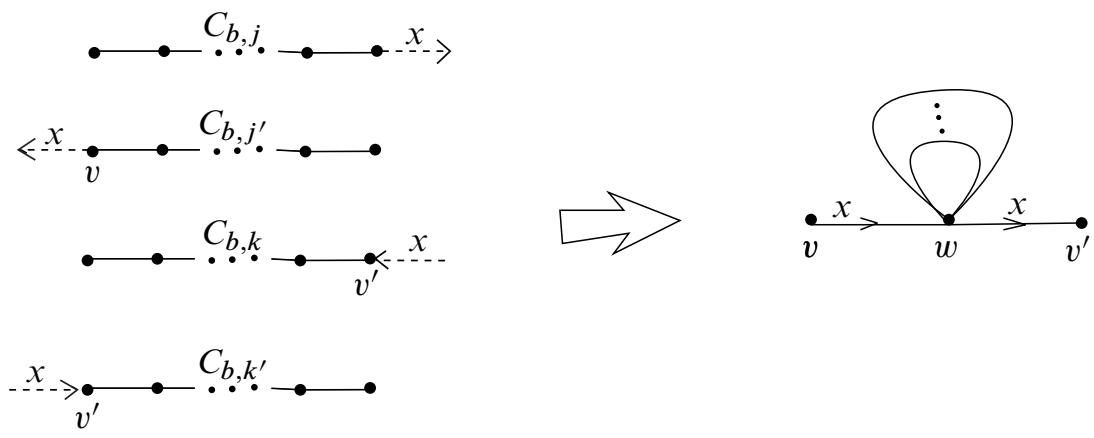

Figure 6

finite, connected, $L$-labeled and contains $\mathcal{G}_{0}$ as an embedded subgraph, and one can check that it is folded. One can also check that the number of maximal $x_{b}$-paths in the new graph is reduced. Namely the maximal $x_{b}$-paths $C_{b, j}$ and $C_{b, j^{\prime}}$ are joined into a single maximal $x_{b}$-path, as are $C_{b, k}$ and $C_{b, k^{\prime}}$. No extra maximal $x_{b}$-paths are created, since all the added edges are used in the two new maximal $x_{b}$-paths. In particular no $x_{b}$-loops are created.

Operation 2 Suppose that the four maximal $x_{b}$-paths given in Figure 6 satisfy: $C_{b, j} \neq C_{b, k^{\prime}}, C_{b, j^{\prime}} \neq C_{b, k}$ and either $C_{b, j} \neq C_{b, j^{\prime}}$ or $C_{b, k} \neq C_{b, k^{\prime}}$. Then we may perform the following operation: add a new edge $e$ with the label $x$, initial vertex $v$ and terminal vertex $v^{\prime}$. Again one can easily check that the resulting graph from an operation 2 is a good extension of the old graph, and the number of maximal $x_{b}$-paths in the new graph is reduced.

For each pair $\left\{x, x^{-1}\right\}$, we perform Operations 1 and 2 as many times as possible. Since each operations reduces the number of maximal $x_{b}$-paths, this process will terminate in a graph $\mathcal{G}_{1}$ for which neither Operation 1 nor Operation 2 may be applied for any pair $\left\{x, x^{-1}\right\}$.

\section{Lemma 9.6}

(1) $\mathcal{G}_{1}$ is a good extension of $\mathcal{G}_{0}$.

(2) In $\widehat{\mathcal{G}}_{1}$, for each letter pair $\left\{x, x^{-1}\right\}$, one of the following holds:

I every maximal $x_{b}$-path with $x$ or $x^{-1}$ as an initial or terminal missing label is of Type I; or

II there are only two maximal $x_{b}$-paths which have $x$ or $x^{-1}$ as a missing label; moreover one of the two paths is a type II maximal $x_{b}$-path with $x$ as its missing label and the other path is a type II maximal $x_{b}$-path with $x^{-1}$ as a missing label; or 
III there is no maximal $x_{b}$-path with $x$ or $x^{-1}$ as an initial or terminal missing label.

Proof Part (1) of the lemma holds since we have checked that Operations 1 and 2 always yield good extensions.

To show part (2) of the lemma, fix a letter pair $\left\{x, x^{-1}\right\}$, and suppose that case III does not happen. Then as discussed above, in $\widehat{\mathcal{G}}_{1}$ we have maximal $x_{b}$-paths $C_{b, j}, C_{b, j^{\prime}}$, $C_{b, k}$ and $C_{b, k^{\prime}}$ (possibly non-distinct) as shown in Figure 6. If $C_{b, j}=C_{b, j^{\prime}}$, then we must have $C_{b, k}=C_{b, k^{\prime}}$, and vice versa. For otherwise Operation 2 would apply. It follows that if $C_{b, j}=C_{b, j^{\prime}}$ or $C_{b, k}=C_{b, k^{\prime}}$ are the same path, then any other such pair of paths must be the same, ie Case I holds. So we may assume that for any two pairs of maximal $x_{b}$-paths $\left(C_{b, j}, C_{b, j^{\prime}}\right)$ and $\left(C_{b, k}, C_{b, k^{\prime}}\right)$ as shown in Figure 6, we have $C_{b, j} \neq C_{b, j^{\prime}}$ and $C_{b, k} \neq C_{b, k^{\prime}}$. But since Operation 1 does not apply to them, we must have $C_{b, j}=C_{b, k^{\prime}}$ and $C_{b, j^{\prime}}=C_{b, k}$. Thus we have a pair of Type II maximal $x_{b}$-paths with $x$ and $x^{-1}$ as their missing labels, respectively. Again since Operation 1 does not apply, there is at most one such pair, ie case II occurs.

Operation 3 Suppose that $\widehat{\mathcal{G}}_{1}$ satisfies Property I of Lemma 9.6, for some letter pair $\left\{x, x^{-1}\right\}$. Let $k$ be the total number of pairs of Type I maximal $x_{b}$-paths with missing labels in $\left\{x, x^{-1}\right\}$. Then we may perform the operation shown in Figure 7 to change all of them into a single pair of Type II maximal $x_{b}$-paths with a letter different from $x$ or $x^{-1}$ as the missing label. For illustration, in Figure 7 we assume that $k=3$ and $x=a_{1}$. We add three new vertices $w_{1}, w_{2}, w_{3}$, two new edges with label $b_{1}$, and new loops at each $w_{i}$ having labels one each from $L_{*}-\left\{a_{1}, b_{1}\right\}$. The three pairs of type I maximal $x_{b}$-paths with missing label $a_{1}$ and $a_{1}^{-1}$ become one pair of type II maximal $x_{b}$-paths with missing label $b_{1}$ and $b_{1}^{-1}$. All the added new edges are used in this pair of new maximal $x_{b}$-paths. If the missing labels for the type I pairs are $b_{1}$ and $b_{1}^{-1}$ instead of $a_{1}$ and $a_{1}^{-1}$, then in Figure 7 we exchange the letters $a_{1}$ and $b_{1}$. In general, for arbitrary $k$ and arbitrary missing label pair $\left\{x, x^{-1}\right\}$, it should be clear how to make a similar operation.

Again one can check that, if Operation 3 is applied to a good extension, then the resulting graph is a good extension. Also, after Operation 3 is applied, the $k$ pairs of Type I paths are replaced with a single pair of Type II paths, whose missing labels are different from $\left\{x, x^{-1}\right\}$. If $\left\{x, x^{-1}\right\}=\left\{a_{i}, a_{i}^{-1}\right\}$, then the new label pair is $\left\{b_{i}, b_{i}^{-1}\right\}$, and vice versa. Note that after applying Operation 3 , the total number of maximal $x_{b}$-paths cannot increase, although it may stay the same.

Now, starting with the graph $\mathcal{G}_{1}$, we perform Operation 3 as many times as possible. We are left with a graph, $\mathcal{G}_{2}$, whose maximal $x_{b}$-paths are all of Type II. Suppose $\mathcal{G}_{2}$ has more than one pair of Type II maximal $x_{b}$-paths with missing labels in $\left\{x, x^{-1}\right\}$. 


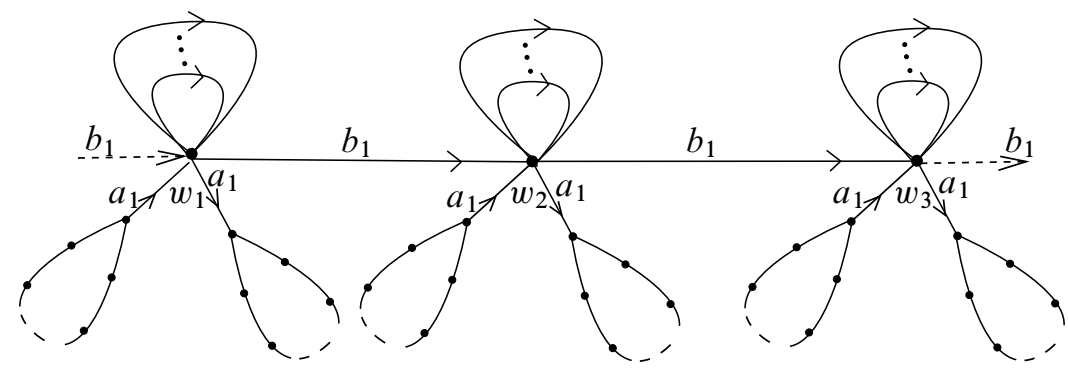

Figure 7

Then we may apply Operation 2. The effect is to replace a pair of Type II paths with a single Type II path. Therefore, after performing Operation 2 repeatedly, we arrive at a graph $\mathcal{G}_{3}$, such that, for every missing label pair $\left\{x, x^{-1}\right\}$, there is exactly one pair of Type II paths corresponding to that pair, and there are no other maximal $x_{b}$-paths with $x$ or $x^{-1}$ as missing label. Note that $\mathcal{G}_{3}$ cannot be $L$-regular, since our operations thus far never create an $x_{b}$-loop.

Operation 4 We add a single vertex $w$, and then add the appropriate edges to make the graph folded, with no missing labels from $L_{*}$. This is illustrated in Figure 8 in the case where there are exactly three pairs of maximal $x_{b}$-paths, with missing labels $\left\{x, x^{-1}\right\},\left\{x^{\prime}, x^{\prime-1}\right\},\left\{x^{\prime \prime}, x^{\prime \prime}-1\right\}$. The loops at the added vertex $w$ have labels from the remaining labels in $L_{*}$.

The resulting graph $\mathcal{G}_{4}$ is what we wanted, ie it is a perfect extension of $\mathcal{G}_{3}$. Indeed, it is easy to check that the graph is finite, connected, and folded, with $\mathcal{G}_{3}$ as an embedded subgraph; also, in the current case, $L_{*}=L$, so $\mathcal{G}_{4}$ is $L$-regular. So we only need to check that $\widehat{\mathcal{G}}_{4}$ has no $x_{b}$-loops representing the word $x_{b}^{k}$ for any $k=1, \ldots, m-1$, where $m$ is the number of vertices of $\mathcal{G}_{4}$. To see this holds, refer to Figure 8, and trace out an $x_{b}$-loop, starting at any vertex of $\mathcal{G}_{4}$. One sees that an $x_{b}$-loop does not occur until every edge of $\widehat{\mathcal{G}}_{4}$ has been used exactly once (in particular each maximal $x_{b}$-path $C_{b, j}$ in $\widehat{\mathcal{G}}_{3}$ has been traced out exactly once). This $x_{b}$-loop represents the word $x_{b}^{m}$, since $\mathcal{G}_{4}$ is $L$-regular and contains $m$ vertices. This completes the proof of Theorem 9.1 when $b=1$.

Case $2 b=2$

In this case, the free basis $L=\left\{a_{1}, b_{1}, \ldots, a_{g}, b_{g}, x_{1}\right\}$, and we also have $x_{b}=x_{2}=$ $\left[a_{1}, b_{1}\right] \cdots\left[a_{g}, b_{g}\right] x_{1}$ (note that $g>0$ in this case too). Recall $L_{*}=\left\{a_{1}, b_{1}, \ldots, a_{g}, b_{g}\right\}$. 

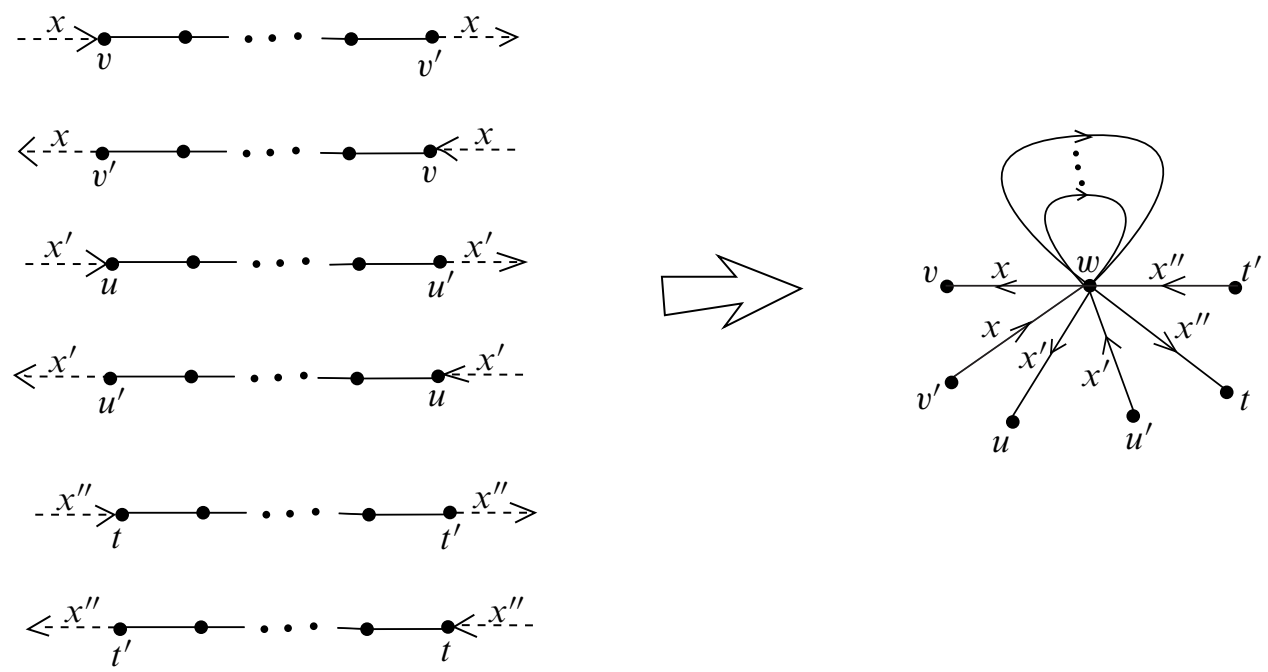

Figure 8

The first step in this case is to construct a good extension graph $\mathcal{G}_{1}$ of $\mathcal{G}_{0}$ such that $\widehat{\mathcal{G}}_{1}$ has no missing label pairs from $L_{*} \cup L_{*}^{-1}$. The procedure for constructing such $\mathcal{G}_{1}$ is the same as that given in Case 1. Only in the current case, after applying an Operation 1 , or 3 , or 4 , we may increase the number of maximal $x_{b}$-paths with missing label $x_{1}$ and may also increase the number of maximal $x_{1}$-paths. But no $x_{1}$-loops will be created because during each of these operations no new edge with label $x_{1}$ is added.

Let $\mathcal{G}_{1}$ be the resulting good extension graph after all missing edges with labels from $L_{*}$ are eliminated. So every maximal $x_{b}$-path $C_{b, j}$ in $\widehat{\mathcal{G}}_{1}$ has both its initial and terminal missing labels being $x_{1}$. In the current case, we also need to consider maximal $x_{1}$-paths. Their missing labels are of course always $x_{1}$. Note also that in $\widehat{\mathcal{G}}_{1}$ the number of maximal $x_{b}$-paths is the same as the number of maximal $x_{1}$-paths (since $b=2$ and since $x_{1}$ is now the only missing label from $L$ ). Suppose that there are at least three maximal $x_{b}$-paths in $\widehat{\mathcal{G}}_{1}$. We illustrate three such paths in Figure 9 left hand side. Thus there are at least three maximal $x_{1}$-paths, which are shown in Figure 9 right hand side. Since the graph $\mathcal{G}_{1}$ is connected and folded, the vertices shown in Figure 9 satisfy $v_{1} \neq v_{3} \neq v_{5} \neq v_{1}, v_{2} \neq v_{4} \neq v_{6} \neq v_{2}$. Also $C_{1,1}, C_{1,2}, C_{1,3}$ are mutually disjoint embedded paths. By adding to $v_{2}$ a subgraph which is shown in Figure 10 (in the figure, the loops have labels one each from $L_{*}$ ), we may assume that $v_{2} \neq v_{3}$ and $v_{2} \neq v_{5}$. We may also assume that $v_{2}=w_{2}$, and $v_{3} \neq w_{1}$. Now we simply add an edge with label $x_{1}$ to $\mathcal{G}_{1}$ pointing from $v_{2}$ to $v_{3}$. Then the new graph is folded with one less number of maximal $x_{b}$-paths, and with no $x_{i}$-loops created, $i=1,2$ (since $v_{3}$ is not an end vertex in the path $C_{1,1}$ ). 

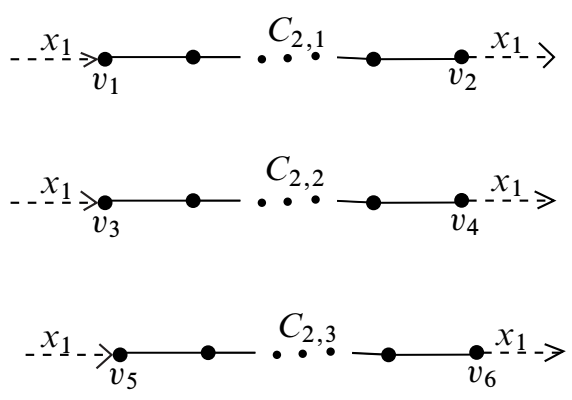
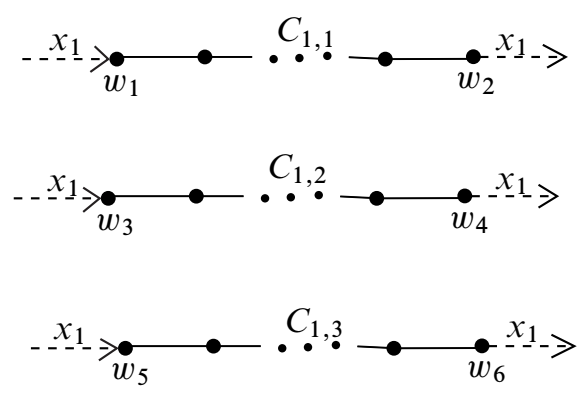

Figure 9

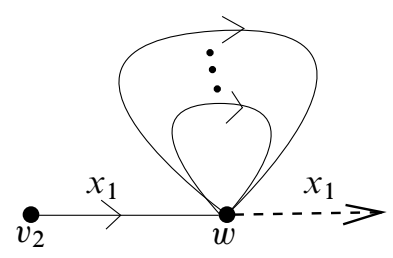

Figure 10

So we may assume we have a graph, still denoted $\mathcal{G}_{1}$, such that $\widehat{\mathcal{G}}_{1}$ has at most two maximal $x_{b}$-paths. If there is only one such path, we simply add a single edge with the missing label $x_{1}$. The resulting graph is what we requested. So we may assume that there are exactly two such paths, as shown in Figure 11. Again we have $v_{1} \neq v_{3}$, $v_{2} \neq v_{4}$ and may assume $v_{2} \neq v_{3}$ and $v_{2}=w_{2}$. If $v_{3} \neq w_{1}$, then we simply add an edge with label $x_{1}$ pointing from $v_{2}$ to $v_{3}$. So we may assume that $v_{3}=w_{1}$. In such case we cannot add an edge from $v_{2}$ to $v_{3}$ with label $x_{1}$ since that will create a graph which has an $x_{1}$-loop but is not yet $L$-regular. The initial and terminal vertices of these paths are illustrated in the first two rows of Figure 12. Now we take an identical copy $\mathcal{G}_{1}^{\prime}$ of the graph $\mathcal{G}_{1}$. The corresponding maximal $x_{i}$-paths in $\widehat{\mathcal{G}}_{1}^{\prime}$ are denoted by $C_{i, j}^{\prime}$. The union of these paths from the two graphs are shown in Figure 12. We now connect these two graphs together as follows: add an edge from $v_{2}$ to $v_{1}^{\prime}$, add an edge from $v_{4}$ to $v_{3}^{\prime}$, add an edge from $v_{2}^{\prime}$ to $v_{3}$, and add an edge from $v_{4}^{\prime}$ to $v_{1}$, all with label $x_{1}$. Then one can easily check that the resulting graph is what we requested. This proves the theorem when $b=2$.

Case $3 \quad b \geq 3$

Again we first eliminate all missing labels belonging to $L_{*}=\left\{a_{1}, b_{1}, \ldots, a_{g}, b_{g}\right\}$, with a similar method as in Case 2. Namely during the relevant operations, no new edges with label $x_{i}, i=1, \ldots, b-1$ are added. Note that the resulting graph $\mathcal{G}_{1}$ must 

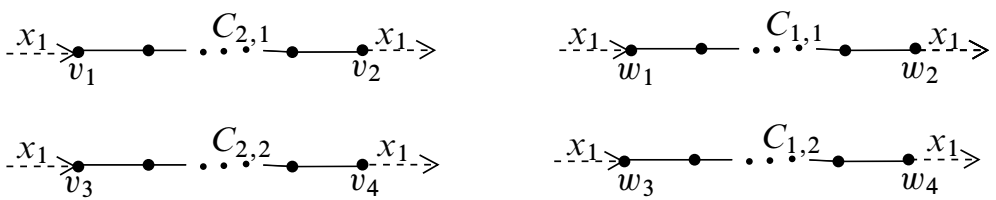

Figure 11
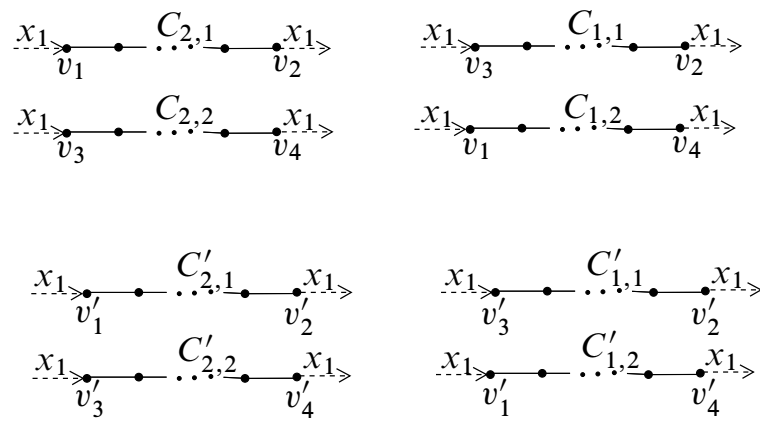

Figure 12

have missing $x_{i}$-labels for each $i=1, \ldots, b-1$ (since no $x_{i}$-loops were created). Let $C_{b, j}$ be a maximal $x_{b}$-path in $\widehat{\mathcal{G}}_{1}$. Its initial missing label might not be the same as its terminal missing label. If so, we call such path a maximal $x_{b}$-path with mixed missing labels. Note that for each $i=1, \ldots, b-1$, the number of initial missing $x_{i}$ labels is equal to the number of terminal missing $x_{i}$ labels. It follows that if the graph $\widehat{\mathcal{G}}_{1}$ has a maximal $x_{b}$-path $C_{b, j}$ with mixed missing labels and with $x_{1}$, say, as the terminal missing label, then there must be another maximal $x_{b}$-path $C_{b, j^{\prime}}$ with mixed missing labels and with $x_{1}$ as the initial missing label, and vice versa.

Take $b-1$ maximal $x_{b}$-paths with mutually distinct terminal missing labels. Such $b-1$ paths must exist as we already noted. By adding a subgraph as shown in Figure 13 to the $b-1$ terminal vertices of the maximal $x_{b}$-paths (in Figure 13, loops at the vertex $w$ have labels from $L_{*}$ ) we may assume that the terminal vertex is different from the initial vertex for every one of these $b-1$ maximal $x_{b}$-paths, and we may also assume that the graph has a maximal $x_{b}$-path with mixed missing labels. Note that the operation given in Figure 13 results in a good extension, and does not change the number of maximal $x_{b}$-paths. Together with the notes given in the preceding paragraph, we see that for any given label $x_{i}, i=1, \ldots, b-1$, we may change our graph with the operation given in Figure 13 so that the resulting graph has a maximal $x_{b}$-path with mixed missing labels and with $x_{i}$ as the terminal missing label, without increasing the total number of maximal $x_{b}$-paths. 


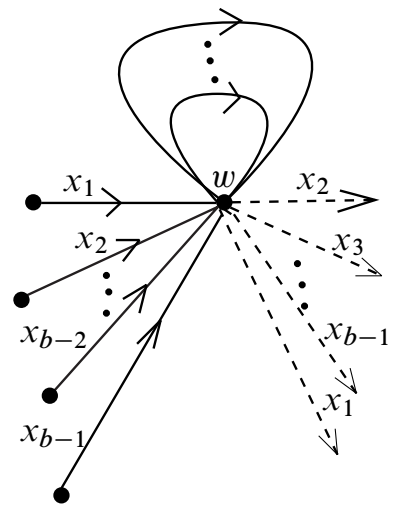

Figure 13

Now suppose that there is a maximal $x_{b}$-path $C_{b, 1}$ with mixed missing labels and with $x_{1}$, say, as terminal missing label, and suppose that there are at least two maximal $x_{1}$-paths in the graph. Then there are at least two maximal $x_{b}$-paths $C_{b, 2}$ and $C_{b, 3}$ which have $x_{1}$ as their initial missing label. The situation is illustrated in Figure 14. We may assume that $v_{2}=w_{2}$. We may assume one of $v_{3}$ and $v_{5}$, say $v_{3}$, is different from $w_{1}$ since the graph is folded. Now we add an edge connecting $v_{2}$ to $v_{3}$ with the label $x_{1}$. Then no $x_{b}$-loop is created since $C_{b, 1}$ has mixed missing labels. Also no $x_{1}$-loop is created since $v_{2} \neq v_{3}$ and $v_{3} \neq w_{1}$. But the number of maximal $x_{b}$-paths is reduced.
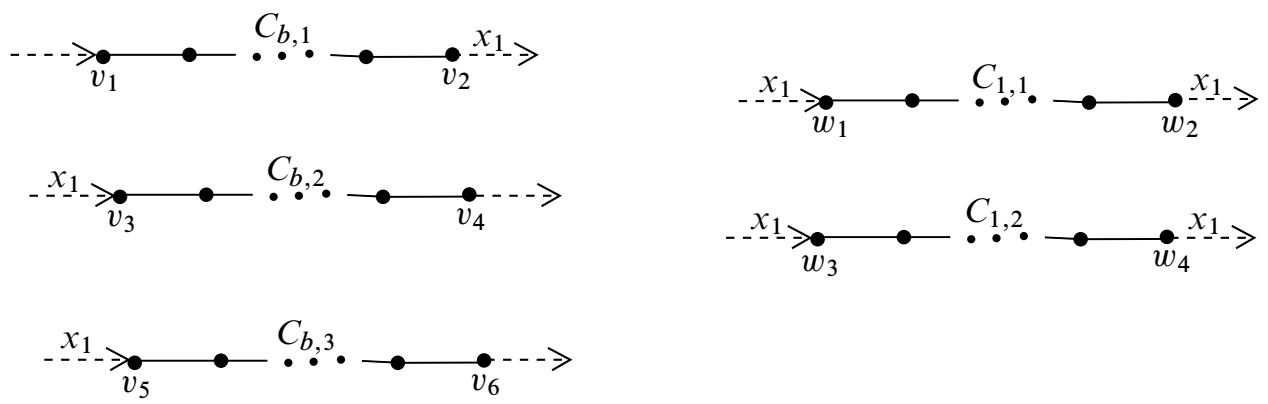

Figure 14

Repeating such operation, we may assume that our graph has exactly one maximal $x_{i}$-path for each $i=1,2, \ldots, b-1$. Thus there are exactly $b-1$ maximal $x_{b}$-paths, $C_{b, i}, i=1, \ldots, b-1$. We may assume that the initial missing label of $C_{b, i}$ is $x_{i}$ for $i=1, \ldots, b-1$. Then the terminal missing label of $C_{b, i}$ is $x_{\sigma(i)}$ for some permutation $\sigma$ of the set $\{1,2, \ldots, b-1\}$. Suppose that $\sigma$ has order $n$. We take $n$ copies of the graph and connect them as indicated in Figure 15. 


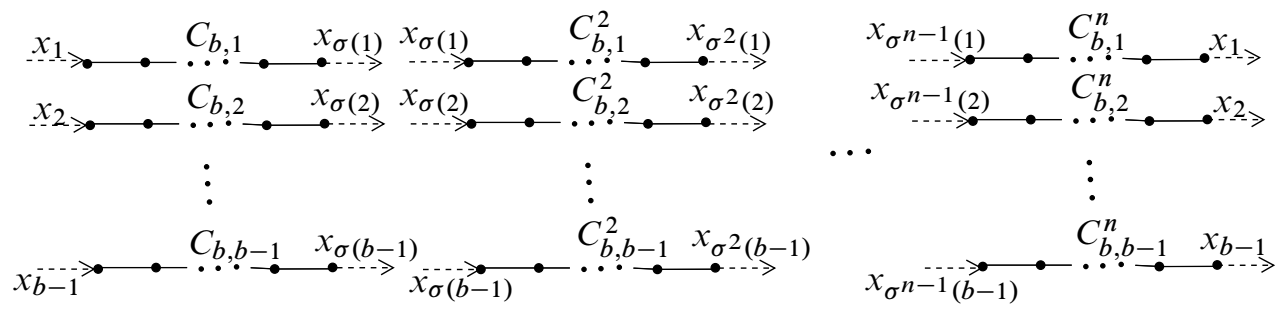

Figure 15

The resulting graph again has exactly $b-1$ maximal $x_{b}$-paths, but the permutation of their missing labels is the identity now. By adding the subgraph in Figure 13 to the graph at the right side, the permutation becomes a cyclic permutation $i \rightarrow i+1$, $i=1, \ldots, b-1$ (defined mod $b-1$ ). Now we simply fill in $b-1$ edges at the obvious places with the right labels. The resulting graph is what we wanted.

The proof of Theorem 9.1 is finally completed.

Remark 9.7 Note that the arguments in this section actually show that if $\mathcal{G}_{\text {\# }}$ is a finite, $L$-labeled, directed, folded graph with base vertex $v_{0}$, with corresponding subgroup $\left.G_{\#}=L\left(\mathcal{G}_{\#}, v_{0}\right) \subset F=\pi_{1}\left(S^{-}, s\right)\right)$, such that

- $\mathcal{G}_{\#}$ does not contain any loop representing the word $x_{i}^{j}$ for any $i=1, \ldots, b$, $j \in \mathbb{Z}-\{0\}$, and

- $y_{1}, \ldots, y_{r}$ are some fixed, non-closed paths based at $v_{0}$ in $\mathcal{G}_{\#}$, then there is a finite, connected, $L$-regular graph $\mathcal{G}_{*}$ such that

- $\mathcal{G}_{*}$ contains $\mathcal{G}_{\#}$ as an embedded subgraph, and thus in particular $y_{1}, \ldots, y_{a}$ remain non-closed paths based at $v_{0}$ in $\mathcal{G}_{*}$, and

- $\mathcal{G}_{*}$ contains no loops representing the word $x_{i}^{j}$, for each $i=1, \ldots, b, j=$ $1, \ldots, m_{*}-1$, where $m_{*}$ is the number of vertices of $\mathcal{G}_{*}$.

That is, the graph $\mathcal{G}_{*}$ is a perfect extension of $\mathcal{G}_{\#}$.

In terms of groups, $L\left(\mathcal{G}_{*}, v_{0}\right)$ represents a subgroup $G_{*}$ of $F$ of index $m_{*}$ such that

- $G_{*}$ contains $G_{\#}$ as a subgroup;

- $G_{*}$ does not contain any of the elements $x_{i}^{j}, i=1, \ldots, b, j=1, \ldots, m_{*}-1$;

- $G_{*}$ does not contain any of the elements $y_{1}, \ldots, y_{r}$ (considered as words in the generators in $L \cup L^{-1}$ ).

This remark will be used later in Section 11. 


\section{Lifting immersions to embeddings}

We recall some of the notations from earlier sections. Each of $i, i_{*}$ denotes a number 1 or 2 such that $\left\{i, i_{*}\right\}=\{1,2\}$. We have the universal covering maps $p: \mathbb{H}^{3} \rightarrow$ $M, p \mid: \mathbb{H}^{3} \backslash \mathcal{B} \rightarrow M^{-}, p_{i}: X_{i}=\widetilde{S}_{i} \times I \rightarrow Y_{i}=S_{i} \times I$, and $p_{i} \mid: X_{i}^{-}=\widetilde{S}_{i}^{-} \times$ $I \rightarrow Y_{i}^{-}=S_{i}^{-} \times I$. We have a local isometry $f_{i}: Y_{i}=S_{i} \times I \rightarrow M$ such that $f_{i} \mid:\left(Y_{i}^{-}=S_{i}^{-} \times I, \partial_{p} Y_{i}^{-}=\partial S_{i}^{-} \times I\right) \rightarrow(M, \partial M)$ is a proper map and such that $f_{i}: \partial S_{i}^{-} \rightarrow \partial M$ is an embedding. The surface $S_{i}^{-}$has $n_{i}$ boundary components $\left\{\beta_{i, j}, j=1, \ldots, n_{i}\right\}$ with induced orientation. There are $d=\Delta n_{1} n_{2}$ intersection points $\left\{t_{j}, j=1, \ldots, d\right\}$ between $f_{1}\left(\partial S_{1}^{-}\right)$and $f_{2}\left(\partial S_{2}^{-}\right)$in $\partial M$. The points in $f_{i}^{-1}\left\{t_{1}, \ldots, t_{d}\right\}$ are $\left\{t_{i, j, k}, j=1, \ldots, n_{i}, k=1, \ldots, d_{i}\right\}$, where $d_{i}=\Delta n_{i_{*}}$, indexed so that $\left\{t_{i, j, k}, k=1, \ldots, d_{i}\right\}$ are contained successively in the component $\beta_{i, j}$ (following the orientation of $\beta_{i, j}$ ) for each $j=1, \ldots, n_{i}$. We constructed a metrically complete, convex, hyperbolic 3-manifold $J_{i}$ with a local isometry $g_{i}: J_{i} \rightarrow Y_{i}$ such that $g_{i} \mid:\left(J_{i}^{-}, \partial_{p} J_{i}^{-}\right) \rightarrow\left(Y_{i}^{-}, \partial_{p} Y_{i}^{-}\right)$is a proper map. The parabolic boundary of $J_{i}^{-}, \partial_{p} J_{i}^{-}$, has exactly $d$ components $\left\{D_{i, j, k}, j=1, \ldots, n_{i}, k=1, \ldots, d_{i}\right\}$, and the topological center point of $D_{i, j, k}$ is denoted $b_{i, j, k}$. We have $g_{i}\left(b_{i, j, k}\right)=t_{i, j, k}$. For each sufficiently large integer $n>0$, we constructed a compact, convex, hyperbolic 3-manifold $C_{n}\left(J_{i}^{-}\right)$which contains $J_{i}^{-}$as an embedded submanifold, and a local isometry $g_{i}: C_{n}\left(J_{i}^{-}\right) \rightarrow Y_{i}$, extending the map $g_{i}: J_{i}^{-} \rightarrow Y_{i}$.

Base point convention From now on, we will fix $t_{1}$ as a basepoint for each of $M$, $M^{-}$and $\partial M$, and $t_{i, 1,1}$ will be the base point for each of $S_{i}, S_{i}^{-}, Y_{i}$ and $Y_{i}^{-}$. After re-ordering $\left\{t_{1}, \ldots, t_{d}\right\}$, we may assume that $f_{i}\left(t_{i, 1,1}\right)=t_{1}$ for $i=1,2$, and that the point $\tilde{b}=\widetilde{S}_{1} \cap \widetilde{S}_{2} \cap \partial B_{\infty}$ is in $p^{-1}\left(t_{1}\right)$. The point $\widetilde{b}$ will be the base point for each of $\mathbb{H}^{3}, X_{i}, X_{i}^{-}, \widetilde{S}_{i}, \widetilde{S}_{i}^{-}$, and $\partial B_{\infty}$. The point $b_{i, 1,1}$ will be the base point for each of $J_{i}$, $J_{i}^{-}, \widehat{J}_{i}$ and $C_{n}\left(J_{i}^{-}\right)$. Under these choices of base points, we can identify, as in Section 4, each of $\pi_{1}\left(M, t_{1}\right)$ and $\pi_{1}\left(M^{-}, t_{1}\right)$ with the group $\Gamma$; identify each of $\pi_{1}\left(S_{i}, t_{i, 1,1}\right)$, $\pi_{1}\left(S_{i}^{-}, t_{i, 1,1}\right), \pi_{1}\left(Y_{i}, t_{i, 1,1}\right), \pi_{1}\left(Y_{i}^{-}, t_{i, 1,1}\right)$ with the quasi-Fuchsian group $\Gamma_{i} \subset \Gamma$; and identify $\pi_{1}\left(\partial M, t_{1}\right)$ with the stabilizer of $\infty$ in $\Gamma$. Under such identifications, the induced map $f_{i}^{*}: \pi_{1}\left(S_{i}, t_{i, 1,1}\right)=\Gamma_{i} \rightarrow \pi_{1}\left(M, t_{1}\right)=\Gamma$ is the inclusion homomorphism, and each of the inclusion maps $\left(S_{i}, t_{i, 1,1}\right) \subset\left(Y_{i}, t_{i, 1,1}\right),\left(S_{i}^{-}, t_{i, 1,1}\right) \subset\left(Y_{i}^{-}, t_{i, 1,1}\right)$, $\left(S_{i}^{-}, t_{i, 1,1}\right) \subset\left(S_{i}, t_{i, 1,1}\right),\left(Y_{i}^{-}, t_{i, 1,1}\right) \subset\left(Y_{i}, t_{i, 1,1}\right)$ and $\left(M^{-}, t_{1}\right) \subset\left(M, t_{1}\right)$ induces the identity isomorphism on the fundamental groups.

Choice of a free basis for $\Gamma_{i}$ Recall that $n_{i}$ is the number of boundary components of the truncated surface $S_{i}^{-}$. Let $g_{i}$ be the genus of $S_{i}^{-}$. As in Section 9, the group $\Gamma_{i}=\pi_{1}\left(S_{i}^{-}, t_{i, 1,1}\right)=\pi_{1}\left(Y_{i}, t_{i, 1,1}\right)=\pi_{1}\left(S_{i}, t_{i, 1,1}\right)=\pi_{1}\left(Y_{i}, t_{i, 1,1}\right)$ has a set of 


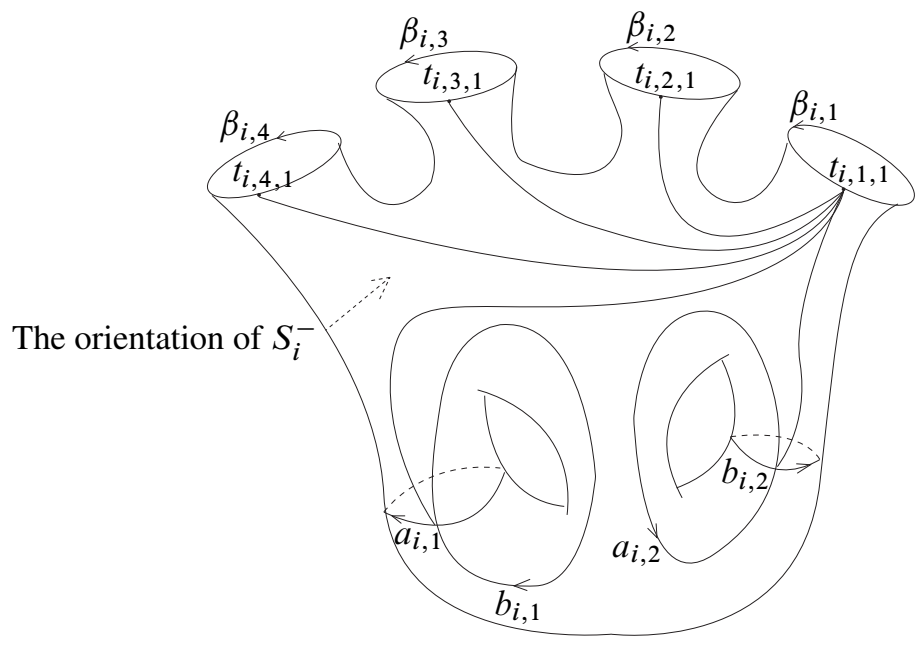

Figure 16: Choice of generators for $\pi_{1}\left(S_{i}^{-}, t_{i, 1,1}\right)$

generators

$$
X=\left\{a_{i, 1}, b_{i, 1}, \ldots, a_{i, g_{i}}, b_{i, g_{i}}, x_{i, 1}, \ldots, x_{i, n_{i}-1}\right\}
$$

such that the elements

$$
x_{i, 1}, x_{i, 2}, \ldots, x_{i, n_{i}-1}, x_{i, n_{i}}=\left[a_{i, 1}, b_{i, 1}\right]\left[a_{i, 2}, b_{i, 2}\right] \cdots\left[a_{i, g_{i}}, b_{i, g_{i}}\right] x_{i, 1} x_{i, 2} \cdots x_{i, n_{i}-1}
$$

have representative loops freely homotopic to the $n_{i}$ boundary components of $S_{i}^{-}$ respectively. In the current case, we pick representative loops based at the point $t_{i, 1,1}$ for the elements $a_{i, 1}, b_{i, 1}, \ldots, a_{i, g_{i}}, b_{i, g_{i}}, x_{i, 1}, \ldots, x_{i, n_{i-1}}$ as shown in Figure 16. For instance $x_{i, 2}$ is represented by the loop which goes along the given arc from $t_{i, 1,1}$ to $t_{i, 2,1}$, then goes around $\beta_{i, 2}$ once following the given orientation and then comes back to $t_{i, 1,1}$ along the given arc from $t_{i, 2,1}$ to $t_{i, 1,1}$. The representative for $x_{i, n_{i}}$ is obtained similarly, except that in this case, we choose a loop which disagrees with the orientation of $\beta_{i, n_{i}}$. Then it is easy to see that

$$
x_{i, n_{i}}=\left[a_{i, 1}, b_{i, 1}\right]\left[a_{i, 2}, b_{i, 2}\right] \cdots\left[a_{i, g_{i}}, b_{i, g_{i}}\right] x_{i, 1} x_{i, 2} \cdots x_{i, n_{i}-1}
$$

is in fact satisfied.

Choice of generators for $\pi_{1}\left(C_{n}\left(J_{i}^{-}\right), b_{i, 1,1}\right)$ Recall from the construction of $C_{n}\left(J_{i}^{-}\right)$ that $C_{n}\left(J_{i}^{-}\right)$is obtained by gluing together $J_{i}^{-}$and $n_{i}$ multi-1-handles $H_{i, 1}^{n}, \ldots, H_{i, n_{i}}^{n}$ along the parabolic regions $D_{i, j, k}, j=1, \ldots, n_{i}, k=1, \ldots, d_{i}$, of $J_{i}^{-}$. Recall that $b_{i, j, k}$ is the center point of $D_{i, j, k}$ which maps to $t_{i, j, k}$. 
Let $\alpha_{i, j, k} \subset J_{i}^{-}$be a fixed, oriented path from $b_{i, 1,1}$ to $b_{i, j, k}, j=1, \ldots, n_{i}, k=$ $1, \ldots, d_{i}\left(\alpha_{i, 1,1}\right.$ is the constant path). For $j=1, \ldots, n_{i}, 1 \leq k \leq d_{i}-1$, let $\delta_{i, j, k}(n)$ be the oriented geodesic arc in $H_{i, j}^{n}$ from $b_{i, j, k}$ to $b_{i, j, k+1}$. For $1 \leq k \leq d_{i}-1$, let $z_{i, j, k}(n)$ be the loop $\alpha_{i, j, k} \cdot \delta_{i, j, k} \cdot \overline{\alpha_{i, j, k+1}}$, where the symbol "." denotes path concatenation (sometimes omitted), and $\overline{\alpha_{i, j, k}}$ denotes the reverse of $\alpha_{i, j, k}$. Also we always write path (in particular loop) concatenation from left to right. We also consider $z_{i, j, k}(n)$ as an element of $\pi_{1}\left(C_{n}\left(J_{i}^{-}\right), b_{i, 1,1}\right)$. Fix a set of generators $w_{i, 1}, \ldots, w_{i, \ell_{i}}$ for $\pi_{1}\left(J_{i}^{-}, b_{i, 1,1}\right)$. Then it's not hard to see, by recalling the structure of $H_{i, j}^{n}$, that $\pi_{1}\left(C_{n}\left(J_{i}^{-}\right), b_{i, 1,1}\right)$ is generated by the set of elements

$$
w_{i, 1}, \ldots, w_{i, \ell_{i}}, z_{i, j, k}(n), 1 \leq j \leq n_{i}, 1 \leq k \leq d_{i}-1 .
$$

In fact

$$
\pi_{1}\left(C_{n}\left(J_{i}^{-}\right), b_{i, 1,1}\right)=\pi_{1}\left(J_{i}^{-}, b_{i, 1,1}\right) *\left\langle z_{i, j, k}(n) \mid 1 \leq j \leq n_{i}, 1 \leq k \leq d_{i}-1\right\rangle,
$$

where $*$ denotes the free product, and $\left\langle z_{i, j, k}(n) \mid 1 \leq j \leq n_{i}, 1 \leq k \leq d_{i}-1\right\rangle$ is the free group freely generated by the $z_{i, j, k}(n)$ 's.

By Lemma 4.2, the local isometry $g_{i}:\left(C_{n}\left(J_{i}^{-}\right), b_{i, 1,1}\right) \rightarrow\left(Y_{i}, t_{i, 1,1}\right)$ induces an injective homomorphism $g_{i}^{*}: \pi_{1}\left(C_{n}\left(J_{i}^{-}\right), b_{i, 1,1}\right) \rightarrow \Gamma_{i}=\pi_{1}\left(Y_{i}, t_{i, 1,1}\right)$. If $\alpha$ is an oriented arc in $C_{n}\left(J_{i}^{-}\right)$, we use $\alpha^{*}$ to denote the oriented arc $g_{i} \circ \alpha$ in $Y_{i}$. We use $\gamma^{*}$ to denote the image of an element $\gamma$ of $\pi_{1}\left(C_{n}\left(J_{i}^{-}\right), b_{i, 1,1}\right)$ under the map $g_{i}^{*}$. Then $g_{i}^{*}\left(\pi_{1}\left(C_{n}\left(J_{i}^{-}\right), b_{i, 1,1}\right)\right)$ is generated by the set of elements

$$
w_{i, 1}^{*}, \ldots, w_{i, \ell_{i}}^{*}, z_{i, j, k}(n)^{*}, 1 \leq j \leq n_{i}, 1 \leq k \leq d_{i}-1 .
$$

Now consider the images of these generators in $Y_{i}$. The oriented path $\alpha_{i, j, k}^{*}$ in $Y_{i}^{-}$ runs from $t_{i, 1,1}$ to $t_{i, j, k}$. For $j=1, \ldots, n_{i}, 1 \leq k \leq d_{i}-1$, let $\eta_{i, j, k}$ be an oriented arc in $\beta_{i, j}$ from $t_{i, j, k}$ to $t_{i, j, k+1}$ following the orientation of $\beta_{i, j}$, and let $\sigma_{i, j, k} \subset Y_{i}^{-}$ be the loop $\alpha_{i, j, k}^{*} \cdot \eta_{i, j, k} \cdot{\overline{\alpha_{i, j, k+1}}}^{*}$. Let $\sigma_{i, j, 0}$ be the constant path based at $t_{i, 1,1}$. Let $x_{i, j}^{\prime}$ be the loop $\alpha_{i, j, 1}^{*} \cdot \beta_{i, j} \cdot{\overline{\alpha_{i, j, 1}}}^{*}$, where $\beta_{i, j}$ is considered an oriented loop starting and ending at the point $t_{i, j, 1}$.

Lemma 10.1 Considered as an element in $\Gamma_{i}$,

$$
z_{i, j, k}(n)^{*}=\left(\overline{\sigma_{i, j, k-1}} \cdots \overline{\sigma_{i, j, 0}}\right)\left(x_{i, j}^{\prime}\right)^{n}\left(\sigma_{i, j, 0} \cdots \sigma_{i, j, k}\right),
$$

for each $j=1, \ldots, n_{i}, k=1, \ldots, d_{i}-1$.

Proof From the construction of $H_{i, j}^{n}$, we see that the $\operatorname{arc} \delta_{i, j, k}(n)^{*}$ is isotopic in $Y_{i}$, with the endpoints fixed, to an arc which: starts from the point $t_{i, j, k}$, goes around $\beta_{i, j}$ exactly $n$ times (following the orientation of $\beta_{i, j}$ ), then continues along $\beta_{i, j}$ 
until it reaches the point $t_{i, j, k+1}$. Now it is easy to check that the loop $z_{i, j, k}(n)^{*}$ is homotopic in $Y_{i}$, fixing the base point $t_{i, 1,1}$, to the loop:

$$
\left(\overline{\sigma_{i, j, k-1}} \cdots \overline{\sigma_{i, j, 0}}\right)\left(x_{i, j}^{\prime}\right)^{n}\left(\sigma_{i, j, 0} \cdots \sigma_{i, j, k}\right)
$$

This proves the lemma.

Remark 10.2 The elements $\sigma_{i, j, k} j=1, \ldots, n_{i}, k=1, \ldots, d_{i}-1$, are independent of the integer $n$.

Definition Suppose that $\breve{p}_{i}: \breve{\beta}_{i, j} \rightarrow \beta_{i, j}$ is a covering map, and let $\breve{\beta}_{i, j}$ have orientation induced from $\beta_{i, j}$. Let $\alpha \subset \widetilde{\beta}_{i, j}$ be an embedded, connected, compact arc with orientation induced from $\breve{\beta}_{i, j}$, whose initial point is in $\breve{p}_{i}^{-1}\left(t_{i, j, k}\right)$ and whose terminal point is in $\breve{p}_{i}^{-1}\left(t_{i, j, k+1}\right)$ (here $k+1$ is defined $\left.\bmod d_{i}\right)$. We say that $\alpha$ has wrapping number $n$ if there are exactly $n$ distinct points of $\breve{p}_{i}^{-1}\left(t_{i, j, k}\right)$ which are contained in the interior of $\alpha$.

In the next section we show the following.

Proposition 10.3 For each $i=1,2$ and $n \geq 0$, there is a finite cover $\breve{Y}_{i}=\breve{S}_{i} \times I$ of $Y_{i}=S_{i} \times I$, having the following properties:

(1) $\partial_{p} \breve{Y}_{i}^{-}=\partial \breve{S}_{i}^{-} \times I$ has the same number of components as $\partial_{p} Y_{i}^{-}=\partial S_{i}^{-} \times I$;

(2) the map $g_{i}: J_{i}^{-} \rightarrow Y_{i}^{-}$lifts to an embedding $\breve{g}_{i}: J_{i}^{-} \rightarrow \breve{Y}_{i}^{-}$;

(3) the points $\breve{g}_{i}\left(b_{i, 1,1}\right), \ldots, \breve{g}_{i}\left(b_{i, n_{i}, d_{i}}\right)$ are evenly spaced; ie there is an integer $N_{i}>n$ such that each of the $n_{i} d_{i}$ components of $\partial \breve{S}_{i}^{-} \backslash\left\{\breve{g}_{i}\left(b_{i, 1,1}\right), \ldots, \breve{g}_{i}\left(b_{i, n_{i}, d_{i}}\right)\right\}$ has wrapping number equal to the integer $N_{i}$.

\section{Adjusting the wrapping numbers}

In this section we prove Proposition 10.3.

Recall from Section 7 that $\widehat{J}_{i}$ is a connected, compact, convex, hyperbolic 3-manifold obtained from $J_{i}^{-}$by capping off each component of $\partial_{p} J_{i}^{-}$with a compact, convex 3ball, and that $\pi_{1}\left(J_{i}, b_{i, 1,1}\right)=\pi_{1}\left(J_{i}^{-}, b_{i, 1,1}\right)=\pi_{1}\left(\widehat{J}_{i}, b_{i, 1,1}\right)$. Also, $\widehat{J}_{i}$ is a submanifold of $C_{n}\left(J_{i}^{-}\right)$, so by Lemma $4.2, \pi_{1}\left(\widehat{J}_{i}, b_{i, 1,1}\right)$ can be considered as a subgroup of $\pi_{1}\left(C_{n}\left(J_{i}^{-}\right), b_{i, 1,1}\right)$.

By Proposition 4.7 there is a set of elements $y_{i, 1}, \ldots, y_{i, r_{i}}$ in $\Gamma_{i}-g_{i}^{*}\left(\pi_{1}\left(\widehat{J}_{i}, b_{i, 1,1}\right)\right)$ such that, if $G_{i}$ is a finite index subgroup of $\Gamma_{i}$ which separates $g_{i}^{*}\left(\pi_{1}\left(\widehat{J}_{i}, b_{i, 1,1}\right)\right)$ 
from $y_{i, 1}, \ldots, y_{i, r_{i}}$, then the local isometry $g_{i}: \widehat{J}_{i} \rightarrow Y_{i}$ lifts to an embedding $\breve{g}_{i}$ in the finite cover $\breve{Y}_{i}$ corresponding to $G_{i}$.

To prove Proposition 10.3, we shall construct a finite index subgroup $G_{i}$ of $\Gamma_{i}$, of sufficiently large index $m_{i}$, such that:

(i) $m_{i}=N_{i} d_{i}+1$ for some integer $N_{i}>n$;

(ii) $G_{i}$ contains the elements $w_{i, 1}^{*}, \ldots, w_{i, \ell_{i}}^{*}$ (defined in Section 10), and thus contains the subgroup $g_{i}^{*}\left(\pi_{1}\left(\widehat{J}_{i}, b_{i, 1,1}\right)\right)$;

(iii) $G_{i}$ contains the elements $z_{i, j, k}\left(N_{i}\right)^{*}, j=1, \ldots, n_{i}, k=1, \ldots, d_{i}-1$;

(iv) $G_{i}$ does not contain any of $x_{i, j}^{l}, j=1, \ldots, n_{i}$, and $l=1, \ldots, m_{i}-1$;

(v) $G_{i}$ does not contain any of $y_{i, 1}, \ldots, y_{i, r_{i}}$.

Proposition 11.1 Assuming such a subgroup $G_{i}$ can be found, then the corresponding finite cover $\breve{Y}_{i}=X_{i} / G_{i}$ of $Y_{i}$ will satisfy all the properties given in Proposition 10.3.

Proof Suppose that $\breve{q}_{i}: X_{i} \rightarrow \breve{Y}_{i}$ and $\breve{p}_{i}: \breve{Y}_{i} \rightarrow Y_{i}$ are the covering maps. Properties (ii) and (v) imply that the map $g_{i}:\left(\widehat{J}_{i}, b_{i, 1,1}\right) \rightarrow\left(Y_{i}, t_{i, 1,1}\right)$ lifts to an embedding $\breve{g}_{i}:\left(\widehat{J}_{i}, b_{i, 1,1}\right) \rightarrow\left(\breve{Y}_{i}, \breve{q}_{i}(\tilde{b})\right)$. By our choice for the cusp $C$ of $M$ (which determines the cusp region $\mathcal{C}_{i}$ for $Y_{i}$ ), the restriction of $\breve{g}_{i}$ on $J_{i}^{-}$gives a proper embedding $\breve{g}_{i}:\left(J_{i}^{-}, \partial J_{i}^{-}\right) \rightarrow\left(\breve{Y}_{i}^{-}, \partial \breve{Y}_{i}^{-}\right)$, ie we have (2) of Proposition 10.3.

We claim that condition (iv) implies $\partial_{p} \breve{Y}_{i}^{-}$has the same number of components as $\partial_{p} Y_{i}^{-}$, ie we have (1) of Proposition 10.3. To see this, recall that each of $x_{i, j}$, $j=1, \ldots, n_{i}$, has a representative loop (Figure 16) which is homotopic, with the base point $t_{i, 1,1}$ fixed, to an embedded loop, $x_{i, j}^{\prime \prime}$, in $S_{i}^{-}$, such that $x_{i, j}^{\prime \prime}$ is parallel to $\beta_{i, j}$ in $S_{i}^{-}$. Since $G_{i}$ does not contain any of the elements $x_{i, j}^{l}, j=1, \ldots, n_{i}$, $l=1, \ldots, m_{i}-1$, then $\breve{p}_{i}^{-1}\left(x_{i, j}^{\prime \prime}\right)$ is a single, embedded loop in $\breve{S}_{i}^{-}$for each fixed $j=1, \ldots, n_{i}$. Hence $\breve{p}_{i}^{-1}\left(\beta_{i, j}\right)$ is a single component of $\partial \breve{S}_{i}^{-}$for each $j=1, \ldots, n_{i}$. This proves the claim.

We now show that condition (i) and (iii) imply (3) of Proposition 10.3. Namely we want to show that the set of points $\breve{g}_{i}\left(b_{i, 1,1}\right), \ldots, \breve{g}_{i}\left(b_{i, n_{i}, d_{i}}\right)$ are evenly spaced in $\partial \breve{S}_{i}^{-}$so that each of the $n_{i} d_{i}$ components of $\partial \breve{S}_{i}^{-} \backslash\left\{\breve{g}_{i}\left(b_{i, 1,1}\right), \ldots, \breve{g}_{i}\left(b_{i, n_{i}, d_{i}}\right)\right\}$ has wrapping number equal to $N_{i}$.

Consider the manifold $C_{N_{i}}\left(J_{i}^{-}\right)$. As noted in the previous section, the subgroup

$$
g_{i, N_{i}}^{*}\left(\pi_{1}\left(C_{N_{i}}\left(J_{i}^{-}\right), b_{i, 1,1}\right)\right) \subset \Gamma_{i}
$$


is generated by the elements $w_{i, 1}^{*}, \ldots, w_{i, \ell_{i}}^{*}$ and $z_{i, j, k}\left(N_{i}\right)^{*}, j=1, \ldots, n_{i}, k=$ $1, \ldots, d_{i}-1$. Hence the group $g_{i, N_{i}}^{*}\left(\pi_{1}\left(C_{N_{i}}\left(J_{i}^{-}\right), b_{i, 1,1}\right)\right)$ is contained in $G_{i}$ by conditions (ii) and (iii). Therefore the map $g_{i}:\left(C_{N_{i}}\left(J_{i}^{-}\right), b_{i, 1,1}\right) \rightarrow\left(Y_{i}, t_{i, 1,1}\right)$ lifts to a map $\breve{g}_{i}:\left(C_{N_{i}}\left(J_{i}^{-}\right), b_{i, 1,1}\right) \rightarrow\left(\breve{Y}_{i}, \breve{q}_{i}(\tilde{b})\right)$, ie $\breve{p}_{i} \circ \breve{g}_{i}=g_{i}$.

Let $\breve{\beta}_{i, j}$ be the component of $\partial \breve{S}_{i}^{-}$which covers $\beta_{i, j}, j=1, \ldots, n_{i}$. Then by (1) and condition (i), $\breve{p}_{i}: \breve{\beta}_{i, j} \rightarrow \beta_{i, j}$ is an $N_{i} d_{i}+1$-fold cyclic covering, for each $j=$ $1, \ldots, n_{i}$. For each fixed $j=1, \ldots, n_{i}$, the set of points $\left\{\breve{g}_{i, N_{i}}\left(b_{i, j, k}\right), k=1, \ldots, d_{i}\right\}$ divides $\breve{\beta}_{i, j}$ into $d_{i}$ segments.

Recall the notations established in Section 10. Consider the multi-handle $H_{i, j}^{N_{i}} \subset$ $C_{N_{i}}\left(J_{i}^{-}\right)$containing the points $b_{i, j, 1}, \ldots b_{i, j, d_{i}}$, and the geodesic $\operatorname{arcs} \delta_{i, j, k}\left(N_{i}\right) \subset$ $H_{i, j}^{N_{i}}, k=1, \ldots, d_{i}-1$. By our construction the immersed arc $g_{i, N_{i}}: \delta_{i, j, k}\left(N_{i}\right) \rightarrow$ $S_{i}$ is homotopic, with end points fixed, to the arc in $\beta_{i, j}$ which starts at the point $t_{i, j, k}$, wraps $N_{i}$ times around $\beta_{i, j}$ and then continues to the point $t_{i, j, k+1}$, following the orientation of $\beta_{i, j}$. This latter (immersed) arc lifts to an embedded arc in $\breve{\beta}_{i, j}$ connecting $\breve{g}_{i, N_{i}}\left(b_{i, j, k}\right)$ and $\breve{g}_{i, N_{i}}\left(b_{i, j, k+1}\right)$, since $\breve{\beta}_{i, j}$ is an $N_{i} d_{i}+1$-fold cyclic cover of $\beta_{i, j}$. Now it is easy to see that the conclusion of (3) follows.

To find the required subgroup $G_{i}$ of $\Gamma_{i}$, we apply again the graph technique used in Section 9. We shall use terminologies established there without recalling them again. From now on all elements of $\Gamma_{i}$ will be considered as words in letters from $L_{i} \cup L_{i}^{-1}$, where

$$
L_{i}=\left\{a_{i, 1}, b_{i, 1}, \ldots, a_{i, g_{i}}, b_{i, g_{i}}, x_{i, 1}, \ldots, x_{i, n_{i}-1}\right\}
$$

is the free basis of $\Gamma_{i}$ given in Section 10. For simplicity a word $w$ in letters of $L_{i} \cup L_{i}^{-1}$ shall also be considered as a path in a $L_{i}$-labeled directed graph, and the context will make it clear which is meant.

From Section 9 we know that to find the required subgroup $G_{i}$ of $\Gamma_{i}$, it suffices to find a finite, connected, $L_{i}$-labeled, directed graph $\mathcal{G}_{i}$ (with a fixed base vertex $v_{i, 0}$ ) with the following properties:

(0) $\mathcal{G}_{i}$ is $L_{i}$-regular;

(1) $m_{i}=N_{i} d_{i}+1$ for some integer $N_{i}>n$, where $m_{i}$ is the number of vertices of $\mathcal{G}_{i}$

(2) each of the words $w_{i, 1}^{*}, \ldots, w_{i, \ell_{i}}^{*}$ is representable by a loop, based at $v_{i, 0}$, in $\mathcal{G}_{i}$;

(3) $\mathcal{G}_{i}$ contains a closed loop, based at $v_{i, 0}$, representing the word $z_{i, j, k}\left(N_{i}\right)^{*}$, for each $j=1, \ldots, n_{i}, k=1, \ldots, d_{i}-1$; 
(4) $\mathcal{G}_{i}$ contains no loop representing the word $x_{i, j}^{l}$ for any $j=1, \ldots, n_{i}$ and $l=$ $1, \ldots, m_{i}-1$;

(5) each of the words $y_{i, 1}, \ldots, y_{i, r_{i}}$ is representable by a non-closed path, based at $v_{i, 0}$, in $\mathcal{G}_{i}$.

If such a graph can be found, then the subgroup of $\Gamma_{i}$ represented by $L\left(\mathcal{G}_{i}, v_{i, 0}\right)$ will satisfy all the requirements (i)-(v) set for $G_{i}$. Indeed, Properties (0) and (1) of $\mathcal{G}_{i}$ imply Property (i) of $G_{i}$ (Lemma 9.3), and Properties (2)-(5) of $\mathcal{G}_{i}$ imply Properties (ii)-(v) of $G_{i}$ respectively. The task of the rest of this section is to construct such a graph $\mathcal{G}_{i}$.

If $\mathcal{G}$ is an $L_{i}$-labeled directed graph, then $\mathcal{G}^{f}$ will denote the folded graph resulting from folding $\mathcal{G}$ (see Kapovich and Myasnikov [11] for the folding operation). Note that if $\mathcal{G}$ is an $L_{i}$-labeled directed graph, and $\mathcal{G}^{\prime}$ is a graph obtained from $\mathcal{G}$ by performing some folding operations on $\mathcal{G}$, then there is a uniquely associated quotient map $q: \mathcal{G} \rightarrow \mathcal{G}^{\prime}$. In particular there is a uniquely associated quotient map from $\mathcal{G}$ to $\mathcal{G}^{f}$.

Let $n$ be a large integer such that the manifold $C_{n}\left(J_{i}^{-}\right)$is convex, for each of $i=1,2$. Hence the local isometry $g_{i}: C_{n}\left(J_{i}^{-}\right) \rightarrow Y_{i}$ induces an injective homomorphism $g_{i}^{*}: \pi_{1}\left(C_{n}\left(J_{i}^{-}\right), b_{i, 1,1}\right) \rightarrow \pi_{1}\left(Y_{i}, t_{i, 1,1}\right)$.

Recall that the subgroup $g_{i}^{*}\left(\pi_{1}\left(C_{n}\left(J_{i}^{-}\right), b_{i, 1,1}\right) \subset \Gamma_{i}\right.$ is generated by the elements

$$
w_{i, 1}^{*}, \ldots, w_{i, \ell_{i}}^{*}, z_{i, j, k}(n)^{*}, 1 \leq j \leq n_{i}, 1 \leq k \leq d_{i}-1 .
$$

Let $\mathcal{G}_{i, 0}(n)$ be the connected, finite, $L_{i}$-labeled, directed graph which results from taking a disjoint union of embedded loops, representing the reduced versions of the words

$$
w_{i, 1}^{*}, \ldots, w_{i, \ell_{i}}^{*}, z_{i, j, k}(n)^{*}, 1 \leq j \leq n_{i}, 1 \leq k \leq d_{i}-1
$$

respectively, and non-closed embedded paths, representing the reduced versions of the words

$$
y_{i, 1}, \ldots, y_{i, r_{i}}
$$

respectively, and then identifying their base vertices (their initial vertices) to a common vertex $v_{i, 0}$.

Then obviously $L\left(\mathcal{G}_{i, 0}(n), v_{i, 0}\right)=L\left(\mathcal{G}_{i, 0}(n)^{f}, v_{i, 0}\right)=g_{i}^{*}\left(\pi_{1}\left(C_{n}\left(J_{i}^{-}\right), b_{i, 1,1}\right)\right)$.

We may consider a graph $\mathcal{G}$ as metric space, by making each edge isometric to the interval $[0,1]$, and taking the induced path metric. If $x \in \mathcal{G}$ and $s \in \mathbb{R}$, then $N_{S}(x)$ denotes the $s$-neighborhood of $x$ in $\mathcal{G}$.

Lemma 11.2 There is an integer $s>0$, independent of $n$, such that, when $n$ is large, the natural quotient map $f: \mathcal{G}_{i, 0}(n) \rightarrow \mathcal{G}_{i, 0}(n)^{f}$ is an embedding on $\mathcal{G}_{i, 0} \backslash N_{s}\left(v_{i, 0}\right)$, and each of $f\left(y_{i, 1}\right), \ldots, f\left(y_{i, r_{i}}\right)$ is still a non-closed path in $\mathcal{G}_{i, 0}(n)^{f}$. 
Proof We give an explicit construction of $\mathcal{G}_{i, 0}(n)^{f}$, building it in steps.

Let $\mathcal{G}_{i, 1}$ be the connected, finite, $L_{i}$-labeled, directed graph which results from taking a disjoint union of embedded loops- representing the reduced versions of the words $w_{i, 1}^{*}, \ldots, w_{i, \ell_{i}}^{*}$ respectively- and non-closed embedded paths- representing the reduced versions of the words $y_{i, 1}, \ldots, y_{i, r_{i}}$ respectively- and then identifying their base points to a common vertex $v_{i, 0}$. Then obviously $L\left(\mathcal{G}_{i, 1}, v_{i, 0}\right)$ represents the subgroup $g_{i}^{*}\left(\pi_{1}\left(J_{i}^{-}, b_{i, 1,1}\right)\right) \subset \Gamma_{i}$. Since the folding operation does not change the group that the graph represents, $L\left(\mathcal{G}_{i, 1}^{f}, v_{i, 0}\right)=g_{i}^{*}\left(\pi_{1}\left(J_{i}^{-}, b_{i, 1,1}\right)\right)$. By assumption, none of the elements $y_{i, 1}, \ldots, y_{i, r_{i}}$ belong to the subgroup $g_{i}^{*}\left(\pi_{1}\left(J_{i}^{-}, b_{i, 1,1}\right)\right)$, so $y_{i, 1}, \ldots, y_{i, r_{i}}$ are still non-closed paths in $\mathcal{G}_{i, 1}^{f}$ based at $v_{i, 0}$.

Recall from Lemma 10.1 that

$$
z_{i, j, k}(n)^{*}=\left(\overline{\sigma_{i, j, k-1}} \cdots \overline{\sigma_{i, j, 0}}\right)\left(x_{i, j}^{\prime}\right)^{n}\left(\sigma_{i, j, 0} \cdots \sigma_{i, j, k}\right)
$$

for $k=1, \ldots, d_{i}-1$, where $\sigma_{i, j, k}$ and $x_{i, j}^{\prime}$ were defined in Section 8. Note that $x_{i, j}^{\prime}$ is conjugate to $x_{i, j}$ in $\Gamma_{i}$. Let $\tau_{i, j}$ be an element of $\Gamma_{i}$ such that $x_{i, j}^{\prime}=\tau_{i, j} x_{i, j} \tau_{i, j}^{-1}$. Let $\mathcal{G}_{i, 2}$ be the connected graph which results from taking the disjoint union of $\mathcal{G}_{i, 1}^{f}$ and non-closed embedded paths representing the reduced version of the words $\bar{\sigma}_{i, j, k-1} \cdots \bar{\sigma}_{i, j, 0} \tau_{i, j}, 1 \leq j \leq n_{i}, 1 \leq k \leq d_{i}$, respectively, and then identifying their base vertices into a single base vertex which we still denote by $v_{i, 0}$. Then obviously we have $L\left(\mathcal{G}_{i, 2}^{f}, v_{i, 0}\right)=L\left(\mathcal{G}_{i, 2}, v_{i, 0}\right)=L\left(\mathcal{G}_{i, 1}^{f}, v_{i, 0}\right)=g_{i}^{*}\left(\pi_{1}\left(J_{i}^{-}, b_{i, 1,1}\right)\right)$.

Let $v_{i, j, k}$ be the terminal vertex of the path $\overline{\sigma_{i, j, k-1}} \ldots \overline{\sigma_{i, j, 0}} \tau_{i, j}$ in $\mathcal{G}_{i, 2}^{f}$, for each $j=1, \ldots, n_{i}$ and $k=1, \ldots, d_{i}$. For each $j=1, \ldots, n_{i}-1$ (when $\left.n_{i}>1\right)$ and $k=1, \ldots, d_{i}$, let $q_{i, j, k}$ be the maximal $x_{i, j}$-path in $\widehat{\mathcal{G}_{i, 2}^{f}}$ (a maximal $x_{i, j}$-path was defined in Section 9) which contains the vertex $v_{i, j, k}$. For $j=n_{i}$, and each $k=1, \ldots, d_{i}$, let $q_{i, n_{i}, k}$ be the maximal $x_{i, n_{i}}$-path in $\widehat{\mathcal{G}_{i, 2}^{f}}$ determined by:

(1) if there is a directed edge of $\widehat{\mathcal{G}_{i, 2}^{f}}$ with $v_{i, j, k}$ as its initial vertex and with the first letter of the word $x_{i, n_{i}}$ as its label, then $q_{i, n_{i}, k}$ contains that edge;

(2) if the edge described in (1) does not exist, then $v_{i, j, k}$ is the terminal vertex of $q_{i, n_{i}, k}$ and the first letter of the word $x_{i, n_{i}}$ is the terminal missing label of $q_{i, n_{i}, k}$.

Note that each $q_{i, j, k}$ is uniquely determined. Also no $q_{i, j, k}$ can be an $x_{i, j}$-loop, since the group $L\left(\mathcal{G}_{i, 2}^{f}, v_{i, 0}\right)=g_{i}^{*}\left(\pi_{1}\left(J_{i}^{-}, b_{i, 1,1}\right)\right)$ does not contain non-trivial peripheral elements of $\Gamma_{i}$. Let $v_{i, j, k}^{-}$and $v_{i, j, k}^{+}$be the initial and terminal vertices of $q_{i, j, k}$ respectively. Note that if $j<n_{i}$ and $q_{i, j, k}$ is not a constant path, then $v_{i, j, k}^{-}$and $v_{i, j, k}^{+}$ 
must be distinct vertices; however $v_{i, n_{i}, k}^{-}$and $v_{i, n_{i}, k}^{+}$may possibly be the same vertex, even if $q_{i, j, k}$ is a non-constant path.

For each $j=1, \ldots, n_{i}$ and $k=1, \ldots, d_{i}$, let $q_{i, j, k}^{-}$be the embedded subpath of $q_{i, j, k}$ with $v_{i, j, k}^{-}$as the initial vertex and with $v_{i, j, k}$ as the terminal vertex, and let $q_{i, j, k}^{+}$be the embedded subpath of $q_{i, j, k}$ with $v_{i, j, k}$ as the initial vertex and with $v_{i, j, k}^{+}$as the terminal vertex.

Note that the set $\left\{\right.$ Length $\left.\left(q_{i, j, k}\right): i=1,2, j=1, \ldots, n_{i}, k=1, \ldots, d_{i}\right\}$ is independent of $n$, and thus is bounded. So we may assume that for each $i=1,2$,

$$
n>10+\max \left\{2 \operatorname{Length}\left(q_{i, j, k}\right): i=1,2, j=1, \ldots, n_{i}, k=1, \ldots, d_{i}\right\} .
$$

Now for each $j=1, \ldots, n_{i}$ and $k=1, \ldots, d_{i}-1$, we make a new non-closed embedded path $\Theta_{i, j, k}(n)$ representing the word $x_{i, j}^{n}$, and we add it to the graph $\mathcal{G}_{i, 2}^{f}$, by identifying the initial vertex of $\Theta_{i, j, k}(n)$ with $v_{i, j, k}$ and the terminal vertex with $v_{i, j, k+1}$. In the resulting graph there are some obvious places one can perform the folding operation: for each $j=1, \ldots, n_{i}$ and $k=1, \ldots, d_{i}-1$, the path $q_{i, j, k}^{+}$can be completely folded into the added new path $\Theta_{i, j, k}(n)$, and likewise the path $q_{i, j, k+1}^{-}$can be completely folded into $\Theta_{i, j, k}(n)$. Let $\mathcal{G}_{i, 3}(n)$ be the resulting graph after performing these specific folding operations for each $j=1, \ldots, n_{i}$ and $k=1, \ldots, d_{i}-1$.

From the explicit construction, it is clear that $\mathcal{G}_{i, 3}(n)$ has the following properties:

(1) $\mathcal{G}_{i, 3}(n)$ is a connected, finite, $L_{i}$-labeled, directed graph;

(2) $\mathcal{G}_{i, 3}(n)$ contains loops, based at $v_{i, 0}$, representing the word $z_{i, j, k}(n)^{*}$ for each $j=1, \ldots, n_{i}, k=1, \ldots, d_{i}-1$;

(3) $\mathcal{G}_{i, 3}(n)$ contains $\mathcal{G}_{i, 2}^{f}$ as an embedded subgraph;

(4) $\mathcal{G}_{i, 3}(n)$ is obtained from $\mathcal{G}_{i, 0}(n)$ by a sequence of folds.

It follows from Property (3) that the paths in $\mathcal{G}_{i, 2}^{f}$ representing the words $y_{i, 1}, \ldots, y_{i, r_{i}}$ remain each non-closed in $\mathcal{G}_{i, 3}(n)$, and it follows from Property (4) that:

$$
L\left(\mathcal{G}_{i, 3}(n), v_{i, 0}\right)=L\left(\mathcal{G}_{i, 0}(n), v_{i, 0}\right)=g_{i}^{*}\left(\pi_{1}\left(C_{n}\left(J_{i}^{-}\right), b_{i, 1,1}\right)\right) .
$$

So $\widehat{\mathcal{G}_{i, 3}(n)}$ cannot have $x_{i, j}$-loops for any $j$.

Now we consider the remaining folding operations on $\mathcal{G}_{i, 3}(n)$ that need to be done, in order to get the folded graph $\mathcal{G}_{i, 3}(n)^{f}$.

For each $j=1, \ldots, n_{i}$ and $k=1, \ldots, d_{i}-1$, let $\Theta_{i, j, k}(n)^{\prime}=\Theta_{i, j, k}(n) \backslash\left(q_{i, j, k}^{+} \cup\right.$ $\left.q_{i, j, k+1}^{-}\right)$. Then by our construction each $\Theta_{i, j, k}(n)^{\prime}$ is an embedded $x_{i, j}$-path with 
$v_{i, j, k}^{+}$as its initial vertex and with $v_{i, j, k+1}^{-}$as the terminal vertex, and contains a subpath representing the word $x_{i, j}^{10}$. Also all these paths $\Theta_{i, j, k}(n)^{\prime}, j=1, \ldots, n_{i}$ and $k=1, \ldots, d_{i}-1$, are mutually disjoint in their interior, and their disjoint union is equal to $\mathcal{G}_{i, 3}(n) \backslash \mathcal{G}_{i, 2}^{f}$.

For each fixed $j=1, \ldots, n_{1}$, there is an $x_{i, j}$-path in $\mathcal{G}_{i, 3}(n)$ with $v_{i, j, 1}^{-}$as the initial vertex and with $v_{i, j, d_{i}}^{+}$as the terminal vertex, containing all the vertices $v_{i, j, k}^{ \pm}$, $k=1, \ldots, d_{i}$, and containing all the paths $\Theta_{i, j, k}(n), k=1, \ldots, d_{i}-1$. Since $\widehat{\mathcal{G}_{i, 3}(n)}$ has no $x_{i, j}$-loops, we see immediately that when $j<n_{i}$, all the vertices $v_{i, j, k}^{ \pm}, k=1, \ldots, d_{i}$, are mutually distinct.

We know that:

(1) each vertex $v_{i, j, k}^{ \pm}$is a initial or terminal vertex of a maximal $x_{i, j}$-path in $\mathcal{G}_{i, 2}^{f}$;

(2) the graph $\mathcal{G}_{i, 2}^{f}$ is an embedded, folded subgraph of $\mathcal{G}_{i, 3}(n)$;

(3) each $\Theta_{i, j, k}(n)^{\prime}$ is an embedded path in $\mathcal{G}_{i, 3}(n)$;

(4) for each fixed $j<n_{i}$, all the vertices $v_{i, j, k}^{ \pm}, k=1, \ldots, d_{i}$, are mutually distinct. It follows that the only remaining folds are at the vertices $v_{i, n_{i}, k}^{ \pm}$, where possibly a single edge from $\Theta_{i, n_{i}, k}(n)^{\prime}$ may be folded to a single edge from $\Theta_{i, j, k_{*}}(n)^{\prime}$, for some $1 \leq j<n_{i}$ and some $1 \leq k_{*} \leq d_{i}-1$. At such a vertex there is at most one edge from $\Theta_{i, n_{i}, k}(n)^{\prime}$ which may be folded with one $x_{i, j}$-edge of $\Theta_{i, j, k_{*}}(n)^{\prime}$. Thus $\mathcal{G}_{i, 3}(n)^{f}$ is obtained from $\mathcal{G}_{i, 3}(n)$ by performing at most $2 d_{i}$ folds (which occur at some of the vertices $\left.v_{i, n_{i}, k}^{ \pm}, k=1, \ldots, d_{i}\right)$, and every non-closed, reduced path in $\mathcal{G}_{i, 3}(n)$ which is based at $v_{i, 0}$ will remain non-closed in $\mathcal{G}_{i, 3}(n)^{f}$. In particular, the paths representing the words $y_{i, 1}, \ldots y_{i, r_{i}}$ are each non-closed in $\mathcal{G}_{i, 3}(n)^{f}=\mathcal{G}_{i, 0}(n)^{f}$.

Let $f_{3}: \mathcal{G}_{i, 3}(n) \rightarrow \mathcal{G}_{i, 3}(n)^{f}$ be the natural map. Then by the construction, we see that if $s_{1}$ is greater than $2 d_{i}+\operatorname{Diameter}\left(\mathcal{G}_{i, 2}^{f}\right)$, then the map $f_{3}: \mathcal{G}_{i, 3}(n) \rightarrow \mathcal{G}_{i, 3}(n)^{f}$ is an embedding on $\mathcal{G}_{i, 3}(n)-N_{S_{1}}\left(v_{i, 0}\right)$. Since $\mathcal{G}_{i, 3}(n)$ is a partial folding of $\mathcal{G}_{i, 0}(n)$, there is a quotient map $g: \mathcal{G}_{i, 0}(n) \rightarrow \mathcal{G}_{i, 3}(n)$. Letting $s$ be the diameter of $g^{-1}\left(N_{s_{1}}\left(v_{i, 0}\right)\right)$, then $g$ is an embedding on $\mathcal{G}_{i, 0}(n)-N_{s}\left(v_{i, 0}\right)$. Since the map $f: \mathcal{G}_{i, 0}(n) \rightarrow \mathcal{G}_{i, 0}(n)^{f}=$ $\mathcal{G}_{i, 3}(n)^{f}$ is the composition of the maps $g$ and $f_{3}$, we see that $f$ is an embedding on $\mathcal{G}_{i, 0}-N_{s}\left(v_{i, 0}\right)$. Obviously the number $s$ is independent of $n$. The proof of Lemma 11.2 is now complete.

Let $s$ be the constant integer guaranteed by Lemma 11.2. We may assume that $s$ is large enough so that $N_{s}\left(v_{i, 0}\right)$ in $\mathcal{G}_{i, 0}(n)$ contains the loops $w_{i, 1}^{*}, \ldots, w_{i, \ell_{i}}^{*}$, the paths $y_{1}, \ldots, y_{r_{i}}$ and the paths representing the words $\overline{\sigma_{i, j, k-1}} \cdots \overline{\sigma_{i, j, 0}} \tau_{i, j}, j=1, \ldots, n_{i}$, 
$k=1, \ldots, d_{i}-1$. (The choice of $s$ given in the proof of Lemma 11.2 actually already satisfies this requirement.) We may assume further that $n$ is large enough so that the components of $\mathcal{G}_{i, 0}(n)^{f} \backslash f\left(N_{v_{i, 0}}(s)\right)$ can be denoted by $\Phi_{i, j, k}(n), 1 \leq j \leq n_{i}$, $1 \leq k \leq d_{i}-1$, such that $\Phi_{i, j, k}(n)$ is an embedded subpath in $\Theta_{i, j, k}(n)^{\prime}$ (and thus is a $x_{i, j}$-path) containing a sufficiently large power of $x_{i, j}$. This is clearly possible from the proof of Lemma 11.2.

The next step is to modify the graph $\mathcal{G}_{i, 0}(n)^{f}$, by inserting copies of a certain graph $\Omega$, pictured in Figure 17, and then performing folding operations, to obtain a graph (the graph $\mathcal{G}_{i, 4}(n)$ given below) which contains loops, based at the base vertex $v_{i, 0}$, representing the words

$$
w_{i, 1}^{*}, \ldots, w_{i, \ell_{i}}^{*}, z_{i, j, k}(n+1)^{*}, 1 \leq j \leq n_{i}, 1 \leq k \leq d_{i}-1,
$$

respectively, and which contains non-closed paths, based at $v_{i, 0}$, representing the words

$$
y_{i, 1}, \ldots, y_{i, r_{i}}
$$

respectively. Then from this graph we can go two steps further to find the required graph (The graph $\mathcal{G}_{i, 6}(n)$ given afterwards). The method for constructing $\mathcal{G}_{i, 4}(n)$ breaks into three cases, ie

(a) when $n_{i}$ is even,

(b) when $n_{i}>1$ is odd, and

(c) when $n_{i}=1$.

In Figure 17, single edge loops at a vertex have one label each from the labels $L_{i}^{*}=$ $\left\{a_{i, 1}, b_{i, 1}, \ldots, a_{i, g_{i}}, b_{i, g_{i}}\right\}$. The edges in part (a) and (b) connecting two adjacent vertices are $x_{i, j}$-edges, $j=1,2, \ldots, n_{i}-1$, (precisely $n_{i}--1$ edges). In part (a) of the figure, an $x_{i, j}$-edge points from the left vertex to the right vertex iff $j$ is odd, and in part (b) of the figure, an $x_{i, j}$-edge points from left to right iff $j$ is 1 or an even number. The edges in part (c) connecting the left two vertices and pointing from left to right are labeled $a_{i, j}$ and $b_{i, j}, j=1,2, \ldots, g_{i}$, respectively, while the edges connecting the left two vertices but pointing from right to left are labeled $b_{i, 1}, a_{i, j}$, $b_{i, j}, j=2, \ldots, g_{i}$, respectively. The right half of (c) is an identical copy of the left half of (c).

Case (a) $n_{i}$ is even.

We shall insert $d_{i}-1$ copies of the graph $\Omega$ (Figure 17 part (a)), denoted $\Omega_{k}, k=$ $1, \ldots, d_{i}-1$, as follows. For each $1 \leq k \leq d_{i}-1$, we define a subset of vertices $\mathcal{U}_{i, k}=\left\{u_{i, j, k}: 1 \leq j \leq n_{i}\right\} \subset \mathcal{G}_{i, 0}(n)^{f}$ where,

- if $j \leq n_{i}-1$, then $u_{i, j, k}$ is a vertex in $\Phi_{i, j, k}(n)$, such that there are at least three 


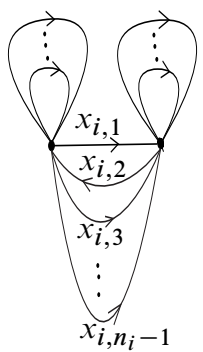

(a)

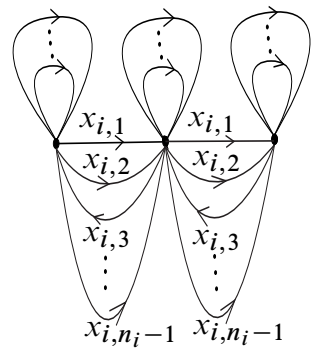

(b)

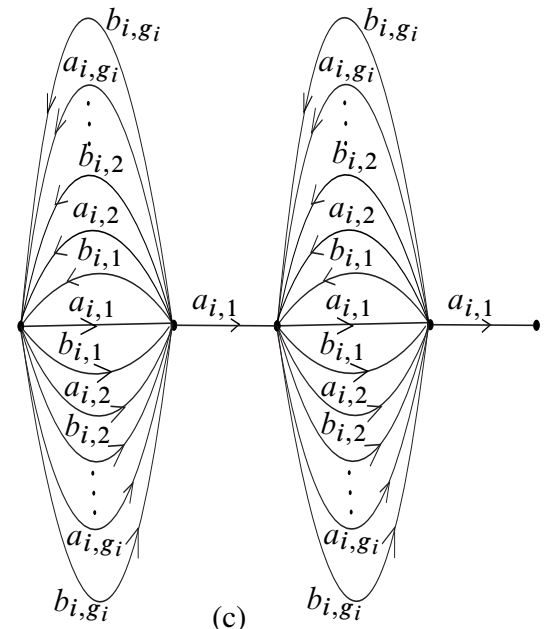

(c)

Figure 17: The graph $\Omega$ when: (a) $n_{i}$ is even (b) $n_{i}>1$ is odd (c) $n_{i}=1$

edges before it and after it in the directed (and thereby ordered) path $\Phi_{i, j, k}(n)$, and - $u_{i, n_{i}, k}$ is the initial vertex of an edge labeled $x_{1}$ in $\Phi_{i, n_{i}, k}(n)$ such that there are at least three edges with label $x_{1}$ before it and after it in the path $\Phi_{i, n_{i}, k}(n)$.

Then cut $\mathcal{G}_{i, 0}(n)^{f}$ at the vertices of $\mathcal{U}_{i, k}, k=1, \ldots, d_{i}-1$, and for each $k$, insert the graph $\Omega_{k}$, which is a copy of the graph $\Omega$ shown in Figure 17 (a). That is, we:

(1) Form a cut graph $\mathcal{G}_{i, 0}(n)_{c}^{f}=\mathcal{G}_{i, 0}(n)^{f} \backslash\left\{U_{i, k} ; k=1, \ldots, d_{i}-1\right\}$, whose vertex set is obtained from the vertex set of $\mathcal{G}_{i, 0}(n)^{f}$ by replacing each $u_{i, j, k} \in \mathcal{U}_{i, k}$ with a pair of vertices $u_{i, j, k}^{ \pm}$. More precisely the point $u_{i, j, k}$ cuts the path $\Phi_{i, j, k}$ into two components; $u_{i, j, k}^{+}$is the terminal vertex of one component, and $u_{i, j, k}^{-}$is the initial vertex of the other component. If each pair $\left\{u_{i, j, k}^{+}, u_{i, j, k}^{-}\right\}$is identified into a single vertex, then the resulting graph is $\mathcal{G}_{i, 0}(n)^{f}$.

(2) For each fixed $k=1, \ldots, d_{i}-1$, we identify the vertex set $\left\{u_{i, j, k}^{ \pm}, j=1, \ldots, n_{i}\right\}$ of $\mathcal{G}_{i, 0}(n)_{c}^{f}$ with the vertices of $\Omega_{k}$ as follows:

- if $j<n_{i}$, identify $u_{i, j, k}^{+}$with the left vertex of $\Omega_{k}$ if $j$ is odd and to the right vertex if $j$ is even, and identify $u_{i, j, k}^{-}$with the right vertex of $\Omega_{k}$ if $j$ is odd and to the left vertex if $j$ is even;

- identify $u_{i, n_{i}, k}^{+}$with the left vertex of $\Omega_{k}$ and identify $u_{i, n_{i}, k}^{-}$with the right vertex of $\Omega_{k}$. 


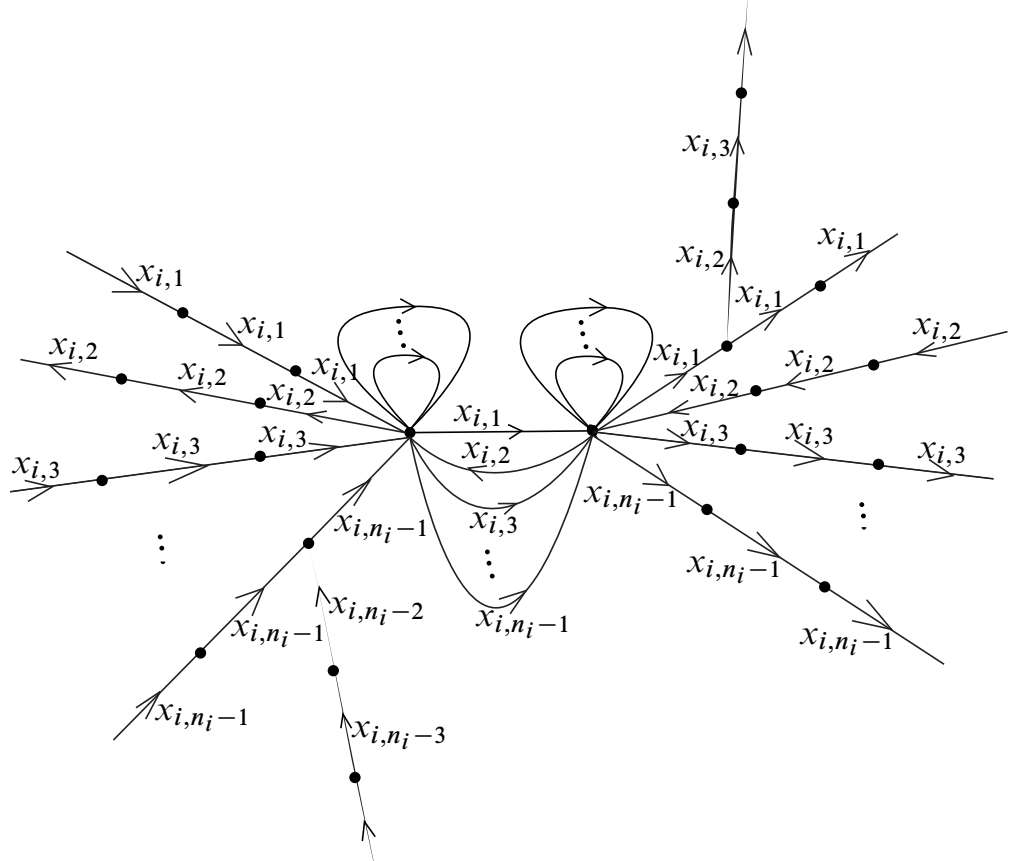

Figure 18

The resulting graph is not folded, but becomes folded graph after the following obvious folding operation around each inserted $\Omega_{k}$ :

- fold the subpath $x_{i, n_{i}-1} a_{i, 1} b_{i, 1} a_{i, 1}^{-1} b_{i, 1}^{-1} \cdots a_{i, g_{i}} b_{i, g_{i}} a_{i, g_{i}}^{-1} b_{i, g_{i}}^{-1}$ whose terminal vertex is the vertex $u_{i, n_{i}, k}^{+}$with the loops of $\Omega_{k}$ at the left vertex of $\Omega_{k}$ and then with the $x_{i, n_{i}-1}$-edge of $\mathcal{G}_{i, 4}(n)$ whose terminal vertex is the left vertex of $\Omega_{k}$, and

- fold the two $x_{1}$-edges whose initial vertices are the right vertex of $\Omega_{k}$.

The resulting folded graph $\mathcal{G}_{i, 4}(n)$ around the inserted $\Omega_{k}$ is shown in Figure 18. By our construction we see that $\mathcal{G}_{i, 4}(n)$ is a folded, $L_{i}$-labeled, directed graph, with no $x_{i, j}$-loops, with each of the words $w_{i, 1}^{*}, \ldots, w_{i, \ell_{i}}^{*}$ still representable by a loop based at $v_{i, 0}$, and with each of the words $y_{i, 1}, \ldots, y_{i, r_{i}}$ still representable by a non-closed path based at $v_{i, 0}$. Also we see that the graph $\mathcal{G}_{i, 4}(n)$ contains loops based $v_{i, 0}$ representing the words $z_{i, j, k}(n+1)^{*}$, for any $j=1, \ldots, n_{i}, k=1, \ldots, d_{i}-1$.

The graph $\mathcal{G}_{i, 4}(n)$ is not $L_{i}$-regular yet since it does not contain any $x_{i, j}$-loops. So it must contain a missing label. Let $x \in L_{i}$ be a missing label at a vertex $v$ of $\mathcal{G}_{i, 4}(n)$. Let $\alpha$ be a finite directed graph consisting of a single path of edges all labeled with $x$, as shown in Figure 19. We identify the left end vertex of $\alpha$ to the vertex $v$ of $\mathcal{G}_{i, 4}(n)$. The resulting graph $\mathcal{G}_{i, 5}(n)$ is obviously still folded, contains $\mathcal{G}_{i, 4}(n)$ as an embedded subgraph, and contains no $x_{i, j}$-loops for any $j=1, \ldots, n_{i}$. By choosing a 
long enough path $\alpha$, we may assume that the number of vertices of $\mathcal{G}_{i, 5}(n)$ is bigger than $d_{i}(n+1)+1$.

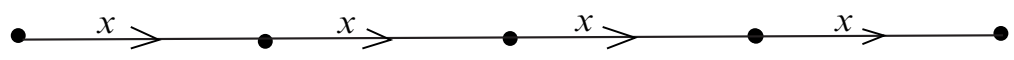

Figure 19

Now by Remark 9.7, we can obtain an $L_{i}$-regular graph $\mathcal{G}_{i, 6}(n)$ such that

(1) $\mathcal{G}_{i, 5}(n)$ is an embedded subgraph of $\mathcal{G}_{i, 6}(n)$; thus in particular in $\mathcal{G}_{i, 6}(n)$ each of the words $w_{i, 1}^{*}, \ldots, w_{i, \ell_{i}}^{*}, z_{i, j, k}(n+1)^{*}, j=1, \ldots, n_{i}, k=1, \ldots, d_{i}-1$ is representable by a loop based at $v_{i, 0}$, and each of the words $y_{i, 1}, \ldots, y_{i, r_{i}}$ is representable by a non-closed path based at $v_{i, 0}$;

(2) $\mathcal{G}_{i, 6}(n)$ contains no loops representing the word $x_{i, j}^{l}$ for any $j=1, \ldots, n_{i}$, $l=1, \ldots, m_{i}^{*}-1$, where $m_{i}^{*}$ is the number of vertices of $\mathcal{G}_{i, 6}(n)$.

Note that $m_{i}^{*}$ is some integer larger than $d_{i}(n+1)+1$. Let $N_{i}=m_{i}^{*}-\left(d_{i}-1\right)(n+1)-1$. Then $N_{i}>(n+1)$.

During the transformation from $\mathcal{G}_{i, 4}(n)$ to $\mathcal{G}_{i, 6}(n)$, the subgraph of $\mathcal{G}_{i, 4}(n)$ consisting of the edges which intersect the subgraph $\Omega_{k}$ (for each fixed $k=1, \ldots, d_{i}-1$ ) remained unchanged since $\mathcal{G}_{i, 4}(n)$ was locally $L_{i}$-regular already at the two vertices of $\Omega_{k}$. Now we replace $\Omega_{k}$, for each of $k=1, \ldots d_{i}-1$, by a graph similar to $\Omega$ but with $N_{i}-n+1 \geq 3$ vertices (Figure 20 illustrates such a graph with four vertices). Then the resulting graph $\mathcal{G}_{i, 7}(n)$ has the following properties.

(1) $\mathcal{G}_{i, 7}(n)$ is $L_{i}$-regular;

(2) each of the words $y_{i, 1}, \ldots, y_{i, r_{i}}$ is still representable by a non-closed path based at $v_{i, 0}$ in $\mathcal{G}_{i, 7}(n)$;

(3) each of the words $w_{i, 1}^{*}, \ldots, w_{i, \ell_{i}}^{*}$ is still representable by a loop based at $v_{i, 0}$ in $\mathcal{G}_{i, 7}(n)$;

(4) $\mathcal{G}_{i, 7}(n)$ contains no loops representing the word $x_{i, j}^{l}$ for each $j=1, \ldots, n_{i}$ and each $l=1, \ldots, m_{i}-1$, where $m_{i}$ is the number of vertices of $\mathcal{G}_{i, 7}$;

(5) $\mathcal{G}_{i, 7}(n)$ contains a closed loop based at $v_{i, 0}$ representing the word $z_{i, j, k}\left(N_{i}\right)^{*}$, for each $j=1, \ldots, n_{i} ; k=1, \ldots, d_{i}-1$; and

(6) $m_{i}$, the number of vertices of $\mathcal{G}_{i, 7}(n)$, is equal to $N_{i} d_{i}+1$. 
Properties (1)-(5) are obvious by the construction, while property (6) follows by a simple calculation. Indeed

$$
\begin{aligned}
m_{i} & =m_{i}^{*}+\left(N_{i}-n+1-2\right)\left(d_{i}-1\right) \\
& =\left[N_{i}+\left(d_{i}-1\right)(n+1)+1\right]+\left(N_{i}-(n+1)\right)\left(d_{i}-1\right) \\
& =N_{i} d_{i}+1 .
\end{aligned}
$$

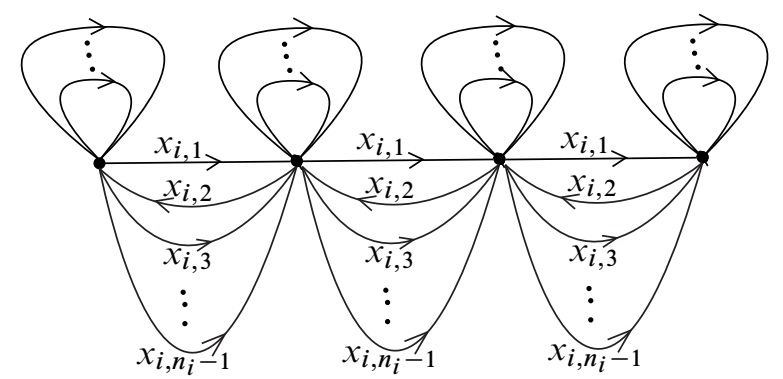

Figure 20

Case (b) $n_{i}>1$ is odd

We modify the graph $\mathcal{G}_{i, 0}(n)^{f}$ as follows. For each of $k=1, \ldots, d_{i}-1$, we define a subset of vertices $\mathcal{U}_{i, k}=\left\{u_{i, j, k}: 1 \leq j \leq n_{i}\right\} \cup\left\{u_{i, n_{i}, k}^{\prime}\right\} \subset \mathcal{G}_{i, 0}(n)^{f}$, where

- if $j \leq n_{i}-1$, then $u_{i, j, k}$ is a vertex in $\Phi_{i, j, k}(n)$, such that there are at least three edges after it and at least three edges before it in the directed path $\Phi_{i, j, k}(n)$;

- $u_{i, n_{i}, k}$ is the initial vertex of an edge labeled $x_{1}$ in $\Phi_{i, n_{i}, k}(n)$ such that there are at least three edges with label $x_{1}$ before it in the directed path $\Phi_{i, j, k}(n)$; and $-u_{i, n_{i}, k}^{\prime}$ is the initial vertex of an edge with label $x_{2}$ in $\Phi_{i, j, k}(n)$ which appears after the vertex $u_{i, j, k}$ in the directed path $\Phi_{i, j, k}$. We also insist that $\Phi_{i, j, k}(n)$ contains at least three edges with label $x_{1}$ between $u_{i, n_{i}, k}$ and $u_{i, n_{i}, k}^{\prime}$ and at least three edges with label $x_{1}$ after $u_{i, n_{i}, k}^{\prime}$.

Then cut $\mathcal{G}_{i, 0}(n)^{f}$ at the vertices of $\mathcal{U}_{i, k}, k=1, \ldots, d_{i}-1$, and for each $k$, insert the graph $\Omega_{k}$, which is a copy of the graph $\Omega$ shown in Figure 17 (b). That is, we do the following:

(1) Form a cut graph $\mathcal{G}_{i, 0}(n)_{c}^{f}=\mathcal{G}_{i, 0}(n)^{f} \backslash\left\{\mathcal{U}_{i, k} ; k=1, \ldots, d_{i}-1\right\}$, defined as in Case (a), with obvious modifications, ie we have similarly defined pairs of vertices $u_{i, j, k}^{ \pm}, u_{i, n_{i}, k}^{\prime \pm}$ for $\mathcal{G}_{i, 0}(n)_{c}^{f}$ such that if each such \pm pair of vertices are identified, then the resulting graph is the original $\mathcal{G}_{i, 0}(n)^{f}$.

(2) For each fixed $k=1, \ldots, d_{i}-1$, we identify the vertex set $\left\{u_{i, j, k}^{ \pm}, u_{i, n_{i}, k}^{\prime \pm}, j=\right.$ $\left.1, \ldots, n_{i}\right\}$ of $\mathcal{G}_{i, 0}(n)_{c}^{f}$ with the left and right-most vertices of $\Omega_{k}$ as follows: 
- if $j<n_{i}$, and $j=1$ or $j$ is even, then identify $u_{i, j, k}^{+}$with the left-most vertex of $\Omega_{k}$ and $u_{i, j, k}^{-}$with the right-most vertex;

- if $j<n_{i}, j \neq 1$ and $j$ is odd, then identify $u_{i, j, k}^{+}$with the right-most vertex of $\Omega_{k}$ and $u_{i, j, k}^{-}$with the left-most vertex;

- identify $u_{i, n_{i}, k}^{+}$with the left-most vertex of $\Omega_{k}$ and identify $u_{i, n_{i}, k}^{-}$with the right-most vertex of $\Omega_{k}$;

- identify $u_{i, n_{i}, k}^{\prime+}$ with the left-most vertex of $\Omega_{k}$ and identify $u_{i, n_{i}, k}^{\prime}$ with the right-most vertex of $\Omega_{k}$.

The resulting graph is not folded, but becomes folded graph after the following folding operations are performed around each inserted $\Omega_{k}$ :

- fold the path $x_{i, n_{i}-1} a_{i, 1} b_{i, 1} a_{i, 1}^{-1} b_{i, 1}^{-1} \cdots a_{i, g_{i}} b_{i, g_{i}} a_{i, g_{i}}^{-1} b_{i, g_{i}}^{-1}$ whose terminal vertex is the vertex $u_{i, n_{i}, k}^{+}$with the loops of $\Omega_{k}$ at the left-most vertex of $\Omega_{k}$ and then with the $x_{i, n_{i}-1}$-edge of $\mathcal{G}_{i, 4}(n)$ whose terminal vertex is the left-most vertex of $\Omega_{k}$;

- fold the two $x_{i, 1}$-edges whose initial vertices are the right-most vertex of $\Omega_{k}$;

- fold the two $x_{i, 1}$-edges whose terminal vertices are the left-most vertex of $\Omega_{k}$;

- fold the two $x_{i, 2}$-edges whose initial vertices are the right-most vertex of $\Omega_{k}$.

The resulting folded graph $\mathcal{G}_{i, 4}(n)_{0}^{f}$ around the inserted $\Omega_{k}$ is shown in Figure 21. By our construction we see that $\mathcal{G}_{i, 4}(n)^{f}$ is a folded, $L_{i}$-labeled, directed graph, with no $x_{i, j}$-loops, with each of the words $w_{i, 1}^{*}, \ldots, w_{i, \ell_{i}}^{*}$ still representable by a loop based at $v_{i, 0}$, and with each of the words $y_{i, 1}, \ldots, y_{i, r_{i}}$ still representable by a non-closed path based at $v_{i, 0}$. Also we see that the graph $\mathcal{G}_{i, 4}(n)$ contains loops based $v_{i, 0}$ representing the words $z_{i, j, k}(n+2)^{*}$, for all $j=1, \ldots, n_{i}, k=1, \ldots, d_{i}-1$.

We then define $\mathcal{G}_{i, 5}(n)$ and $\mathcal{G}_{i, 6}(n)$ in a similar manner as Case (a); here we may assume that $\mathcal{G}_{i, 5}(n)$ has at least $\left(d_{i}-1\right)(n+2)-1$ vertices. Let $m_{i}^{*}$ be the number of vertices of $\mathcal{G}_{i, 6}$, and let $N_{i}=m_{i}^{*}-\left(d_{i}-1\right)(n+2)-1$. To form $\mathcal{G}_{i, 7}(n)$, we replace each graph $\Omega_{k}, k=1, \ldots, d_{i}-1$ in $\mathcal{G}_{i, 6}(n)$ with a graph similar to Figure 17(b) but with $1+N_{i}-n$ vertices. In the current case, we need $1+N_{i}-n$ to be an odd integer in order for the construction to work. (Figure 22 illustrates such a graph with five vertices). This is made possible by the following lemma.

Lemma 11.3 $N_{i}-n$ is even. 


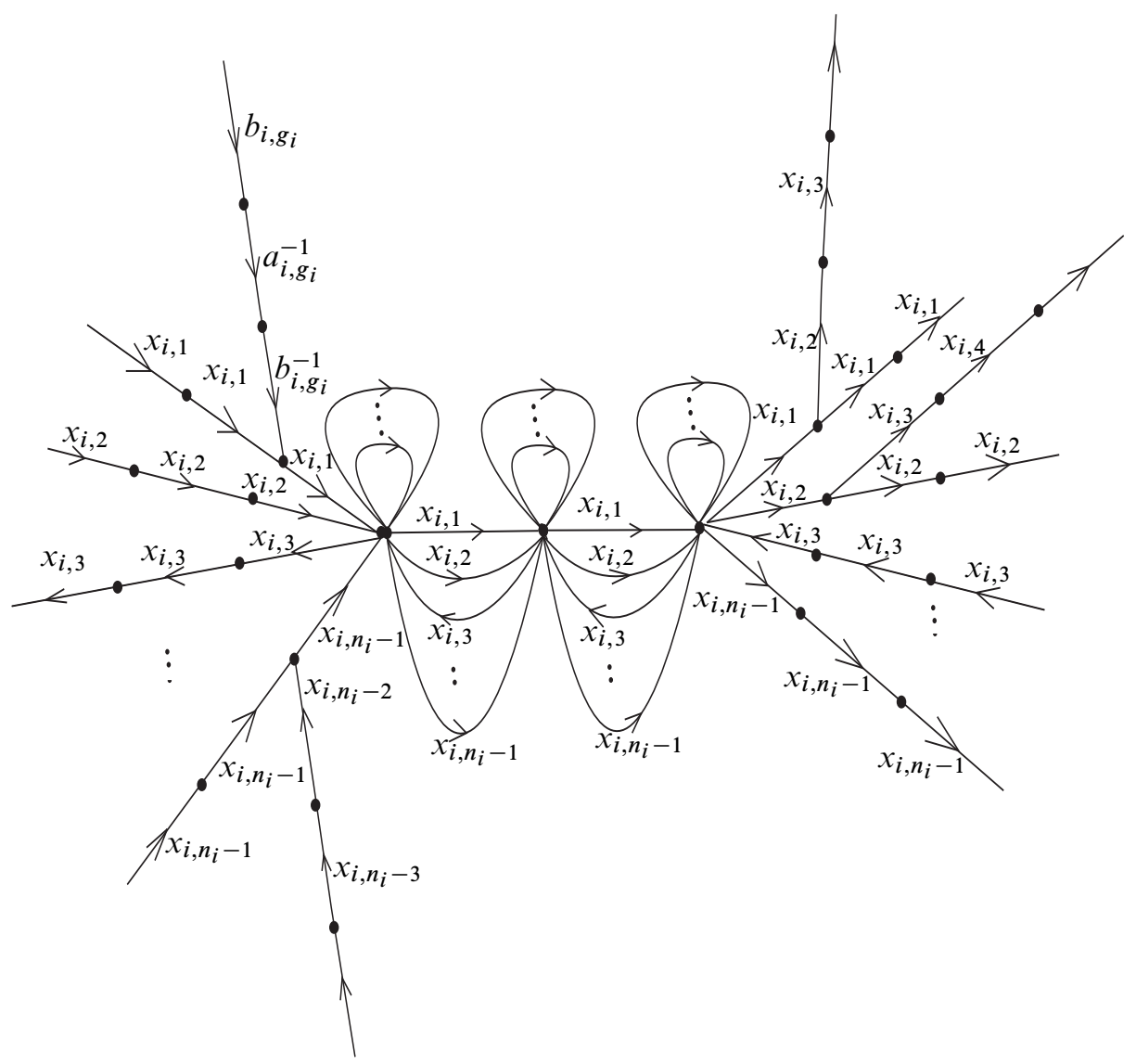

Figure 21

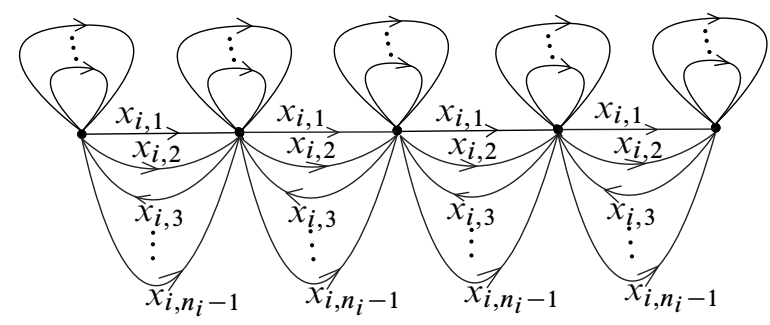

Figure 22

Proof Since $n_{i}$ is odd, then the Euler characteristic $\chi\left(S_{i}^{-}\right)$of $S_{i}^{-}$is odd. Let $\widehat{S}_{i}^{-}$ be the cover of $S_{i}^{-}$corresponding to the subgroup $L\left(\mathcal{G}_{i, 6}(n), v_{i, 0}\right)$ of $\Gamma_{i}$. Due to the property (2) of the graph $\mathcal{G}_{i, 6}, \widehat{S}_{i}^{-}$also has $n_{i}$ boundary components (cf the second paragraph in the proof of Proposition 11.1). So $\chi\left(\widehat{S}_{i}^{-}\right)$is also odd. Therefore the degree 
of the cover, which is $m_{i}^{*}$, must be odd. We have that $m_{i}^{*}=N_{i}+\left(d_{i}-1\right)(n+2)+1$. Since $n_{i}$ is odd, $n_{i_{*}}$ is even (see Section 5). Thus $d_{i}-1=\Delta n_{i_{*}}-1$ is odd. Thus $N_{i}$ and $n$ are both even or both odd. So $N_{i}-n$ is even.

The rest of the argument proceeds by obvious analogy with the case where $n_{i}$ is even. That is, the graph $\mathcal{G}_{i, 7}(n)$ is a graph with the properties listed as (1)-(6) in Case (a). Indeed, Properties (1)-(5) are immediate. To verify Property (6), we let $m_{i}$ be the number of vertices of $\mathcal{G}_{i, 7}$, and then we have:

$$
\begin{aligned}
m_{i} & =m_{i}^{*}+\left(1+N_{i}-n-3\right)\left(d_{i}-1\right) \\
& =N_{i}+\left(d_{i}-1\right)(n+2)+1+\left(N_{i}-n-2\right)\left(d_{i}-1\right) \\
& =N_{i} d_{i}+1
\end{aligned}
$$

Case (c) $n_{i}=1$

We modify the graph $\mathcal{G}_{i, 0}(n)^{f}$ as follows. For each of $k=1, \ldots, d_{i}-1$, we pick a pair vertices $\left\{u_{i, k}, u_{i, k}^{\prime}\right\}$ in $\Phi_{i, 1, k}$ as follows:

$-u_{i, k}$ is the terminal vertex of an edge with label $a_{i, 1}$ in $\Phi_{i, j, k}(n)$ such that there are at least three edges with label $a_{i, 1}$ before $u_{i, k}$ in the directed path $\Phi_{i, j, k}(n)$; and

$-u_{i, k}^{\prime}$ is the terminal vertex of an edge with label $b_{i, 1}$ which appears after the vertex $u_{i, k}$. We also insist that there are at least three edges with label $b_{i, 1}$ between $u_{i, k}$ and $u_{i, k}^{\prime}$ and that there are at least three edges with label $b_{i, 1}$ after $u_{i, k}^{\prime}$ in the path $\Phi_{i, j, k}(n)$.

Then cut the graph $\mathcal{G}_{i, 0}(n)^{f}$ at all the pairs of vertices $\left\{u_{i, k}, u_{i, k}^{\prime}\right\}, k=1, \ldots, d_{i}-1$, and for each $k$, insert the graph $\Omega_{k}$-which is a copy of the graph $\Omega$ shown in Figure 17 (c)- as follows. Form a cut graph $\mathcal{G}_{i, 0}(n)_{c}^{f}=\mathcal{G}_{i, 0}(n)^{f} \backslash\left\{u_{i, k}, u_{i, k}^{\prime} ; k=1, \ldots, d_{i}-1\right\}$, and let $u_{i, k}^{ \pm}, u_{i, k}^{\prime \pm}$ be the corresponding vertices for $\mathcal{G}_{i, 0}(n)_{c}^{f}$. For each fixed $k=$ $1, \ldots, d_{i}-1$, we identify the vertex $u_{i, k}^{+}$with the left-most vertex of $\Omega_{k}$, identify $u_{i, k}^{-}$ with the right-most vertex of $\Omega_{k}$, identify $u_{i, k}^{\prime}$ with the right-most vertex of $\Omega_{k}$ and identify $u_{i, k}^{\prime}$ with the left-most vertex of $\Omega_{k}$.

The resulting graph is not folded, but becomes folded graph after a single folding operation around each inserted $\Omega_{k}$ : fold the two $a_{i, 1}$-edges whose terminal vertices are the right-most vertex of $\Omega_{k}$. The resulting folded graph $\mathcal{G}_{i, 4}(n)_{0}^{f}$ around the inserted $\Omega_{k}$ is shown in Figure 23. By our construction we see that $\mathcal{G}_{i, 4}(n)^{f}$ is a folded $L_{i}$-labeled directed graph, with no $x_{i, 1}$-loops, with each of the words $w_{i, 1}^{*}, \ldots, w_{i, \ell_{i}}^{*}$ still representable by a loop based at $v_{i, 0}$, and with each of the words $y_{i, 1}, \ldots, y_{i, r_{i}}$ still representable by a non-closed path based at $v_{i, 0}$. Also we see that 
the graph $\mathcal{G}_{i, 4}(n)$ contains loops based at $v_{i, 0}$ representing the words $z_{i, 1, k}(n+4)^{*}$, for all $k=1, \ldots, d_{i}-1$.

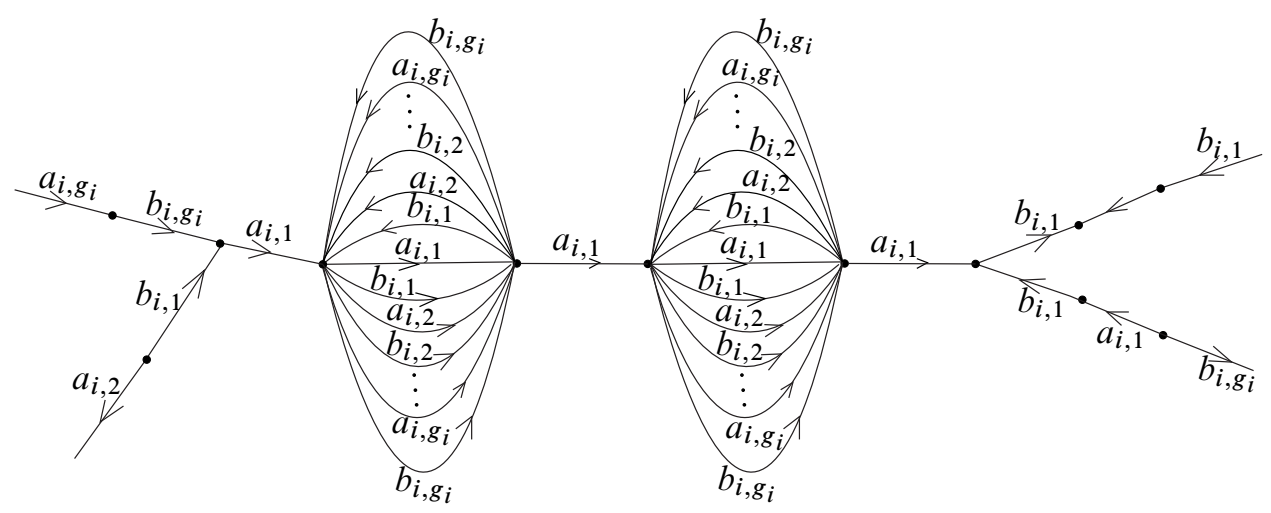

Figure 23

As in the previous case, we get $\mathcal{G}_{i, 5}(n)$ and $\mathcal{G}_{i, 6}(n)$. In the current case, $N_{i}=$ $m_{i}^{*}-\left(d_{i}-1\right)(n+4)-1$, which is assumed larger than $n+4$ (since $m_{i}^{*}$ can be assumed arbitrary large). To form $\mathcal{G}_{i, 7}(n)$, we replace the left half (with three vertices) of $\Omega_{k}$, for each $k=1, \ldots, d_{i}-1$, with a graph similar to Figure 17(c) but with $N_{i}-n-1$ vertices. In the current case, we also need $1+N_{i}-n$ to be an odd integer in order for the construction to work. This is true, and can be proved as in Case (b). It is easy to see that $\mathcal{G}_{i, 7}(n)$ has all the Properties (1)-(5). To verify Property (6), we have:

$$
\begin{aligned}
m_{i} & =m_{i}^{*}+\left(N_{i}-n-1-3\right)\left(d_{i}-1\right) \\
& =N_{i}+\left(d_{i}-1\right)(n+4)+1+\left(N_{i}-n-4\right)\left(d_{i}-1\right) \\
& =N_{i} d_{i}+1
\end{aligned}
$$

\section{HS-manifolds}

We call a compact, connected, orientable 3 -manifold $W$ with boundary is an HSmanifold if it has the form $W=H \cup(S \times I)$, where:

(i) each component of $H$ is a handlebody of genus at least one;

(ii) each component of $S$ is a compact orientable surface with boundary;

(iii) $H \cap(S \times I)=\partial S \times I$;

(iv) each component of $H \cap(S \times I)=\partial S \times I$ is an annulus in $\partial H$ which is homotopically non-trivial in $H$. 
Lemma 12.1 Let $W=H \cup(S \times I)$ be an HS-manifold. Let $A$ denote the set of annuli $H \cap(S \times I)=\partial S \times I$. Suppose that $S$ has no disk components, and that for every compressing disk $D$ of $H$, the set $D \cap A$ has at least two components. Then $W$ has incompressible boundary.

Proof Suppose otherwise that $\partial W$ is compressible in $W$. Let $(B, \partial B) \subset(W, \partial W)$ be a compressing disk. Isotope $B$ so that it intersects the set of annuli $A$ in a collection of properly embedded arcs and simple closed curves. Since no component of $S$ is a disk, and since each component of $A$ is non-trivial in $H$, we can remove, by isotopy of $B$, all simple closed curve components of $B \cap A$ (by a standard inner-most argument, using also the fact that $H$ and $S \times I$ are irreducible 3-manifolds).

Note that the intersection $A \cap B$ cannot be empty since otherwise $B$ would be contained in $S \times I-A$ but each component of $\partial(S \times I)-A$ is incompressible in $S \times I$.

We may also assume that each arc component of $A \cap B$ is essential in $A$. For otherwise we can surger the disk $B$ along an outer-most such arc in $A$ to get a compressing disk of $W$ whose intersection with $A$ has fewer components.

Now $B \cap A$ is a set of arcs, each of which is essential in $A$. Let $\alpha$ be a component of $B \cap A$ which is outer-most in $B$. Let $\beta$ be the component of $\partial B \backslash \partial \alpha$ whose interior is disjoint from $A$, and let $B_{1}$ be the sub-disk of $B$ co-bounded by $\alpha$ and $\beta$. Then $B_{1} \cap A=\partial B_{1} \cap A=\alpha$, and thus if $B_{1}$ is contained in $H$, then it must be an essential compressing disk in $H$. But by our assumption no such compressing disk exists. On the other hand, there is no properly embedded disk in $S \times I$ which intersects $\partial S \times I$ in a single essential arc.

\section{Proof of Theorem 1.1}

In Section 11, we found, for each $i=1$, 2, a finite cover $\breve{Y}_{i}=\breve{S}_{i} \times I$ of $Y_{i}=S_{i} \times I$, such that the map $g_{i}: J_{i}^{-} \rightarrow Y_{i}^{-}$lifts to an embedding $\breve{g}_{i}: J_{i}^{-} \rightarrow \breve{Y}_{i}^{-}$, and the $d$ components of $\breve{g}_{i}\left(\partial_{p} J_{i}^{-}\right)$are evenly spaced in $\partial_{p} \breve{Y}_{i}^{-}$, far apart from each other in $\partial_{p} \breve{Y}_{i}^{-}$. Recall from Section 6 that $K_{i}$ is an embedded submanifold of $J_{i}$ with an $R$-collared neighborhood in $J_{i}$, and that $\left(K_{i}^{-}, \partial_{p} K_{i}^{-}\right)$is properly embedded in the pair $\left(J_{i}^{-}, \partial_{p} J_{i}^{-}\right)$, with a relative $R$-collared neighborhood. It follows that the pair $\left(\breve{g}_{i}\left(K_{i}^{-}\right), \breve{g}_{i}\left(\partial_{p} K_{i}^{-}\right)\right)$has a relative $R$-collared neighborhood in $\left(\breve{Y}_{i}^{-}, \partial_{p} \breve{Y}_{i}^{-}\right)$.

Also recall from Section 6 that $K_{1}^{-}$and $K_{2}^{-}$are isometric under the isometry $h: K_{1} \rightarrow$ $K_{2}$. Thus there is a corresponding isometry from $\breve{g}_{1}\left(K_{1}^{-}\right)$to $\breve{g}_{2}\left(K_{2}^{-}\right)$, which is $\breve{g}_{2} \circ h \circ \breve{g}_{1}^{-1}$. 
Now let $\breve{Y}^{-}$be the union of $\breve{Y}_{1}^{-}$and $\breve{Y}_{2}^{-}$with $\breve{g}_{1}\left(K_{1}^{-}\right)$and $\breve{g}_{2}\left(K_{2}^{-}\right)$identified by the isometry. Let $U_{k}^{-} \subset \breve{Y}^{-}$be the identification of $\breve{g}_{1}\left(K_{1, k}^{-}\right)$with $\breve{g}_{2}\left(K_{2, k}^{-}\right), k=1, \ldots, q$, and let $U^{-}$be the disjoint union of $U_{k}^{-}$'s. Then $\breve{Y}^{-}$is a connected metric space, with a path metric induced from the metrics on $Y_{1}^{-}$and $Y_{2}^{-}$. There is an induced local isometry $f: \breve{Y}^{-} \rightarrow M$.

Define the parabolic boundary, $\partial_{p} \breve{Y}^{-}$, of $\breve{Y}^{-}$to be the union of $\partial_{p} \breve{Y}_{1}^{-}$and $\partial_{p} \breve{Y}_{2}^{-}$, with $\breve{g}_{1}\left(\partial_{p} K_{1}\right)$ and $\breve{g}_{2}\left(\partial_{p} K_{2}\right)$ identified by the isometry $\breve{g}_{2} \circ h \circ \breve{g}_{1}^{-1}$. The parabolic boundary of $U^{-}$is defined to be the identification of $\breve{g}_{1}\left(\partial_{p} K_{1}\right)$ and $\breve{g}_{2}\left(\partial_{p} K_{2}\right)$. Let $D_{j}, j=1, \ldots, d$, be the components of the parabolic boundary $\partial_{p} U^{-}$of $U^{-}$, and let $s_{j}$ be the topological center point of $D_{j}$ (ie the $s_{j}$ 's are the intersection points of $\partial \breve{S}_{1}^{-}$ and $\partial \breve{S}_{2}^{-}$in $\left.\partial_{p} \breve{Y}^{-}\right)$. Since $\breve{g}_{i}\left(K_{i}^{-}\right)$has an $R$-collared neighborhood in $\breve{Y}_{i}^{-}$, then $U^{-}$ has an $R$-collared neighborhood in $\breve{Y}^{-}$.

Recall $f_{i}:\left(S_{i}^{-}, \partial S_{i}^{-}\right) \rightarrow\left(M^{-}, \partial M^{-}\right)$is a proper map, such that $\left.f_{i}\right|_{\partial S_{i}^{-}}: \partial S_{i}^{-} \rightarrow \partial M$ is an embedding for each $i=1,2$. Let $\beta_{i, j}^{*}=f_{i}\left(\beta_{i, j}\right)$. Then $\Delta$ is the intersection number between $\beta_{1,1}^{*}$ and $\beta_{2,1}^{*}$, and $t_{1}, \ldots, t_{d}$ are the $d=n_{1} n_{2} \Delta$ intersection points between $\left\{\beta_{1, j}^{*}, j=1, \ldots, n_{1}\right\}$ and $\left\{\beta_{2, j}^{*}, j=1, \ldots, n_{2}\right\}$ (since each $\beta_{i, j}^{*}$ is a Euclidean circle in the Euclidean torus $\partial M^{-}$). Recall also that $\breve{\beta}_{i, j}, j=1, \ldots, n_{i}$, are boundary components of $\partial \breve{S}_{i}^{-}$, and each $\breve{\beta}_{i, j}$ is the cyclic covering of $\beta_{i, j}$ of order $m_{i}=N_{i} d_{i}+1$. Recall that by our convention, $t_{1}$ is the base point for each of $M, M^{-}$, $C$ and $T=\partial M=\partial C$, and that $t_{1}$ is one of intersection points between $\beta_{1,1}^{*}$ and $\beta_{2,1}^{*}$. We may consider $\beta_{1,1}^{*}$ and $\beta_{2,1}^{*}$ as two elements in $\pi_{1}\left(T, t_{1}\right)=\pi_{1}\left(C, t_{1}\right)$. Now let $\mathcal{A}$ be the subgroup of $\pi_{1}\left(T, t_{1}\right)$ generated by the two elements $\left(\beta_{1,1}^{*}\right)^{m_{1}}$ and $\left(\beta_{2,1}^{*}\right)^{m_{2}}$. Then $\mathcal{A}$ is a rank two subgroup of $\pi_{1}\left(T, t_{1}\right)=\pi_{1}\left(C, t_{1}\right)$ of finite index. Let $p_{0}: \breve{C} \rightarrow C$ be the covering corresponding to $\mathcal{A}$. By our construction, $\partial_{p} \breve{Y}^{-}$can be embedded isometrically in $\breve{T}=\partial \breve{C}$ such that $p_{0}: \breve{\beta}_{i, j} \rightarrow \beta_{i, j}^{*}$ is the map $\breve{\beta}_{i, j} \rightarrow \beta_{i, j} \rightarrow \beta_{i, j}^{*}$ for each $j=1, \ldots, n_{i}$. Thus the geometric intersection number in $\breve{T}$ between $\breve{\beta}_{1,1}$ and $\breve{\beta}_{2,1}$ is equal to $\Delta$ and there are $d=n_{1} n_{2} \Delta$ intersection points $\left\{s_{k}, k=1, \ldots, d\right\}$ between $\left\{\breve{\beta}_{1, j}, j=1, \ldots, n_{1}\right\}$ and $\left\{\breve{\beta}_{2, j}, j=1, \ldots, n_{2}\right\}$. We may assume that the $s_{k}$ 's are indexed so that $p_{0}\left(s_{k}\right)=t_{k}, k=1, \ldots, d$. From the construction of Section 11 , the points $\left\{s_{k}, k=1, \ldots, d\right\}$ divide the circles $\left\{\breve{\beta}_{i, j}, j=1, \ldots, n_{1}\right\}$ into segments, each of which has wrapping number $N_{i}$. Thus $\left\{\breve{\beta}_{i, j}, i=1,2, j=1, \ldots, n_{i}\right\}$ divides the torus $\breve{T}$ into a set of Euclidean parallelograms with long sides (because the wrapping numbers $N_{1}$ and $N_{2}$ can be chosen arbitrarily large).

We now replace the $R$-collared neighborhood of $U^{-}$in $\breve{Y}^{-}$by a hyperbolic 3manifold $\bar{U}^{-}$, whose construction is given below, such that:

(i) $\bar{U}^{-}$is a thickening of $U^{-}$; 
(ii) the new space $Y^{-}=\breve{Y}_{1}^{-} \cup \bar{U}^{-} \cup \breve{Y}_{2}^{-}$is a connected, compact, hyperbolic 3manifold, locally convex everywhere except on its parabolic boundary, whose metric restricts to the original metric on $\breve{Y}^{-}$;

(iii) $Y^{-}$has a local isometry $f: Y^{-} \rightarrow M$ which extends the local isometries $f_{i} \circ \breve{p}_{i}: \breve{Y}_{i}^{-} \rightarrow M$;

(iv) the parabolic boundary of $Y^{-}$is a regular neighborhood of that of $\breve{Y}^{-}$in the torus $\breve{T}$ and thus the complement of $\partial_{p}\left(Y^{-}\right)$in $\breve{T}$ is a set of "round-cornered parallelograms" in $\breve{T}$ (cf Figure 25);

(v) since each such parallelogram given in (iv) has very long sides, we can cap off $\partial_{p} Y^{-}$with a solid cusp $C_{0}$; the resulting manifold $Y$ is a convex, hyperbolic 3 - manifold with a cusp and $Y$ has a local isometry into $M$.

We now provide more details. First we construct $\bar{U}^{-}$, component-wise. We illustrate the construction of $\bar{U}_{k}^{-}$for the component $U_{k}^{-}$of $U^{-}$. Recall the construction of $K_{i, k}$ given in Section 6. It is the quotient space of $Z_{i, j_{k}} \subset X_{i}$ under the group $\Gamma_{i, j_{k}}$. Recall that $\breve{q}_{i}: X_{i} \rightarrow \breve{Y}_{i}$ is the universal covering map. Thus $Z_{i, j_{k}}^{-}$is the universal cover of $\breve{g}_{i}\left(K_{i, k}^{-}\right)$under the map $\breve{q}_{i}$. Also there are elements $\gamma_{i, j_{k}} \in \Gamma$ such that $X_{i, j_{k}}=\gamma_{i, j_{k}}\left(X_{i}\right), W_{j_{k}}=X_{1, j_{k}} \cap X_{2, j_{k}}$, and $Z_{i, j_{k}}=\gamma_{i, j_{k}}^{-1}\left(W_{j_{k}}\right)$. The space $W_{j_{k}}$ is invariant under the action of the group $\gamma_{1, j_{k}} \Gamma_{1} \gamma_{1, j_{k}}^{-1} \cap \gamma_{2, j_{k}} \Gamma_{2} \gamma_{2, j_{k}}^{-1}$.

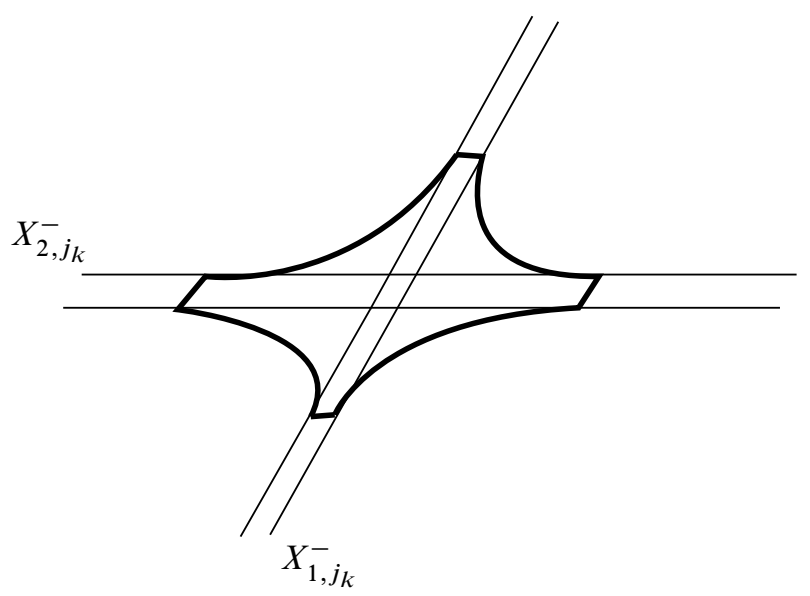

Figure 24: The plane region enclosed in the thickened curve is the component of the parabolic boundary of $\bar{W}_{j_{k}}^{-}$in $\partial B_{\infty}$.

Now let Hull $\left(X_{1, j_{k}} \cup X_{2, j_{k}}\right)$ be the convex hull of $X_{1, j_{k}} \cup X_{2, j_{k}}$ in $\mathbb{H}^{3}$, and let $N_{R}\left(W_{j_{k}}\right)$ be the $R$-collared neighborhood of $W_{j_{k}}$ in $\mathbb{H}^{3}$. Then $\operatorname{Hull}\left(X_{1, j_{k}} \cup X_{2, j_{k}}\right)-$ 
$\left(X_{1, j_{k}} \cup X_{2, j_{k}}\right) \subset N_{R}\left(W_{j_{k}}\right)$ by Proposition 4.5. Let $\bar{W}_{j_{k}}=N_{R}\left(W_{j_{k}}\right) \cap \operatorname{Hull}\left(X_{1, j_{k}} \cup\right.$ $X_{2, j_{k}}$ ), and $\bar{W}_{j_{k}}^{-}=\bar{W}_{j_{k}} \backslash \mathcal{B}$. We call $\bar{W}_{j_{k}}^{-} \cap \mathcal{B}$ the parabolic boundary of $\bar{W}_{j_{k}}$. Note that $\bar{W}_{j_{k}}^{-}$is invariant under the action of $\gamma_{1, j_{k}} \Gamma_{1} \gamma_{1, j_{k}}^{-1} \cap \gamma_{2, j_{k}} \Gamma_{2} \gamma_{2, j_{k}}^{-1}$. The component of the parabolic boundary of $\bar{W}_{j_{k}}^{-}$in $\partial B_{\infty}$ is as shown in Figure 24. Let $\bar{U}_{k}^{-}=\bar{W}_{j_{k}}^{-} /\left(\gamma_{1, j_{k}} \Gamma_{1} \gamma_{1, j_{k}}^{-1} \cap \gamma_{2, j_{k}} \Gamma_{2} \gamma_{2, j_{k}}^{-1}\right)$. We now replace the $R$-collared neighborhood of $U_{k}^{-}$in $\breve{Y}^{-}$by $\bar{U}_{k}^{-}$; that is, we glue $\breve{Y}^{-} \backslash N_{\left(R, \breve{Y}^{-}\right)}\left(U_{k}^{-}\right)$with $\bar{U}_{k}^{-}$ along the frontier of $N_{\left(R, \breve{Y}^{-}\right)}\left(U_{k}^{-}\right)$in $\breve{Y}^{-}$(which is a part of the boundary of $\bar{U}_{k}$ ), using the original gluing map $\breve{g}_{2} \circ h \circ \breve{g}_{1}^{-1}$. We do this operation for each component of $U^{-}$. Because $U^{-}$has an $R$-collared neighborhood in $\breve{Y}^{-}$, the components $\bar{U}_{k}^{-}$ do not interfere with each other. That is, if we let $Y^{-}$denote the resulting space, then $\bar{U}_{k}^{-}, k=1, \ldots, q$, are mutually disjoint from each other in $Y^{-}$. Let $\bar{U}^{-}$be the union of $\bar{U}_{k}^{-}, k=1, \ldots, q$.

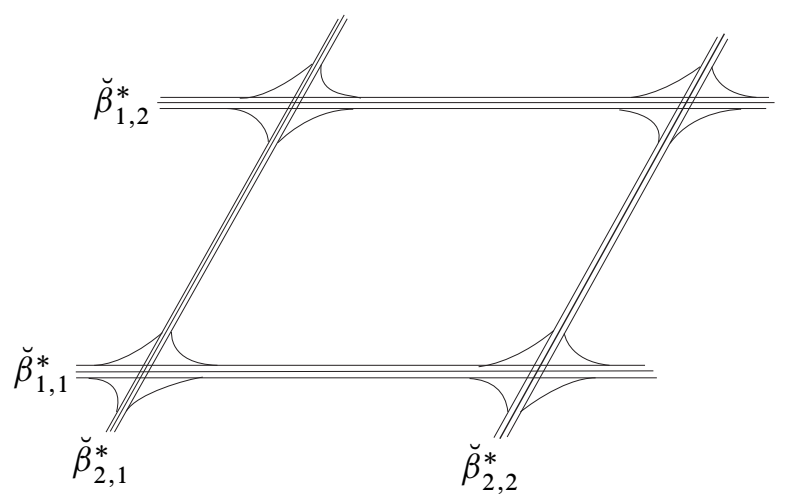

Figure 25: The parabolic boundary of $Y^{-}$

Lemma 13.1 $Y^{-}$is a connected, compact, hyperbolic 3-manifold containing $\breve{Y}_{i}^{-}$, $i=1,2$, as submanifolds (with their original hyperbolic structures), and there is a local isometry $f: Y^{-} \rightarrow M^{-}$extending the maps $f_{i} \circ \breve{p}_{i}: \breve{Y}_{i}^{-} \rightarrow M^{-}$.

Proof By Corollary 6.9, the frontier of $N_{\left(R, \breve{Y}_{i}^{-}\right)}\left(K_{i}^{-}\right)$in $\breve{Y}_{i}^{-}$is a set of (truncated) strips and annuli (the latter set may be empty) for each $i=1,2$. Note that the frontier of $N_{\left(R, \breve{Y}^{-}\right)}\left(U_{k}^{-}\right)$in $\breve{Y}^{-}$is the disjoint union of the frontier of $N_{\left(R, \breve{Y}_{i}^{-}\right)}\left(K_{i}^{-}\right)$in $\breve{Y}_{i}^{-}$, $i=1,2$. Hence $\breve{Y}^{-}$is obtained from gluing two 3 -manifolds along subsurfaces in their boundaries and thus is a manifold. Obviously it is a connected and compact 3-manifold. We just need to show that the hyperbolic structures of the gluing pieces match up over the identified region, forming a global hyperbolic structure on $Y^{-}$. 
It is enough to verify this around each component of $\bar{U}^{-}$. From the construction of $\bar{U}_{k}^{-}$ given above, we see that $X_{1, j_{k}}^{-} \cup \bar{W}_{j_{k}}^{-} \cup X_{2, j_{k}}^{-}$is a hyperbolic 3-submanifold of $\mathbb{H}^{3}$. Also $X_{i, j_{k}}^{-}$is a universal cover of $\breve{Y}_{i}^{-}, i=1,2$, and $\bar{W}_{j_{k}}^{-}$is a universal cover of $\bar{U}_{k}^{-}$, so there is a natural map from $X_{1, j_{k}}^{-} \cup \bar{W}_{j_{k}}^{-} \cup X_{2, j_{k}}^{-}$to the manifold $\breve{Y}_{1}^{-} \cup \bar{U}_{k}^{-} \cup \breve{Y}_{2}^{-}$. This provides the required hyperbolic structure around the component $U_{k}^{-}$.

Finally, the map $f$ can be constructed by piecing together the maps $f_{i} \circ \breve{p}_{i}$, and then extending to $Y^{-}$in the obvious way.

Lemma 13.2 Each component of $\bar{U}^{-}$is a handlebody.

Proof Each component $\bar{U}_{k}^{-}$of $\bar{U}^{-}$is homeomorphic to $K_{i, k}^{-}$, and thus is compact and irreducible. Since the fundamental group of $\bar{U}_{k}^{-}$is isomorphic to a subgroup of the free group $\Gamma_{i}$, then $\bar{U}_{k}^{-}$is a handlebody.

The parabolic boundary $\partial_{p} Y^{-}$of $Y^{-}$in $\breve{T}$ is the union of the parabolic boundary of $\breve{Y}_{i}^{-}, i=1,2$, and that of $\bar{U}^{-}$(see Figure 25).

Now we are going to construct the cusp $C_{0}$ mentioned in (v) above. The horosphere $\partial B_{\infty}$ is a universal cover of $\breve{T}$. Let $p_{*}: \partial B_{\infty} \rightarrow \breve{T}$ be the covering map. Along each component of $p_{*}^{-1}\left(\left\{\breve{\beta}_{i, j}, i=1,2, j=1, \ldots, n_{i}\right\}\right)$ we place an appropriate translation of $X_{i}$ by an element of $\Gamma$, and at each point of $p_{*}^{-1}\left(\left\{s_{1}, \ldots, s_{d}\right\}\right)$ we place an appropriate translation of a component of $\left\{\bar{W}_{j_{1}}, \ldots, \bar{W}_{j_{q}}\right\}$ by an element of $\Gamma$. Let $Q$ denote the union of these manifolds.

Let $B_{\infty}^{0}$ be the horoball based at $\infty$ which is smaller than $B_{\infty}$ by distance one, ie the horizontal plane $\partial B_{\infty}^{0}$ is above $\partial B_{\infty}$ by distance one. Let $V_{0}$ be the region between the two horizontal planes $\partial B_{\infty}^{0}$ and $\partial B_{\infty}$, and let $Q_{0}=Q \cap V_{0}$. Let $\widetilde{C}_{0}$ be the convex hull of $Q_{0}$ in $\mathbb{H}^{3}$. Then obviously $\widetilde{C}_{0}$ is contained in $B_{\infty}$.

Lemma 13.3 If $n$ (and thus $N_{i}>n$ ) is large enough, then $\widetilde{C}_{0} \cap V_{0}=Q_{0}$.

Proof Consider the frontier of $Q_{0}$ in $V_{0}$. It is a set of infinitely many annuli. Let $A_{1}$ be one of them. Then every point $x$ in $A_{1}$ is a point in the boundary of some translation of $X_{i}$ or in the boundary of some translation of $\left\{\bar{W}_{1}, \ldots, \bar{W}_{q}\right\}$. The tangent plane $P_{x}$ of that manifold at $x$ (a geodesic plane) is not a vertical plane and thus its intersection with the horizontal plane $\partial B_{\infty}^{0}$ is a Euclidean circle of finite diameter $d_{x}$. Modulo the action of $\beta_{1,1}^{*}$ and $\beta_{2,1}^{*}$, the set $\left\{d_{x}, x \in A_{1}\right\}$ has an upper bound independent of the integer $n$. Also modulo the action of the abelian group $\mathcal{A}=\left\langle\left(\beta_{1,1}^{*}\right)^{m_{1}},\left(\beta_{2,1}^{*}\right)^{m_{2}}\right\rangle$, there are only finitely many different annuli in $F r_{V_{0}}\left(Q_{0}\right)$. Hence the set $\left\{d_{x}, x \in F r_{V_{0}}\left(Q_{0}\right)\right\}$ 
has an upper bound independent of the integer $n$. Therefore if $n$ is sufficiently large, each $P_{x}, x \in F r_{V_{0}}\left(Q_{0}\right)$, will only intersect $Q_{0}$ at $x$. Thus in forming the convex hull of $Q_{0}$, all the new points added are above the plane $\partial B_{\infty}^{0}$. (cf Figure 26). The lemma is proved.

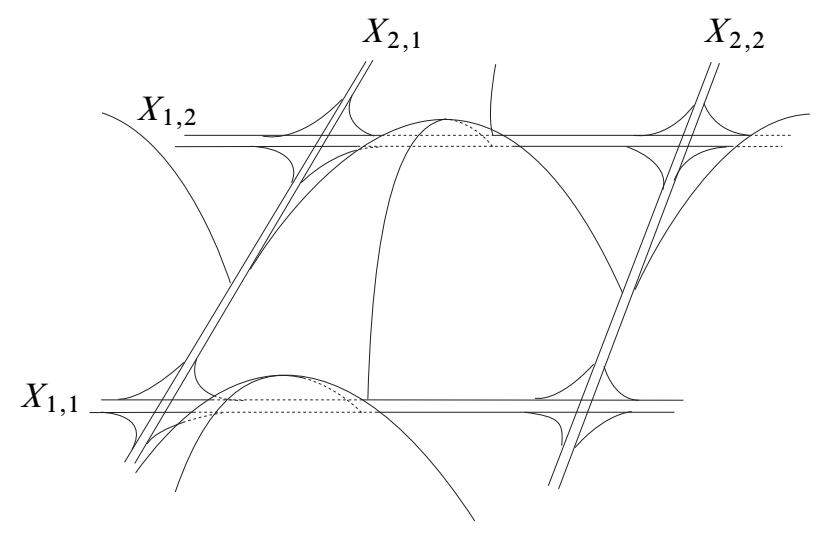

Figure 26: The convex hull above the plane $\partial B_{\infty}^{0}$

We may assume that $N_{i}>n$ has been chosen big enough so that the conclusion of Lemma 13.3 holds.

By our construction, $Q_{0}$ is invariant under the action of the abelian group $\mathcal{A}=$ $\left\langle\left(\beta_{1,1}^{*}\right)^{m_{1}},\left(\beta_{2,1}^{*}\right)^{m_{2}}\right\rangle$, and so is $\widetilde{C}_{0}$. Now let $C_{0}=\widetilde{C}_{0} / \mathcal{A}$. Then $C_{0}$ is contained in the cusp $\breve{C}$ and $C_{0} \cap \breve{T}=\partial_{p} Y^{-}$. Let $Y$ be the manifold which is the union of $Y^{-}$ and $C_{0}$ glued along the parabolic boundary of $Y^{-}$. We use the obvious gluing map, which is locally consistent with the gluing of $Q$ and $\widetilde{C}_{0}$ in $\mathbb{H}^{3}$. As in the proof of Lemma 13.1, one can show that $Y$ is a hyperbolic manifold with a local isometry $f$ into $M$. Moreover $Y$ is also convex. Indeed, we only need to check local convexity in a small neighborhood of $\partial_{p} Y^{-}$in $Y$, which holds, since the model space $Q \cup \widetilde{C}_{0}$ is locally convex in a small neighborhood of $\widetilde{C}_{0}$ in $Q \cup \widetilde{C}_{0}$.

Thus the local isometry $f$ induces an injection of $\pi_{1}\left(Y, s_{1}\right)$ into $\pi_{1}\left(M, t_{1}\right)$. We shall show:

Proposition 13.4 If $\Delta$ is bigger than one, or if both of $n_{1}$ and $n_{2}$ are bigger than one, then:

(1) the boundary of $Y$ is incompressible in $Y$;

(2) no essential loop in $\partial Y$ is freely homotopic into $C_{0}$. 
To prove Proposition 13.4, it is sufficient to show that every Dehn filling of $Y$ along its cusp $C_{0}$ gives a 3-manifold with incompressible boundary.

Let $Y(\alpha)$ be any Dehn filling of $Y$ along $C_{0}$ with slope $\alpha$. We claim that $Y(\alpha)$ is an HS-manifold (see Section 12). The handlebody part $H$ of $Y(\alpha)$ is $\bar{U}^{-} \cup C_{0}(\alpha)$, where $C_{0}(\alpha)$ is the filling of the cusp $C_{0}$ with slope $\alpha$. Indeed by Lemma 13.2 each component of $\bar{U}^{-}$is a handlebody which connects to the solid torus $C_{0}(\alpha)$ along its parabolic boundary $\partial_{p} \bar{U}^{-}$which is a set of disks. Thus $H=\bar{U}^{-} \cup C_{0}(\alpha)$ is a connected handlebody. The $S \times I$ part of $Y(\alpha)$ is $Y(\alpha) \backslash H=Y(\alpha) \backslash\left(\bar{U}^{-} \cup C_{0}(\alpha)\right)$. Indeed $Y(\alpha) \backslash$ $\left(\bar{U}^{-} \cup C_{0}(\alpha)\right)$ is the union of $\breve{Y}_{i}^{-} \backslash N_{\left(R, \breve{Y}_{i}^{-}\right)}\left(\breve{g}_{i}\left(K_{i}^{-}\right)\right)=\breve{Y}_{i}^{-} \backslash \breve{g}_{i}\left(\left(A N_{\left(R, X_{i}\right)}\left(K_{i}\right)\right)^{-}\right)$, $i=1,2$. It follows from Corollary 6.9 that $\breve{g}_{i}\left(\left(A N_{\left(R, X_{i}\right)}\left(K_{i}\right)\right)^{-}\right)$can be considered as $F_{i}^{-} \times I$ for some compact subsurface $F_{i}^{-}$of $\breve{S}_{i}^{-}$. Therefore each component of $\breve{Y}_{i}^{-} \backslash N_{\left(R, \breve{Y}_{i}^{-}\right)}\left(\breve{g}_{i}\left(K_{i}^{-}\right)\right)$can be given a trivial $I$-bundle structure over a compact surface with boundary such that the frontier in $\breve{Y}_{i}^{-}$consists of $I$-fibers (these $I$-fibers may not be consistent with the old $I$-fibers for $\breve{Y}_{i}^{-}$). The surface $S$ is compact, but is possibly disconnected.

Let $A=\partial S \times I$, which is the frontier of $\bigcup_{i=1,2} \breve{g}_{i}\left(\left(A N_{\left(R, X_{i}\right)}\left(K_{i}\right)\right)^{-}\right)$in $Y(\alpha)$ and is a set of mutually disjoint, properly embedded annuli in $Y(\alpha)$. By Lemma 12.1, we only need to show that for each compressing disk $D$ of $H, D \cap A$ has at least two components, and that each component of $S$ is not a disk. We deal with the latter requirement first.

Lemma 13.5 If $n$ (and thus $N_{i}>n$ ) is sufficiently large, then $S$ has no disk component.

Proof It is equivalent to show that if $n$ is sufficiently large then for each $i=1,2$, each component of $\breve{Y}_{i}^{-} \backslash \breve{g}_{i}\left(\left(A N_{\left(R, X_{i}\right)}\left(K_{i}\right)\right)^{-}\right)$is not simply connected.

Suppose otherwise that $\breve{Y}_{i}^{-} \backslash \breve{g}_{i}\left(\left(A N_{\left(R, X_{i}\right)}\left(K_{i}\right)\right)^{-}\right)$has a component $E_{0}$ which is simply connected (a 3-ball). We call the part of the boundary of $E_{0}$ which lies in $\partial_{p} \breve{Y}_{i}^{-}$the parabolic boundary of $E_{0}$ and denote it by $\partial_{p} E_{0}$. The union of the parabolic boundary and the frontier of $E_{0}$ in $\breve{Y}_{i}$ is an annulus $A_{0}$ in the boundary of $E_{0}$. The annulus $A_{0}$ can be decomposed by a set of parallel, essential arcs into components which are alternately components in $F_{\breve{Y}_{i}^{-}}\left(E_{0}\right)$ and $\partial_{p} E$. We call these components frontier faces and parabolic faces of $A_{0}$, respectively. Since the frontier of $\breve{g}_{i}\left(\left(A N_{\left(R, X_{i}\right)}\left(K_{i}\right)\right)^{-}\right)$in $\breve{Y}_{i}^{-}$has exactly $d$ components (Corollary 6.10), the annulus $A_{0}$ has at most $2 d$ faces. Note that every parabolic face of the annulus $A_{0}$ is a very long rectangle, depending on $n$, and that every frontier face of $A_{0}$ has a bounded diameter, independent of $n$. 
The 3-ball component $E_{0}$ has a lift, $\widetilde{E}_{0}$, to $X_{i}^{-}$, the universal cover of $\breve{Y}_{i}^{-}$. Note that $\widetilde{E}_{0}$ is isometric to $E_{0}$. Let $\widetilde{A}_{0}$ be an annulus in the boundary of $\widetilde{E}_{0}$ which is a lift of $A_{0}$. The annulus $\widetilde{A}_{0}$ has the corresponding decomposition into parabolic and frontier faces. Every parabolic face of $\tilde{A}_{0}$ is a long Euclidean rectangle contained in $\partial_{p} X_{i}^{-}=X_{i} \cap \partial \mathcal{B}_{i}$. Since $\Gamma$ acts transitively on components of $\mathcal{B}$, there is an element $\gamma$ of $\Gamma$ such that $\gamma\left(\tilde{A}_{0}\right)$ has a parabolic face $D_{0}$ which lies in $\partial B_{\infty}$.

Claim $\gamma\left(\tilde{A}_{0}\right)$ has only one parabolic face which lies in $\partial B_{\infty}$.

Since $\gamma\left(\widetilde{E}_{0}\right)$ is contained in $\gamma\left(X_{i}^{-}\right)$, we only need to show that $\gamma\left(\widetilde{A}_{0}\right)$ has only one parabolic face which lies in $\partial B_{\infty} \cap \gamma\left(X_{i}^{-}\right)$, which is an infinite Euclidean strip between two parallel Euclidean lines. Note that every frontier face of $\gamma\left(\tilde{A}_{0}\right)$ separates $\gamma\left(X_{i}^{-}\right)$. It follows that if $\gamma\left(\tilde{A}_{0}\right)$ has at least two parabolic faces in $\gamma\left(X_{i}^{-}\right) \cap \partial B_{\infty}$, then there must exist a frontier face of $\gamma\left(\tilde{A}_{0}\right)$ with two opposite sides contained in the strip $\gamma\left(X_{i}^{-}\right) \cap \partial B_{\infty}$ as essential arcs. But this contradicts Corollary 6.11, proving the claim.

Recall that we have assumed that every horoball component in $\mathcal{B}$, except $B_{\infty}$, has Euclidean diameter less than one. It follows that the Euclidean diameter of the set $\gamma\left(\tilde{A}_{0}\right) \backslash D_{0}$ is some fixed number independent of $n$. But the Euclidean diameter of $D_{0}$ must be very large if $n$ is very large. Thus the annulus $\gamma\left(\tilde{A}_{0}\right)$ cannot exist if $n$ is sufficiently large. The lemma follows.

We may assume that the number $N_{i}>n$ has been chosen big enough so that the surface $S$ has no disk components.

Now for the former requirement that for each compressing disk $D$ of $H, D \cap A$ has at least two components, it is sufficient to show that $\partial H \backslash A$ is incompressible in $H$ (since the genus of $H$ is obviously larger than one). We show:

Lemma 13.6 If either both of $n_{1}$ and $n_{2}$ are bigger than one or $\Delta$ is bigger than one, then $\partial H \backslash A$ is incompressible in $H$.

Proof We call $\partial \breve{Y}_{i}^{-} \backslash \partial_{p} \breve{Y}_{i}^{-}$the horizontal boundary of $\breve{Y}_{i}^{-}$. It has two components and is incompressible in $\breve{Y}_{i}^{-}$. The boundary of $\bar{U}^{-}$can be divided into three parts: the parabolic boundary $\partial_{p} \bar{U}^{-}$, the frontier of $\bar{U}^{-}$in $\breve{Y}^{-}$, and the rest which we call the horizontal boundary of $\bar{U}^{-}$(which we denote by $\partial_{h} \bar{U}^{-}$). Figure 27 illustrates $\partial_{p} \bar{U}^{-}$; in this figure, the frontier boundary meets $\partial_{p} \bar{U}^{-}$in straight segments, and the horizontal boundary meets $\partial_{p} \bar{U}^{-}$in curved arcs.

Claim The horizontal boundary of $\bar{U}^{-}$is incompressible in $\bar{U}^{-}$. 
Proof of Claim We just need to prove the claim for each component $\bar{U}_{k}^{-}$of $\bar{U}^{-}$. First note that the boundary of the $I$-bundle $\breve{g}_{i}\left(\left(A N_{\left(R, X_{i}\right)}\left(K_{i, k}\right)\right)^{-}\right)$can be naturally divided into parabolic, frontier and horizontal boundaries as well. Let $A_{i, k}$ be the frontier boundary of $\breve{g}_{i}\left(\left(A N_{\left(R, X_{i}\right)}\left(K_{i, k}\right)\right)^{-}\right)$, and let $S_{i, k}^{\prime}$ be the horizontal boundary of $\breve{g}_{i}\left(\left(A N_{\left(R, X_{i}\right)}\left(K_{i, k}\right)\right)^{-}\right)$. Note that $A_{1, k} \cup A_{2, k}$ is the frontier boundary of $\bar{U}_{k}^{-}$ and that $\partial_{h} \bar{U}_{k}^{-}=\partial \bar{U}_{k}^{-} \backslash\left(\partial_{p} \bar{U}_{k}^{-} \cup A_{1, k} \cup A_{2, k}\right)$. Obviously $S_{i, k}^{\prime}$ is incompressible in $\breve{g}_{i}\left(\left(A N_{\left(R, X_{i}\right)}\left(K_{i, k}\right)\right)^{-}\right)$. Since each component of $S_{i, k}^{\prime}$ separates $\bar{U}_{k}^{-}$and carries the fundamental group of $\bar{U}_{k}^{-}$, each component of $\partial \bar{U}_{k}^{-} \backslash\left(\partial_{p} \bar{U}_{k}^{-} \cup A_{1, k}\right)$ is parallel in $\bar{U}_{k}^{-}$ to a component of $S_{i, k}^{\prime}$ and thus is incompressible in $\bar{U}_{k}^{-}$. The components of $A_{2, k}$ are all annuli and strips, and the core curve of every annulus component of $A_{2, k}$ is essential in $\bar{U}_{k}^{-}$. Therefore, $\partial_{h} \bar{U}_{k}^{-}=\left(\partial \bar{U}_{k}^{-} \backslash\left(\partial_{p} \bar{U}_{k}^{-} \cup A_{1, k}\right)\right) \backslash A_{2, k}$ is incompressible in $\bar{U}_{k}^{-}$. The proof of the claim is finished.

Returning to the proof of Lemma 13.6, suppose that there is a compressing disk $D$ for $H$ which is disjoint from the annuli $A$. We may assume that $D$ is chosen to minimize the components of $D \cap \partial_{p} \bar{U}^{-}$.

If $D \cap \partial_{p} \bar{U}^{-}$is empty, then $D$ is contained in $\bar{U}^{-}$(it cannot be in $C_{0}(\alpha)$ since $\partial C_{0}(\alpha) \backslash \partial_{p} Y^{-}$is a set of disks), contradicting the claim. Thus $D \cap \partial_{p} \bar{U}^{-} \neq \varnothing$. Certainly we may assume that $D \cap \partial_{p} \bar{U}^{-}$has no circle components. Let $\sigma$ be an arc component of $D \cap \partial_{p} \bar{U}^{-}$which is outermost in $D$. The arc $\sigma$ divides $D$ into two disks; let $D_{0}$ be the one whose interior is disjoint from $\partial_{p} \bar{U}^{-}$. Let $\beta=\partial D_{0} \cap \partial D$. Then $\partial D_{0}=\sigma \cup \beta$. Let $D_{*}$ be the component of $\partial_{p} \bar{U}^{-}$which contains the arc $\sigma$.

Figure 27 shows the parabolic boundary of $Y^{-}$near $D_{*}$. A pair of parallel straight lines in the figure (including the dotted line segments) is a part of a pair of circles which bounds a component of the original parabolic boundary of $\breve{Y}_{i}^{-}$. There are two such components at $D_{*}$, one from $\partial_{p} \breve{Y}_{1}^{-}$and the other from $\partial_{p} \breve{Y}_{2}^{-}$. We call the components of their intersections with $\partial D_{*}$ corners of $D_{*}$. Alternately, the four corners are the intersection components of the annuli $A$ with $D_{*}$.

We claim that the endpoints of $\sigma$ cannot separate the four corners in $\partial D_{*}$, ie a case like that shown in Figure 27 (b) or (c) is impossible. Indeed, the endpoints of $\sigma$ are also the endpoints of the connected arc $\beta$ whose interior is disjoint from the parabolic boundary of $Y^{-}$and the annuli $A$. So if a case like Figure 27 (b) or (c) happens, then $\beta$ cannot be contained in $\partial C_{0}(\alpha)$. For otherwise the geometric intersection number $\Delta$ would be one and $n_{1}$ or $n_{2}$ would be equal to one. The arc $\beta$ cannot be contained in the horizontal boundary of $\bar{U}^{-}$either. For the endpoints of $\sigma$ lies in different components of the horizontal boundary of $\bar{U}^{-}$. 


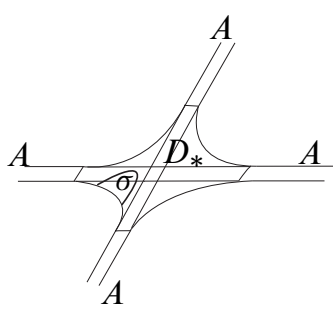

(a)

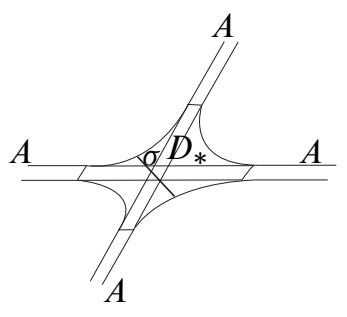

(b)

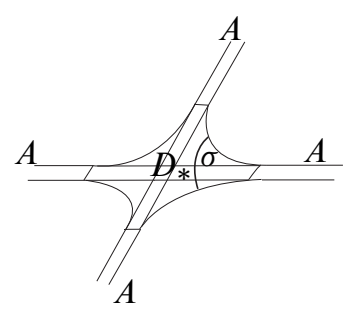

(c)

Figure 27

Hence $\sigma$ is contained in $D_{*}$ as shown in Figure 27 (a). Let $\beta^{\prime}$ be the sub-arc in $\partial D_{*}$ which is disjoint from the corners of $D_{*}$ and co-bounds a sub-disk $D_{1}$ in $D_{*}$ with $\sigma$. Then the union of $D_{0}$ and $D_{1}$ along $\sigma$ is a properly embedded disk in $H$ which we denote by $D_{2}$. Suppose that $\beta$ is contained in $\partial C_{0}(\alpha)$. Then $D_{0}$ is contained in $C_{0}(\alpha)$. Since $\partial C_{0}(\alpha) \backslash \partial_{p} Y^{-}$is a set of disks, $\partial D_{0}$ cannot be an essential curve in the torus $\partial C_{0}(\alpha)$. Thus $\partial D_{0}$ bounds a disk $D_{3}$ in $\partial C_{0}(\alpha)$. The two disks $D_{0}$ and $D_{3}$ form a 2 -sphere in $C_{0}(\alpha)$ and thus bound a 3-ball in $C_{0}(\alpha)$ (since $C_{0}(\alpha)$ is irreducible). Now it is clear that we can isotope the part of $D$ contained in the 3-ball to cross the sub-disk $D_{1}$ of $D_{*}$ and thus reduce the number of intersection components of $D \cap \partial_{p} \bar{U}^{-}$. Suppose then that $\beta$ is contained in the horizontal boundary of $\bar{U}^{-}$. Then $D_{0}$ is contained in $\bar{U}^{-}$and so is the disk $D_{2}$. Since the horizontal boundary is incompressible, $\partial D_{2}$ is not an essential curve in the horizontal boundary, ie $\partial D_{2}$ must bound a disk $D_{4}$ in the horizontal boundary. The two disks $D_{2}$ and $D_{4}$ form a 2-sphere in $\bar{U}^{-}$and thus bound a $3-$ ball in $\bar{U}^{-}$(since $\bar{U}^{-}$is irreducible). Again we can isotope the part of $D$ contained in the 3-ball to cross the sub-disk $D_{1}$ of $D_{*}$ and thus reduce the number of intersection components of $D \cap \partial_{p} \bar{U}^{-}$.

The proof of Proposition 13.4 is finished.

We now are in position to finish the proof of Theorem 1.1. Obviously $Y$ has non-empty boundary. Suppose $\Delta$ is bigger than one, or that both of $n_{1}$ and $n_{2}$ are bigger than one. Then we claim that $\left.f\right|_{\partial Y}$ is a quasi-Fuchsian surface. Indeed, since $f$ is injective on $\pi_{1} Y$, Part (1) of Proposition 13.4 implies that $f$ is injective in $\pi_{1} \partial Y$. Since $\partial Y$ is closed, then $\left.f\right|_{\partial Y}$ is not a virtual fiber. Therefore, by the Marden-Thurston-Bonahon classification of essential surfaces (see Section 1 Introduction), it is enough to show that $f^{*} \pi_{1} \partial Y$ contains no non-trivial parabolic elements.

The torus $\partial C_{0}$ is incompressible in $Y$ (otherwise $Y$ would be an open solid torus, which is obviously impossible). Hence $f^{*}\left(\pi_{1}\left(C_{0}, s_{1}\right)\right)$ is a finite index subgroup of the abelian group $\pi_{1}\left(\partial C, t_{1}\right)$. Hence if $\alpha$ is a non-trivial loop of $\partial Y$, and if $f \alpha$ is 
freely homotopic into $C$, then some non-zero power of $\alpha$ is freely homotopic into $C_{0}$, contradicting Proposition 13.4 Part (2).

Suppose then, that $\Delta=1$ and one of $n_{1}$ or $n_{2}$ (say $n_{1}$ ) is 1 . In this case we take the double cover of the manifold $Y$ dual to the non-separating surface $\breve{S}_{1}$ in $Y$ (note that $\breve{S}_{1}$ is naturally embedded in $Y$ ). Let $\hat{p}: \hat{Y} \rightarrow Y$ be the double cover. Then $\hat{Y}$ is a convex hyperbolic 3-manifold with a single cusp, which maps by a local isometry into $M$. Also $\hat{p}^{-1}\left(\breve{Y}_{1}^{-}\right)$has two components, and so in particular its parabolic boundary has two components on the boundary of the cusp $\hat{C}_{0}=\hat{p}^{-1}\left(C_{0}\right)$. Now we just need to show that every Dehn filling of $\hat{Y}$ gives a manifold whose boundary is incompressible. Let $\hat{Y}(\alpha)$ be any Dehn filling of $\hat{Y}$ along the cusp $\widehat{C}_{0}$ with slope $\alpha$. We give $\hat{Y}(\alpha)$ the obvious HS-manifold structure. Obviously the surface cross interval part of the HS-manifold has no simply connected components (since taking the double cover does not change this property). The rest of proof is exactly as that of Proposition 13.4 since the parabolic boundary of $\hat{p}^{-1}\left(\breve{Y}_{i}^{-}\right)$has at least two components now for each of $i=1,2$. This completes the proof of Theorem 1.1.

Acknowledgement The second author was partially supported by NSF grant DMS0204428 .

\section{References}

[1] C C Adams, Toroidally alternating knots and links, Topology 33 (1994) 353-369 MR1273788

[2] C C Adams, A W Reid, Quasi-Fuchsian surfaces in hyperbolic knot complements, J. Austral. Math. Soc. Ser. A 55 (1993) 116-131 MR1231698

[3] M Baker, D Cooper, A combination theorem for convex hyperbolic manifolds, with applications to surfaces in 3-manifolds arXiv:math.GT/0507004v3

[4] F Bonahon, Bouts des variétés hyperboliques de dimension 3, Ann. of Math. (2) 124 (1986) 71-158 MR847953

[5] R D Canary, D B A Epstein, P Green, Notes on notes of Thurston, from: "Analytical and geometric aspects of hyperbolic space (Coventry/Durham, 1984)", London Math. Soc. Lecture Note Ser. 111, Cambridge Univ. Press (1987) 3-92 MR903850

[6] D Cooper, D D Long, Virtually Haken Dehn-filling, J. Differential Geom. 52 (1999) 173-187 MR1743462

[7] D Cooper, D D Long, Some surface subgroups survive surgery, Geom. Topol. 5 (2001) 347-367 MR1825666

[8] D Cooper, D D Long, A W Reid, Essential closed surfaces in bounded 3-manifolds, J. Amer. Math. Soc. 10 (1997) 553-563 MR1431827 
[9] M Culler, P B Shalen, Bounded, separating, incompressible surfaces in knot manifolds, Invent. Math. 75 (1984) 537-545 MR735339

[10] D B A Epstein, A Marden, Convex hulls in hyperbolic space, a theorem of Sullivan, and measured pleated surfaces, from: "Analytical and geometric aspects of hyperbolic space (Coventry/Durham, 1984)", London Math. Soc. Lecture Note Ser. 111, Cambridge Univ. Press (1987) 113-253 MR903852

[11] I Kapovich, A Myasnikov, Stallings foldings and subgroups of free groups, J. Algebra 248 (2002) 608-668 MR1882114

[12] $\mathbf{T} \mathbf{L i}$, Immersed essential surfaces in hyperbolic 3-manifolds, Comm. Anal. Geom. 10 (2002) 275-290 MR1900752

[13] A Marden, The geometry of finitely generated kleinian groups, Ann. of Math. (2) 99 (1974) 383-462 MR0349992

[14] K Matsuzaki, M Taniguchi, Hyperbolic manifolds and Kleinian groups, Oxford Mathematical Monographs, The Clarendon Press, Oxford University Press, New York (1998) MR1638795

[15] W Menasco, Closed incompressible surfaces in alternating knot and link complements, Topology 23 (1984) 37-44 MR721450

[16] U Oertel, Closed incompressible surfaces in complements of star links, Pacific J. Math. 111 (1984) 209-230 MR732067

[17] J G Ratcliffe, Foundations of hyperbolic manifolds, Graduate Texts in Mathematics 149, Springer, New York (1994) MR1299730

[18] P Susskind, Kleinian groups with intersecting limit sets, J. Analyse Math. 52 (1989) 26-38 MR981494

[19] W P Thurston, The topology and geomety of 3-manifolds, Lecture notes, Princeton University (1979)

[20] Y-Q Wu, Immersed essential surfaces and Dehn surgery, Topology 43 (2004) 319-342 MR2052966

Mathematics Department, SUNY at Buffalo

Buffalo, NY 14290, USA

jdmaster@buffalo.edu, xinzhang@buffalo.edu

Proposed: Dave Gabai

Received: 28 March 2007

Seconded: Jean-Pierre Otal, Walter Neumann

Revised: 1 February 2008

Geometry 83 Topology, Volume 12 (2008) 